Volker Zimmermann

\title{
„Eine Medicinische Facultät in Flor bringen"
}

Zur Geschichte der Medizinischen Fakultät der Georg-August-Universität Göttingen

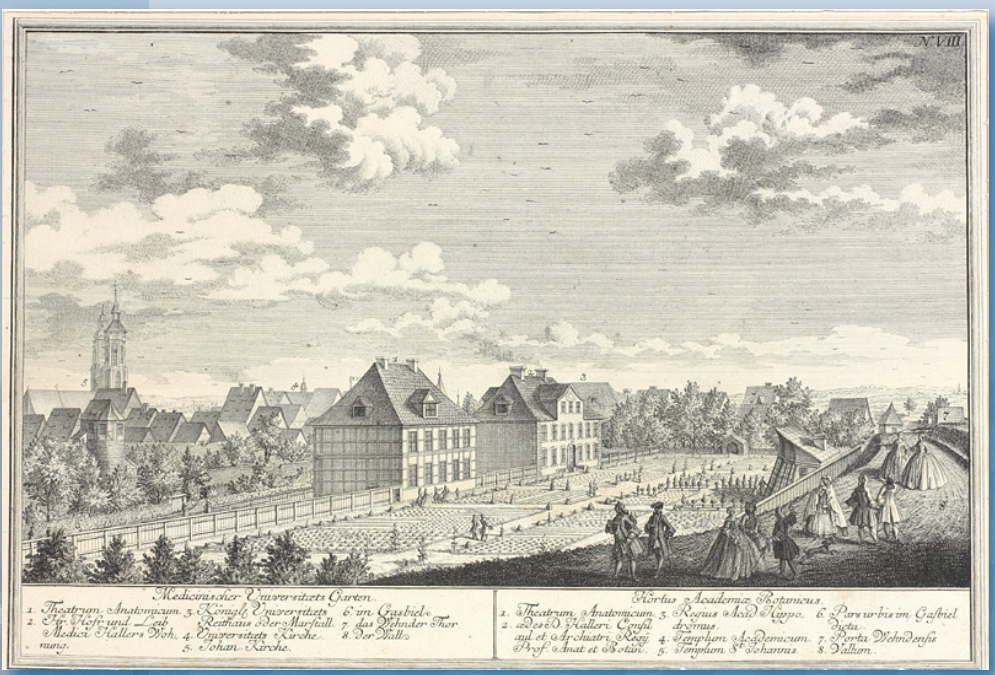

Göttingen. Anatomie.

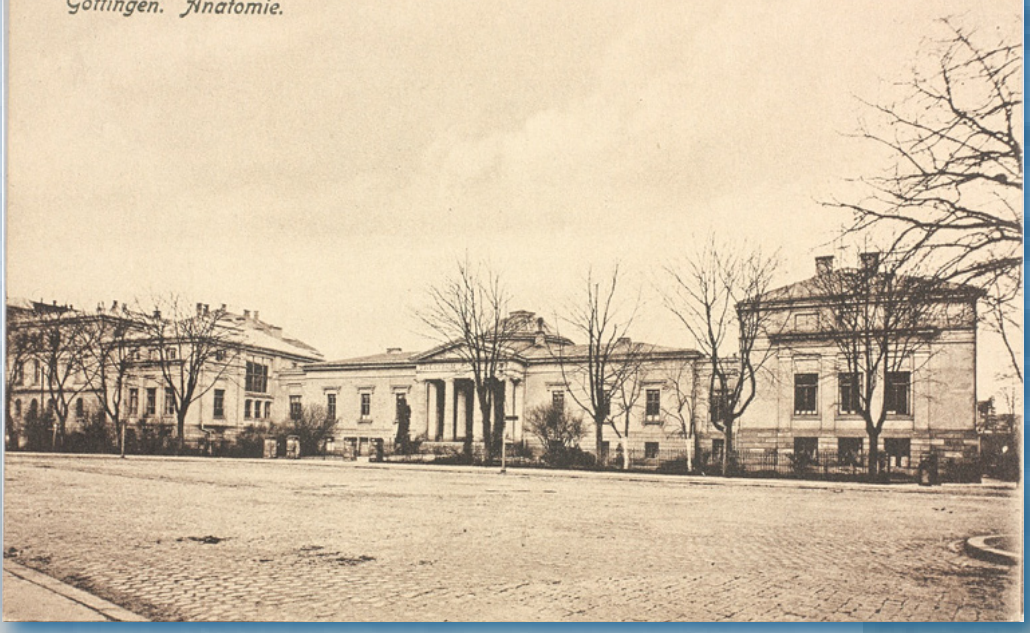



Volker Zimmermann

„Eine Medicinische Facultät in Flor bringen“

This work is licensed under the Creative Commons License 2.0 "by-nd", allowing you to download, distribute and print the document in a few copies for private or educational use, given that the document stays unchanged and the creator is mentioned. You are not allowed to sell copies of the free version.

SORIE RIGHIS RESERVED 
erschienen im Universitätsverlag Göttingen 2009 
Volker Zimmermann

„Eine Medicinische

Facultät in Flor bringen“"

Zur Geschichte der

Medizinischen Fakultät der

Georg-August-Universität

Göttingen

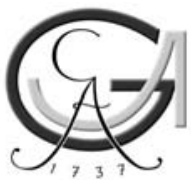

Universitätsverlag Göttingen

2009 


\section{Bibliographische Information der Deutschen Nationalbibliothek}

Die Deutsche Nationalbibliothek verzeichnet diese Publikation in der Deutschen Nationalbibliographie; detaillierte bibliographische Daten sind im Internet über $<$ http://dnb.ddb.de $>$ abrufbar.

Anschrift des Autors

Volker Zimmermann

e-mail: vzimmer@gwdg.de

www: http:/ /www.egm.med.uni-goettingen.de

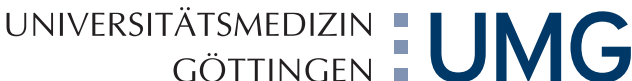

Die Publikation wurde durch die Universitätsmedizin Göttingen gefördert.

Dieses Buch ist auch als freie Onlineversion über die Homepage des Verlags sowie über den OPAC der Niedersächsischen Staats- und Universitätsbibliothek (http://www.sub.uni-goettingen.de) erreichbar und darf gelesen, heruntergeladen sowie als Privatkopie ausgedruckt werden. Es gelten die Lizenzbestimmungen der Onlineversion. Es ist nicht gestattet, Kopien oder gedruckte Fassungen der freien Onlineversion zu veräußern.

Satz und Layout: Anna Shemyakova Umschlaggestaltung: Margo Bargheer Titelabbildung:

Westansicht der Universitätsmedizin Göttingen. Aufnahme Stefan Rampfel Historische Göttinger Abbildungen

oben: Botanischer Garten an der unteren Karspüle mit Anatomie, Hallers Wohnhaus und Gewächshaus, Kupferstich von Georg Daniel Heumann, 1758;

unten: Anatomie neben dem Zoologischen Institut in der Berliner Straße, historische Postkarte

(C) 2009 Universitätsverlag Göttingen

http:/ / univerlag.uni-goettingen.de

ISBN: 978-3-940344-98-4 


\section{Inhaltsverzeichnis}

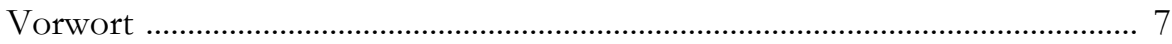

1. Göttingen - eine Universität für die Welt ....................................................... 9

2. Die Gründung der Medizinischen Fakultät .................................................. 11

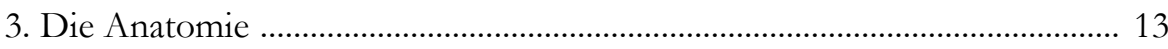

Die Anatomie im Turm am Albanitor ......................................................... 13

Das anatomische Theater am botanischen Garten .................................... 17

Der klassizistische Neubau außerhalb des Walls ......................................... 23

4. Über die Anfänge des praktisch-klinischen Unterrichts ............................... 29

Institutum clinicum regium ........................................................................ 30

5. Vom Gasthaus zum Großklinikum ……………………………………….... 33

Das akademische Hospital ............................................................................ 33

Himlys Hospital am Leinekanal ................................................................... 38

Enst-August-Hospital ......................................................................... 41

Die Kliniken an der Humboldtallee ..............................................................4

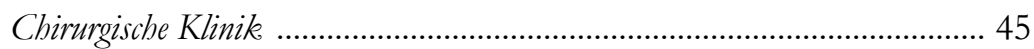

Medizinische Klinik ............................................................................. 47

Absonderungsbaracken ......................................................................... 48

Die Entwicklung der Frauenklinik.............................................................. 49

Armenhospital St. Crucis ....................................................................... 49

Interimslösung bei der Marienkirche .......................................................... 52

Accouchier-Hospital ………………………………………………….... 53

Frauenklinik an der Humboldtallee ....................................................... 58

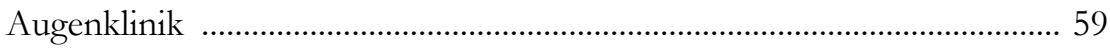

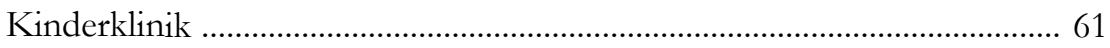

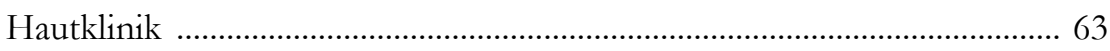

Die Hautklinik am Steinsgraben ............................................................. 64

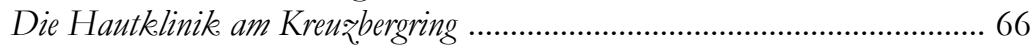

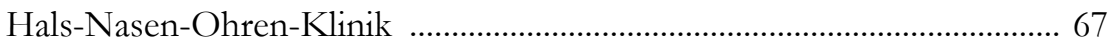

Die Entwicklung der Psychiatrischen Klinik .............................................. 70

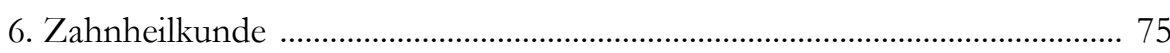


7. Die Pathologie und ihre Institutionalisierung ........................................... 81

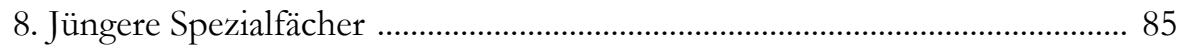

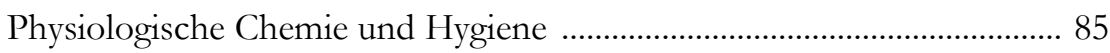

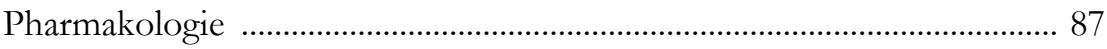

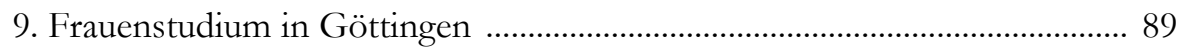

10. Die Medizinische Fakultät während der NS-Diktatur ............................. 93

Gleichschaltung und Entlassungspolitik ................................................... 93

Karl Saller und die Einrichtung eines „Lehrstubls für Rassenhygiene“ ......... 94

Zwangssterilisationen an Universitätskliniken .......................................... 95

Gottfried Ewald und die Auswirkungen der Euthanasie

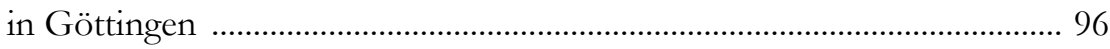

Der „Fall“"Hermann Rein ......................................................................... 97

Rudolf Stich - Dekan der Medizinischen Fakultät während des

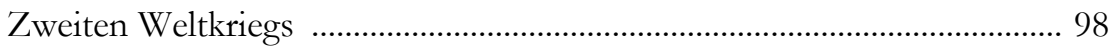

Zwangsarbeiter in den Universitätskliniken ............................................. 99

11. Sammlungen der Medizinischen Fakultät ................................................. 101

Die „Humanembryologische Dokumentationssammlung Blechschmidt" der Abteilung Embryologie des Zentrums Anatomie .................................... 101

„Symbole des Weiblichen“.

Die kulturgeschichtliche Sammlung Heinz Kirchhoff ............................ 102

„Metalla ferri. optumo pessimoque vitae instrumento“.

Die Sammlung zur Geschichte der Geburtshilfe .................................... 105

Die „Blumenbachsche Schädelsammlung“in der Göttinger Anatomie .......... 107

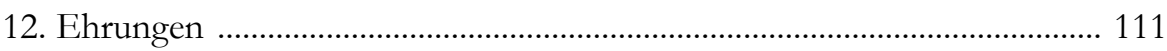

Die Albrecht von Haller-Medaille .......................................................... 111

Die Jacob Henle-Medaille ....................................................................... 114

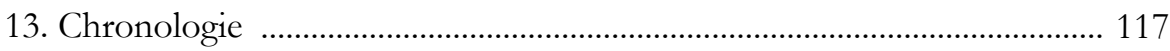

14. Zentren und Abteilungen der

Medizinischen Fakultät im Jahre 2009 ..................................................... 119

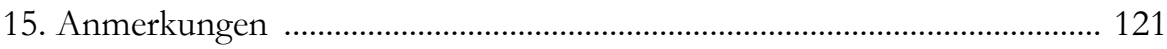

16. Quellen und Literatur ...................................................................... 131

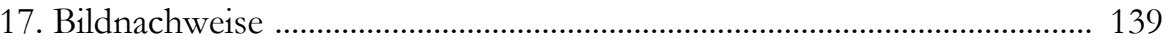




\section{Vorwort}

Die Gründung der Georg-August-Universität Göttingen beruhte auf einem weitgehend neuen Konzept, das vom Geist der Aufklärung durchzogen auf Toleranz gründete und jeglichem Extremismus und Sektierertum abhold war. Diesem Prinzip war auch die Medizinische Fakultät von Anfang an verpflichtet. Durch die Berufung entsprechender Professoren, an deren Spitze zweifelsohne Albrecht Haller stand, gelang es bereits in der Aufbauphase entscheidende Impulse zu setzen, die für die medizinische Ausbildung über Göttingen hinaus richtungsweisend wurden. Damit trug die Medizinische Fakultät wesentlich dazu bei, den internationalen Ruhm der Georgia Augusta zu begründen.

Diese historische Entwicklung will der vorliegende Abriss aufzeigen. Sein Schwerpunkt liegt naturgemäß auf den ersten beiden Jahrhunderten, da sich die Medizinische Fakultät in diesem Zeitraum in ihrer ganzen Breite entfaltete.

$\mathrm{Da}$ auf die praktisch-klinische Ausbildung am Krankenbett besonderer Wert gelegt wurde, kommt der jeweiligen Krankenhausentwicklung eine gewichtige Rolle zu, die für die einzelnen Spezialfächer entsprechend dokumentiert wird.

Die Geschichte der Zahnheilkunde zeigt ihren langwierigen Kampf um akademische Anerkennung und entsprechende Einbindung in die Medizinische Fakultät.

Die Entstehung und Institutionalisierung signifikanter Spezialfächer aufgrund neuer wissenschaftlicher Erkenntnisse spiegelt die Darstellung der „Pathologie“, der „Physiologische Chemie“, der „Hygiene" und der „Pharmakologie“ wider. Das Kapitel „Frauenstudium “ offenbart einen fakultätsinternen Mentalitätswandel.

Die gründliche Auseinandersetzung mit der eigenen Rolle während der NSDiktatur initiierte die Medizinische Fakultät durch die Öffentliche Ringvorlesung im Wintersemester 1989/90. Die wissenschaftliche Aufarbeitung wurde in der Folgezeit mehrfach finanziell unterstützt und als sichtbares Zeichen errichtete die Medizinische Fakultät 2008 einen Gedenkstein für die Zwangsarbeiter vor der alten Frauenklinik an der Humboldtallee.

Ihre Außendarstellung verbunden mit ihrem wissenschaftlichen Anspruch repräsentieren die Albrecht von Haller- und die Jacob Henle-Medaille und die dafür ausgewiesenen Preisträger. 
Das gesamte Panorama des Abrisses mit seiner vielschichtigen Realität gibt Einblicke und vermittelt Einsichten in das Selbstverständnis, die Selbstwahrnehmung und die Selbstdarstellung der Medizinischen Fakultät in ihrer Geschichte.

Mein Dank gilt zunächst dem Dekan der Medizinischen Fakultät Prof. Dr. Cornelius Frömmel, der die Arbeit anregte und ihr Entstehen großzügig unterstützte. Danken möchte ich den studentischen Hilfskräften Anna Shemyakova, die den gesamten technischen Teil mit bemerkenswerter Ruhe und Souveränität meisterte, Eva Shenia Shemyakova für ihre fachspezifischen Ratschläge und Alexander Weiß für Grafik und Lageplan. Frau Günther-Fecke und Frau Drost-Siemon vom Institut für Ethik und Geschichte der Medizin danke ich für die vielfältige Hilfe. Für anregende Diskussionen bleibe ich fortdauernd Bianca-Maria Zimmermann verpflichtet.

Göttingen, Mai 2009

Volker Zimmermann 


\section{Göttingen - eine Universität für die Welt}

Erste Entwürfe zur Gründung einer Universität in Göttingen sind aus der Mitte des Jahres 1732 belegt. Neben dynastischen Interessen, auf dem eigenen Territorium eine hohe Schule zu errichten, bot das aus der Reformationszeit stammende gymnasium illustre mit seinem exzellenten Ruf vorzügliche Bedingungen für die Gründung einer Landesuniversität. Die Verwirklichung lag in den Händen des Hannoverschen Geheimen Rats und späteren Premierministers Gerlach Adolph von Münchhausen (1688-1770). In seinem Auftrag erarbeitete der Hofrat Johann Daniel Gruber (1686-1748) erstmals drei Vorschläge, die sich im Wesentlichen mit der Finanzierungsfrage und - relativ weitgefasst - mit der möglichen Gestalt der zu gründenden Universität beschäftigten. Für Münchhausen selbst standen jedoch zunächst die geistigen und organisatorischen Voraussetzungen im Vordergrund. Gestützt auf mehr als ein Dutzend einschlägiger Gutachten wurde ein weitgehend neues Konzept entwickelt, das durchzogen vom Geist der Aufklärung auf Toleranz gründete und jeglichen extremen oder sektiererischen Positionen abhold war. „Gelehrte Monopolia müszen nicht gestattet, sondern jedem Profes\%. erlaubet werden, auch die zu seiner Profeszion nicht gebörige disciplinen zu dociren. " ${ }^{1}$ Eine solche Wissenschaftsauffassung war zukunftsweisend und ging in ihrer Modernität vor allem auch durch den Verzicht auf jegliche Theologen-Zensur deutlich über das Vorbild Halle hinaus. Das Modell Göttingen wurde so zum herausragenden Beispiel einer aufgeklärten Universität im 18. Jahrhundert.

Von der Stadt Göttingen wurde das ehemalige Dominikaner-Kloster (Pauliner Kirche) zur Verfügung gestellt und das darin residierende Gymnasium im April 1734 feierlich exauguriert. Im Dezember 1735 war das sich unmittelbar anschließende Kollegiengebäude mit einer Bibliothek, einem Konzilienraum, einer Sekretariatsstube, einem Karzer und einem Kollegsaal für jede Fakultät fertiggestellt. Die meisten Professoren hielten die Vorlesungen jedoch in ihren Wohnhäusern ab. Gleichzeitig wurde ein Reitstall für die körperliche Ertüchtigung erbaut. 
Am 14. Oktober 1734 hielt der Professor für Philosophie Samuel Christian Hollmann (1696-1787) in einem nach eigener Aussage ,ehedem vermutblichen Frucbtund Getreyde-Saal" 2 die erste Vorlesung an der neu gegründeten, freilich noch nicht inaugurierten Universität. Rechtliche Grundlage war das Reskript der Regierung vom 9. Oktober 1734 an Professor Georg Christian Gebauer (1690-1773), wonach er als Kommissar den Betrieb der Universität initiieren sollte. Im Wintersemester 1734/35 waren insgesamt 147 Studenten immatrikuliert. Die offizielle und feierliche Einweihung fand am 17. September 1737 statt. Bereits seit 1736 trägt die Universität - wenn auch nicht einheitlich - den Namen Georgia-Augusta als Reminiszenz an den Stifter, Kurfürst Georg August, der von 1727 bis 1760 regierte und als Georg II. König von Großbritannien war. 


\section{Die Gründung der Medizinischen Fakultät}

Während dieser außerordentlich erfolgreichen Gründungsphase stand die Medizin im Vergleich zur Theologischen, Juristischen und Philosophischen Fakultät zunächst etwas am Rande. Die Ursache dafür lag wohl im Gutachten des Hofrats von Meier. Dieser in Halle ausgebildete Jurist sah nahezu zwangsläufig in der Juristischen Fakultät die tragende Säule einer Universität. Der Medizin räumte er hingegen einen weit geringeren Stellenwert ein, da es seiner Meinung nach für eine große Provinz genüge, wenn man „zehn bis fünfzehn junge Würgengel creiert damit die Menschen methodice auf Kirchboff geliefert werden. " "Glücklicherweise blieb dieser Vorschlag ohne Resonanz. In dem Hannoveraner Mediziner Paul Gottlieb von Werlhof (1699-1767) hatte Münchhausen nämlich einen brillanten und äußerst kompetenten Ratgeber für alle Fragen und Probleme, die es im Zusammenhang mit der zu gründenden Medizinischen Fakultät zu lösen gab. Mit seinem fundierten und weitsichtigen Gutachten vom 16. Dezember 1733 schuf Werlhof eine hervorragende Grundlage für ein Fakultätsmodell, das hinsichtlich der Ausbildung, der Gelehrsamkeit und der Krankenversorgung richtungsweisend werden sollte: „W Wa eine medicinische Facultät in Flor bringen kan, bestehet vornehmlich in fünf Stücken: 1. der anatomie, 2. der botanic, 3. der chymie, 4. der theoria medica, 5. der praxi. Wo nicht für alle diese Stücke eine solche Anstalt gemacht wird, die in die Augen fällt, so ist kein sonderlich ungemeiner Zufluss von studiosis medicinae zu boffen. " ${ }^{4} \mathrm{Zu}$ jedem Fach erstellte er einen Abriss der zeitgenössischen Situation, skizzierte die notwendige technische und finanzielle Ausstattung, machte Angaben zur Ausbildung der Studenten und nannte herausragende Professoren, deren Berufung anzustreben wäre. Doch konnte keiner der vorgeschlagenen Kandidaten für Göttingen gewonnen werden.

Der erste Professor der Medizinischen Fakultät war der Anatom, Physiologe und Botaniker Johann Wilhelm Albrecht (1703-1736), der im November 1734 aus Erfurt berufen wurde. Albrecht hatte in Jena, Wittenberg, Straßburg und Paris Medizin studiert. 1728 wurde er in seiner Geburtsstadt Erfurt Landphysikus und 1730 außerordentlicher Professor an der Medizinischen Fakultät der Universität. Vor seiner Berufung nach Göttingen hatte er folgende drei Schriften publiziert: „Obseruationes anatomicae" (1730), „Tractatus de tempestate" (1731) und "Tractatus de effectibus musices in corpus animatum" (1734). Seine Göttinger Publikationen "Programmum de vitandis erroribus in doctrina medica" 
(1735), „Programmum de vitandis erroribus in medicina mechanica“ (1735), „De loco quodam Hippocratis male explicato" (1735) zeigen, ${ }^{5}$ dass er in der hippokratischen Tradition stand, daneben aber auch zeitbedingte iatromechanische Ansichten vertrat. 


\section{Die Anatomie}

In der zweiten Hälfte des Mittelalters gelang es der Medizin durch verstärkt vorgenommene Sektionen menschlicher Leichen die vor allem durch die galenische Tradition bedingte Erstarrung zu überwinden. Diese Entwicklung gipfelte schließlich im Erscheinen von Vesals „Fabrica“(1543), mit der im engeren Sinne die „medirinische Neureit" begann.

Die Anatomie, oft in Verbindung mit Fächern wie Botanik und Physiologie, war in der Regel das erste Lehrfach mit einem arbeitsfähigen Institut an einer neugegründeten Medizinischen Fakultät. So auch in Göttingen.

\section{Die Anatomie im Turm am Albanitor}

Erstes Domizil der Anatomie war im Turm am Albanitor. Weder die recht kümmerliche Örtlichkeit noch die Tätigkeit standen in der Göttinger Bevölkerung in hohem Ansehen. „Der erste Professor der Anatomie Albrecbt musste sich gefallen lassen, mit seinen Cadavern in einen alten dumpfigen Stadtthurm sich zu begeben. Selbst für Geld konnte er keinen bekommen, der ibm dazu nur W asser trug, " ",weil man Leute, die sich dazu brauchen liessen, fast als unebrlich ansahe, und Menschenschinder öffentlich nannte; ja, die Jungen auf den Gaßen, wenn der gute Albrecht von seiner, auf der Wehnder Straße liegenden Wobnung, nach dem Albaner Thor hinauf gehen mußte, Ihm wobl Selbst mit diesem Ehren:Nabmen verfolgten. "7

Am 12. Dezember 1735 erteilte Albrecht bei der Sektion zweier männlicher Leichen den ersten Unterricht an der Medizinischen Fakultät. Das Schicksal war ihm jedoch nicht hold; bereits nach gut einem Jahr starb Albrecht Anfang 1736 an Tuberkulose. „Die ganze Medicinische Facultät war mit Ihm also ausgestorben." 8 Einen nachhaltigen Eindruck konnte Albrecht in seiner knapp bemessenen Frist nicht hinterlassen. Nicht einmal ein Porträt von ihm ist bekannt.

Gelang es zunächst nicht, die im Werlhofschen Gutachten ins Auge gefassten Berufungen zu verwirklichen, so konnten 1736 mit Georg Gottlob Richter (16941773) und Albrecht Haller (1708-1777) gleich zwei Mediziner aus der bedeutenden Boerhaave-Schule für Göttingen gewonnen werden. 


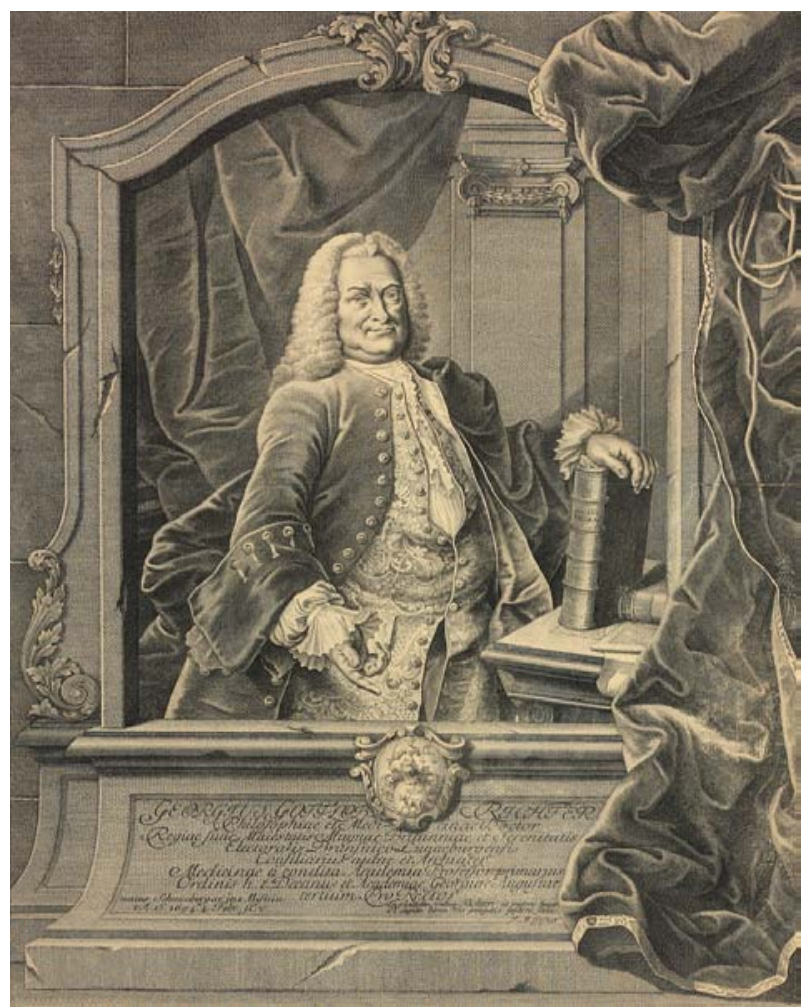

Abb. 1: Georg Gottlob Richter

Kupferstich von Georg Daniel Heumann, 1752

Richter, der sein Amt als Professor Medicinae primarius Ostern 1736 antrat, hatte in Leipzig, Wittenberg, Kiel und Leiden studiert. 1720 wurde er in Kiel zum Doktor der Medizin promoviert und hielt ,dasselbst Vorlesungen in medicis, philosophicis und litteris humanioribus. " Seit 1728 war er Leibarzt des Bischofs von Lübeck Adolf Friedrich von Hollstein-Gottdorf, dem späteren König von Schweden, in Eutin. Obwohl er als Boerhaave-Schüler mit dem „Leidener Modell“ und dessen stark praktischer Ausrichtung sicherlich vertraut gewesen war, praktizierte Richter einen herkömmlichen, weitgehend theoretischen Unterricht. So hielt er beispielsweise ein „collegium encyclopaedicum", ein „collegium diaeteticum“, ein "pathologicum collegium" und ein „collegium in materiam medicam“. Zwar las er auch ,über die praxin cum exercitiis clinicis“ 10 oder kündigte für das Sommersemester 1860 an: „Zur Praxi gibt Hr. Hofr. Richter eine Anweisung, da er geübten Zubörern medicinische Casus auszuarbeiten und zu beurtheilen vorlegt, " 11 doch entsprach der angekündigte Praxisbezug nach überlieferter Höreraussage nicht der Wirklichkeit. „In der Medicin ist er ein strenger Anbänger des großen Boerhave (sic!), allein ungeachtet seiner Erfahrung practicirt er überaus wenig und nur 
bey solchen Leuten, denen er entweder aus Freundschaft oder aus Hochachtung nicht abschlagen kann." 12 Sein wissenschaftliches Werk umfasst kleinere Schriften ,aber diese enthalten einen grossen Schatz klassischer Gelehrsamkeit, feiner kritischer und praktischer Bemerkungen über Stellen aus den Alten, und manche nütsliche praktische Wabrheit." 13 Insgesamt betrachtet erscheint das Wirken Georg Gottlob Richters innerhalb der Fakultät also eher blass, obwohl er als dienstältestes Mitglied nach der feierlichen Inauguration der Universität am 17. September 1737 der erste Dekan der Medizinischen Fakultät wurde. ${ }^{14}$

Von völlig gegenteiligem Naturell war Albrecht Haller (1708-1777), der im Herbst 1736 als zweiter Professor Medicinae ordinarius für Anatomie, Botanik und Chirurgie nach Göttingen kam. Wie kaum jemand nach ihm wurde er in den 17 Jahren seiner Göttinger Tätigkeit zur treibenden Kraft und gab der Universität und vor allem der Medizinischen Fakultät während der Aufbauphase entscheidende und richtungsweisende Impulse. „Es kann gar kein Zweifel sein, daß Albrecht Haller in erster Linie den internationalen Ruhm der Georgia Augusta begründet hat." 15

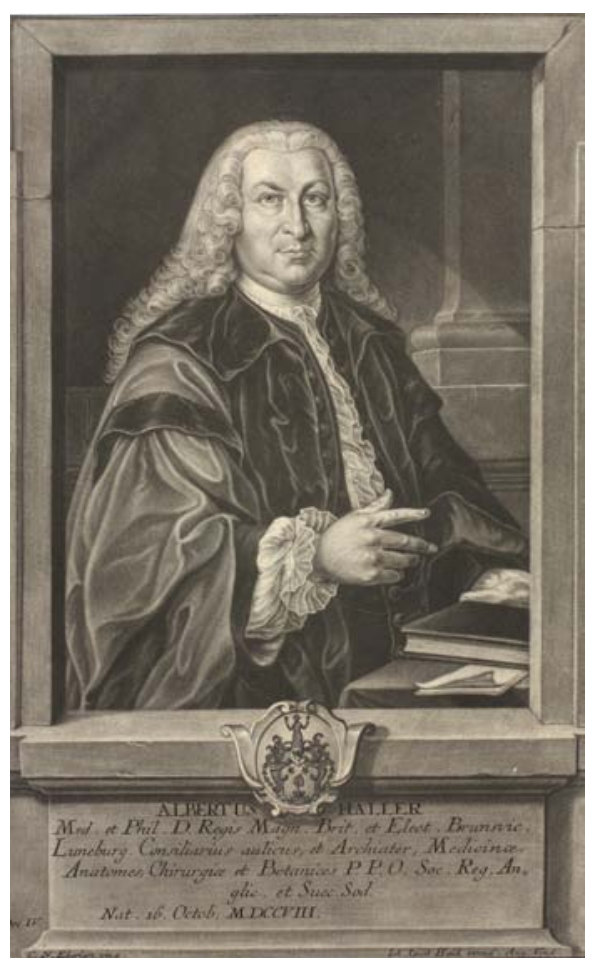

Abb. 2: Albrecht Haller

Schabkunstblatt, C. A. Eberlein pinx., Joh. Jacob Haid excud., o. J. 
Haller freilich initiierte den Ruf, der am 11. Mai 1736 an ihn erging, im Grunde selbst. Er hatte bei dem hannoverschen königlichen Leibarzt August Johann Hugo angefragt, ob an der neu gegründeten Göttinger Universität eine Stelle für ihn möglich wäre. Haller, der in Tübingen und Leiden, wo er 1726 zum Dr. med. promoviert wurde, studiert hatte, hatte bereits einige anatomische Arbeiten, beispielsweise über die Muskeln des Diaphragmas, über die Phthisis, die Peripneumonie und den Fötus sowie mehrere Schriften zur Botanik der Alpen veröffentlicht, ${ }^{16}$ und war als Verfasser des Gedichts „Die Alpen“ bekannt. Werlhoff, der in seinem einschlägigen Gutachten mehrfach auf das erfolgreiche „Boerbaave-Modell“ verwiesen hatte, und vor allem Münchhausen erkannten natürlich sofort, dass mit dem Boerhaave-Schüler Haller ihre anfänglichen Überlegungen zum Aufbau einer Medizinischen Fakultät nun verwirklicht werden konnten. Entsprechend groß war Münchhausens Bereitschaft, stets Hallers Wünsche zu erfüllen, und dieser nutzte die ihm entgegengebrachte Wertschätzung während seiner gesamten Göttinger Zeit jeweils unter Androhung seines Weggangs reichlich aus. ${ }^{17}$

Sein Jahresgehalt betrug anfänglich 640 Taler. Ferner erhielt er 400 Taler Umzugsbeihilfe und eine Zusage für zeitgemäße Arbeitsbedingungen. ${ }^{18}$ Hallers Ankunft in Göttingen am 30. September 1736 war jedoch überschattet. Bei der Einfahrt in die Stadt stürzte die Kutsche in einen Graben. An den Folgen dieses tragischen Unfalls verstarb seine Ehefrau vier Wochen später am 31. Oktober. ${ }^{19}$

Obwohl die äußeren Arbeitsbedingungen zunächst recht bescheiden waren, da sich Haller noch eine zeitlang mit dem Turm des Albanitors zufrieden geben musste, flüchtete er vollständig in seine Arbeit, wie sein Schüler und Biograph Johann Georg Zimmermann (1728-1795) in einer handschriftlich tradierten Aufzeichnung recht anschaulich berichtet: „Auf einem elenden alten Tburme in Göttingen nah an dem Albaner Thore suchte Haller die einzig mögliche Hülfe gegen seine Schwermuth. Dem Manne, dessen Augen an die schönste Natur gewöhnt waren, der die besten Gesellschaften gelebet hatte, blieb nun nichts mebr übrig als dieser Thurm der Vervü̈nschung. Alle Tage der Woche und sogar den sonst in Göttingen dem Umgange gevvidmeten Sonntag wühlte er in den menschlichen Eingewweiden herum, zog allen seinen Scharfsinn auf die Spitze seines anatomischen Messers zusammen und das Mittel half; zerstreut durch seinen Fleiss, mit neuen Wabrbeiten durch seinen edlen Eifer in allen Tiefen der Natur zu schauen." ${ }^{20}$ Von Anfang an also entzog sich Haller dem geselligen Leben. Sein insgesamt schwieriger Charakter entfremdete ihn zusehends den meisten seiner Kollegen, wenn er nicht sogar durch seine "leidenschaftliche Eigenheit" Verkehr und Umgang völlig abbrach. Wiederholt musste Münchhausen vermittelnd eingreifen. „Am meisten und fast allein verkehrte er mit dem frommen und treuen Theologen Oporin. " 21 


\section{Das anatomische Theater am botanischen Garten}

Für den 20. Juli 1737, also mitten im Sommer und entgegen bisheriger Gepflogenheit, kündigte Haller seine erste öffentliche Sektion an. Sie musste noch im Turm des Albanitores stattfinden, doch bereits im Mai des folgenden Jahres konnte die neu errichtete Anatomie am botanischen Garten, dessen Ausbau ebenfalls Hallers Werk war, bezogen werden. Daneben lag auch sein auf Staatskosten neu erbautes Wohnhaus, das er - ein sichtbares Zeichen Münchhausens, sich die Gunst Hallers beinahe um jeden Preis zu erhalten - kostenlos bewohnte. „Neben dem Theater wurde hiernächst demselben, an dem Garten, ein schönes Haus augebauet, mit der besondern Gnade=Bezeugung, daß er dasselbe umsonst empfieng, da sonst alle übrigen Professoren ibre empfangenen Häuser verbunden waren, jährlich zu verzinsen. Seine Pension wurd ibm gleich von Anfang her, ohne sein Begehren, ja obne sein Vorwissen, zu verschiedenen malen vermehret. Seine unverdrossenen Bemühungen in allem, was zur Aufnabme der Wissenschaften gereichen konnte, gab dem erlauchten Minister, dem die Besorgung der Universitet anvertrauet war, beständig neue Räthe an die Hand, die dessen sorgende Großmuth mehrentheils sogleich zum gemeinen Besten anwandte. "22 Haller selbst empfand dies als „une chose presque impossible. “

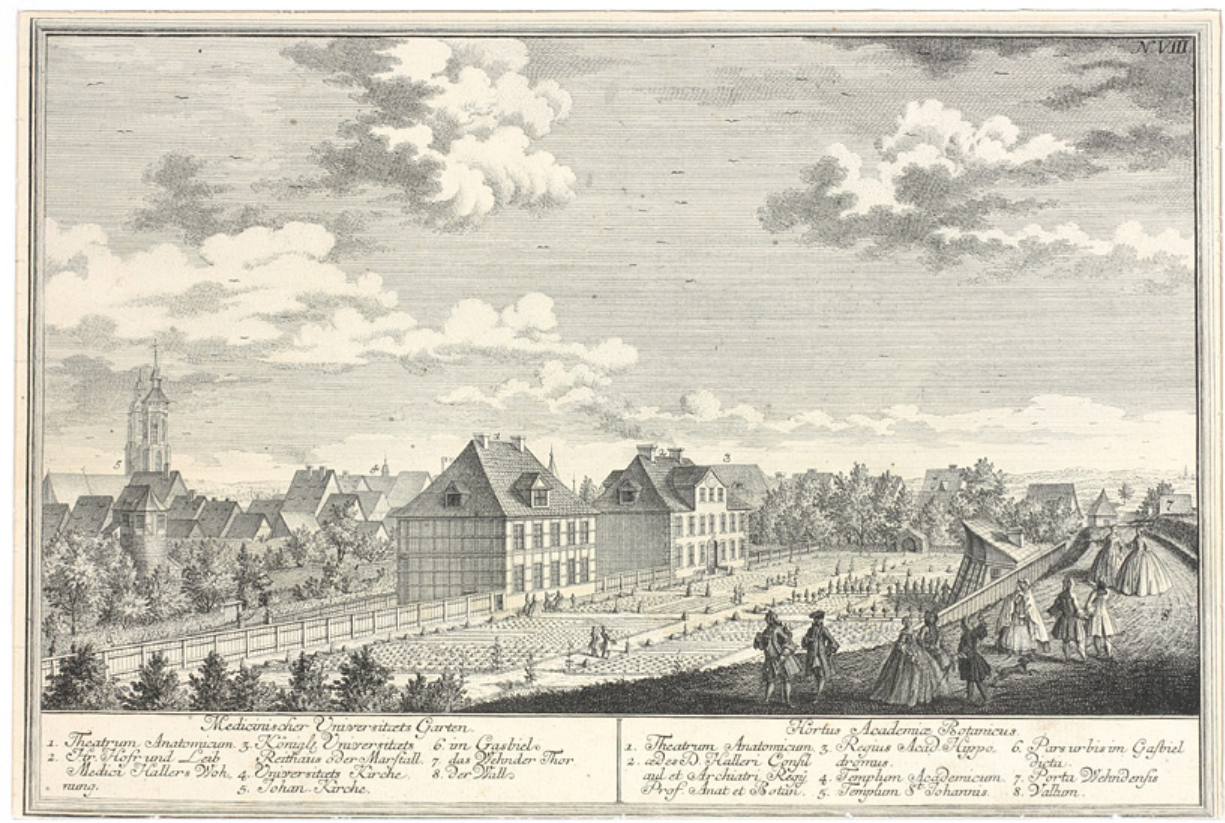

Abb. 3: Der Botanische Garten an der unteren Karspüle mit Anatomie, Hallers Wohnhaus und Gewächshaus Kupferstich von Georg Daniel Heumann, 1758 
Nach Hallers Vorstellungen entwarf der Klosterbaumeister Joseph Schädeler (1692-1763), der schon die Baumaßnahmen bei der Gründung der Universität geleitet hatte, eine Anatomie, die bis dahin einmalig in Deutschland war. Boerhaaves wissenschaftlicher Ansatz, die Ergebnisse der Naturwissenschaft für die Medizin verwertbar zu machen, wurde von Haller konsequent befolgt und in seinem neuen anatomischen Institut in die Tat umgesetzt.

Die Anatomie bestand aus insgesamt fünf Räumen, einem Hör- und Demonstrationssaal, einem Arbeitszimmer sowie einer Präparaten- und Injektionskammer. Der rechtwinklige Demonstrationssaal, in dessen Mitte ein nach allen Seiten hin beweglicher Demonstrationstisch stand, hatte sieben halbkreisförmige übereinander angeordnete Subsellien und bot ungefähr 200 Zuhörern Platz. Die Fenster waren so angelegt, dass das Licht ungehindert und ohne Schatten zu werfen einfallen konnte. ${ }^{23}$ Die Konstruktion war also durchdacht und die Ausstattung funktionsgerecht. Lediglich das Platzangebot erscheint in Anbetracht der durchschnittlichen Studentenanzahl von ungefähr 80 Medizinstudenten gegen Mitte des 18. Jahrhunderts ${ }^{24}$ als überdimensioniert. Doch wurde dabei sicherlich die in den Statuten der Medizinischen Fakultät von 1737 im \8 festgeschriebene Absicht berücksichtigt, zum einen Studenten anderer Disziplinen, die nach Kenntnissen des Körpers strebten, zu begeistern, zum anderen durch anatomische Demonstrationen eine breite Öffentlichkeit anzusprechen. ${ }^{25}$

Um eine solche Konzeption umsetzen zu können, musste auch für das entsprechende Unterrichts- und Forschungsmaterial gesorgt werden. Auf Grund des königlichen Privilegs vom 7. Dezember 1736 mussten die Leichen aller im Großraum Göttingen Hingerichteten kostenlos in die Anatomie gebracht werden. ${ }^{26}$ Dazu kamen alle tödlich Verunglückten, tot Aufgefundenen, durch Freitod umgekommene Menschen, die Leichen unehelicher Kinder oder deren Mütter, alle Armen, die kostenlos beerdigt werden mussten, die Leichen all jener, die ihren Körper nach dem Tod der Anatomie vermacht hatten, und schließlich alle im Hospital Verstorbenen. ${ }^{27}$ Haller hatte so in jedem Wintersemester 30 bis 40 Leichen zur Verfügung. Bei seinen Sektionen standen ihm ein akademisch gebildeter Prosektor, ein anatomischer Zeichner und zwei studentische Hilfskräfte zur Seite. ${ }^{28}$ Während seiner Göttinger Zeit präparierte er nach eigenen Angaben fast 350 Leichen selbst. ${ }^{29}$

Seinen anatomischen und physiologischen Unterricht sowie die Präparierkurse hielt er vormittags nach Vereinbarung ab; nachmittags widmete er sich den Demonstrationen und Übungen. Dabei beschränkte sich Haller nicht nur auf belehrende Demonstrationen, sondern bezog hervorragende Studenten durch ihnen übertragene eigenständige Aufgaben aktiv in seine Forschungsvorhaben mit ein. Durch diese kleineren Projekte, die sich in der Regel über zwei Wintersemester erstreckten, erhielt Haller zum einen eine nicht unwesentliche Unterstützung, zum anderen gereichte den beteiligten Kandidaten eine solche Mitarbeit nicht nur zur 
Ehre, sondern auch zur Vervollständigung ihrer anatomischen Kenntnisse. Diese seit 1746 praktizierte Unterrichtsmethode, die Lehre und Forschung verband und die er selbst als besten Weg zur Erkenntnistiefe ansah, ${ }^{30}$ wurde für die medizinische Ausbildung an den Universitäten richtungsweisend.

Im Sommersemester las er dagegen vorwiegend Chirurgie, Botanik, Physiologie, Semiotik und Gerichtsmedizin.

Während die Chirurgie in Hallers Denken eher eine untergeordnete Rolle spielte und er sie nie am lebenden Menschen ausübte, maß er seiner Aufgabe in der Botanik weit mehr Gewicht bei. Der Grund dafür dürfte in den Pariser Erfahrungen liegen, wo die Operationsergebnisse seines dortigen Lehrers HenriFrançois Le Dran (1685-1770) für ihn unbefriedigend waren. ${ }^{31}$ Sicherlich auf seine Anregung hin wurde neben der Anatomie mit erheblichen Mitteln ein vorzüglich ausgestatteter und weitläufig angelegter botanischer Garten 1739 fertiggestellt. Neben den üblichen Heilkräutern befanden sich auch spezielle, die Haller aus der Schweiz und dem Harz besorgt hatte, und wie sein einschlägiger Briefwechsel dokumentiert, stand er darüber hinaus in regem Pflanzenaustausch mit Spanien, Russland und China. ${ }^{32}$

Hallers schwermütiger Charakter, seine seelische Reizbarkeit und teilweise machtbesessene Selbstherrlichkeit führten innerhalb der Fakultät, seit Ende 1751 vor allem mit Brendel, immer wieder zu erheblichen Unstimmigkeiten, die Münchhausen, wenn auch nicht immer ohne Intrigen, durch materielle Zugeständnisse für beide Seiten, zu glätten verstand.

Johann Gottfried Brendel (1712-1758) hatte in Wittenberg studiert und wurde dort 1736 zum Dr. med. promoviert. 1738 kam er als Extraordinarius ohne bestimmtes Fachgebiet nach Göttingen und wurde 1739 zum Ordinarius befördert. ${ }^{33}$ In engerem Sinne war Brendel eigentlich nicht der Anatomie zugeordnet, doch war es gemäß \5 der Fakultätsstatuten jedem freigestellt, welches Fach der Heilkunde er vertreten wollte. Ebenfalls war dort festgelegt, dass zwei Professoren ihre Vorlesungen nicht zur gleichen Stunde ankündigen sollten, wobei die zeitliche und inhaltliche Koordination in den Händen des Dekans lag. ${ }^{34}$ Und gerade an dieser letzten Bestimmung, nicht an einer fachlichen Auseinandersetzung, entzündeten sich nach 13 Jahren die, äußerlich betrachtet, nichtigen Streitigkeiten.

Die eigentliche Ursache der Auseinandersetzung lag jedoch tiefer. Ende November 1751 verfügte die Regierung, dass jeder Mediziner, der sich im Königreich Hannover niederlassen wollte, an der Göttinger Anatomie noch vor der Promotion in zwei öffentlichen Demonstrationen seine anatomischen Kenntnisse nachzuweisen habe. Der jeweilige Professor der Anatomie musste ihm darüber kostenlos ein entsprechendes Zeugnis ausstellen. ${ }^{35}$ Es gibt wohl keinen Zweifel, dass Haller hinter dieser Anordnung steckte, die ihm einen weiteren Zuwachs seiner ohnehin großen Machtfülle in der Fakultät gebracht hätte. Aber die Medizinische Fakultät, deren Dekan Brendel war, leistete energischen Widerstand. Zwar wurde 
der Regierungserlass mit der Forderung nach zwei anatomischen Demonstrationen nicht abgelehnt, doch pochte man entschieden auf ein Mitspracherecht wie bei den anderen Examen. ${ }^{36}$ Zeitgleich und mit Wissen der Fakultät fixierte Haller selbst seinen Standpunkt. Mit Ausnahme der zeitlichen Festlegung der beiden anatomischen Demonstrationen, die entweder vor oder nach der Promotion stattfinden konnten, rechtfertigte er das gesamte vorgeschlagene Verfahren und verwies auf entsprechende Bestimmungen in Preußen. ${ }^{37}$

Ein weiteres Licht auf die extrem gespannte Situation in der Medizinischen Fakultät, deren Blockade Haller zutiefst getroffen hatte, wirft die Tatsache, dass er bereits einen Tag vor den beiden Stellungnahmen einen Beschwerdebrief an Münchhausen geschickt hatte, worin er das selbstherrliche und eigenmächtige Verhalten des Dekans Brendel aufs heftigste anprangerte. Er drohte unverhohlen mit seinem Weggang aus Göttingen und bat Münchhausen, vermittelnd einzugreifen, allerdings erst nach Brendels Amtszeit unter dem Dekanat Richters, das im Januar 1752 begann. ${ }^{38}$

Münchhausens Versöhnungsbemühen schien zunächst den gewünschten Erfolg zu erzielen, ${ }^{39}$ doch gegen Ende 1752 brach der Konflikt erneut aus, wobei es wiederum im Wesentlichen um die Festlegung der Vorlesungszeiten ging. Das erneute Einwirken auf Brendel, das von einer Rüge bis zur Gehaltserhöhung reichte, kam nun zu spät. ${ }^{40}$ Der Bruch war unausweichlich. Nach seiner letzten Promotion als Dekan am 17. März 1753 verließ Haller noch am Abend Göttingen. Vergeblich hatte Münchhausen bis zu seinem Tode 1770 gehofft, Haller zur Rückkehr nach Göttingen zu bewegen.

Als Präsident der Akademie der Wissenschaften, der er entscheidend zur Blüte verholfen hatte, und als eifriger Mitarbeiter an deren Publikationsorgan „Göttingische Gelehrte Anzeigen "blieb er allerdings zeitlebens mit Göttingen verbunden.

Hallers umfangreiches wissenschaftliches Werk spiegelt seine enormen Fähigkeiten wider, exakt zu experimentieren, gründlich zu beobachten und umfassend die einschlägige Literatur zu kennen. Göttingen bot ihm dazu die entsprechenden Arbeitsbedingungen. Aus seinem breit gefächerten anatomisch-physiologischen Forschungsgebiet sollen als pars pro toto die achtbändige „Elementa physiologicae corporis humani", die er selbst für die Krönung seines Lebenswerks hielt, ${ }^{41}$ und seine epochemachende Theorie der „Irritabilität" und „Sensibilität" genannt werden. Diese Theorie geht von der Überlegung aus, dass Fähigkeiten an Gewebestrukturen gebunden sind. Bei mechanischen, chemischen oder elektrischen Reizen zeigen die Organe mit Nervenfasern Schmerzempfindlichkeit (Sensibilität), die Organe mit Muskelfasern besitzen hingegen die Fähigkeit, sich zu verkürzen, ohne dabei Schmerz zu empfinden (Irritabilität). ${ }^{42}$ Hallers Lehre von der Irritabilität der Muskeln und der Sensibilität der Nerven schuf die Grundlage für die weitere Entwicklung der Physiologie und der allgemeinen Biologie. 
Nach Hallers Weggang verlor die Medizinische Fakultät ohne Zweifel an wissenschaftlichem Glanz. Alle Entscheidungen der unmittelbaren Folgezeit erweckten den Eindruck des Provisorischen, wohl weil immer noch mit Hallers Rückkehr gerechnet wurde. Ende 1753 rekrutierte Münchhausen zum Ordinarius der Anatomie Johann Georg Roederer (1726-1763), der seit 1751 als Extraordinarius die Gebärklinik leitete und dessen Neigungen eher der Geburtshilfe galten. ${ }^{43}$ Im gleichen Jahr wurde Johann Gottfried Zinn (1727-1759), ein Schüler Hallers, Extraordinarius und 1755 Ordinarius, starb aber bereits 1759. Brendel war im Jahr zuvor gestorben.

Nach Roederers überraschendem Tod auf einer Konsultationsreise nach Paris 1763 blieb die Professur für Anatomie drei Semester lang unbesetzt.

Ostern 1764 kam Philipp Georg Schröder (1729-1772) aus Marburg als Ordinarius für Medizin. Bereits nach dem Wintersemester 1764/65 gab er die Anatomie an Heinrich August Wrisberg (1736-1808), den einstigen Prosektor, ab, und las in der Folge über ausgewählte Texte der Alten, über besondere Therapie, Pathologie und Semiotik. Daneben hielt er klinische Übungen ab. ${ }^{44}$

Wrisberg hatte in Göttingen studiert, war 1764 promoviert und noch im gleichen Jahr mit viel Vorschusslorbeeren von der Fakultät zum Extraordinarius ernannt worden. ${ }^{45}$ Nach Schröders Rückzug wurde er als Professor der Anatomie eingesetzt. Gleichzeitig vertrat er bis 1766 die Chirurgie und bis 1785 die Geburtshilfe. Eine solche Fächerkombination war in den letzten Jahrzehnten des 18. Jahrhunderts an deutschen Universitäten durchaus die Regel. ${ }^{46} 1770$ wurde er zum Ordinarius ernannt. Während seiner insgesamt 44-jährigen Amtszeit sah er sich mehrfach und wohl nicht unbegründet Vorwürfen wegen schlechter Amtsführung ausgesetzt und musste sich bei der Regierung verantworten. ${ }^{47}$ Bei seinem Tod 1808 war der einstige Hallersche Glanz der Göttinger Anatomie weitgehend erloschen und die Medizinische Fakultät insgesamt in keiner guten Verfassung. „Die Verhältnisse waren schlechthin unerfreulich. Wrisberg war zwar endlich gestorben, er hatte viel getan, um den Charakter der Fakultät zu stören. " 48 Trotz des insgesamt schlechten Rufs hatte Wrisberg mehrere später bedeutend gewordene Schüler. Justus Christian Loder (1753-1832), der 1777 promoviert wurde und bereits 1778 als Ordinarius für Anatomie, Chirurgie und Geburtshilfe nach Jena berufen wurde, und Samuel Thomas Sömmering (1755-1830), der 1779 Lehrer der Anatomie und Chirurgie in Kassel am „Collegium Carolinum“wurde, zählten in ihrer Zeit zu den besten Anatomen Europas.

Johann Friedrich Blumenbach (1752-1840) trug nicht unwesentlich dazu bei, dem Ansehen der Medizinischen Fakultät wieder Geltung zu verleihen. Er wurde 1775 promoviert, 1776 Extraordinarius und 1778 Ordinarius. Mit seinem wissenschaftlichen Werk wurde Blumenbach zum Begründer einer auf anatomischphysiologischer Grundlage beruhenden Anthropologie, die auch ethnologischkulturelle Zusammenhänge einbezog. 


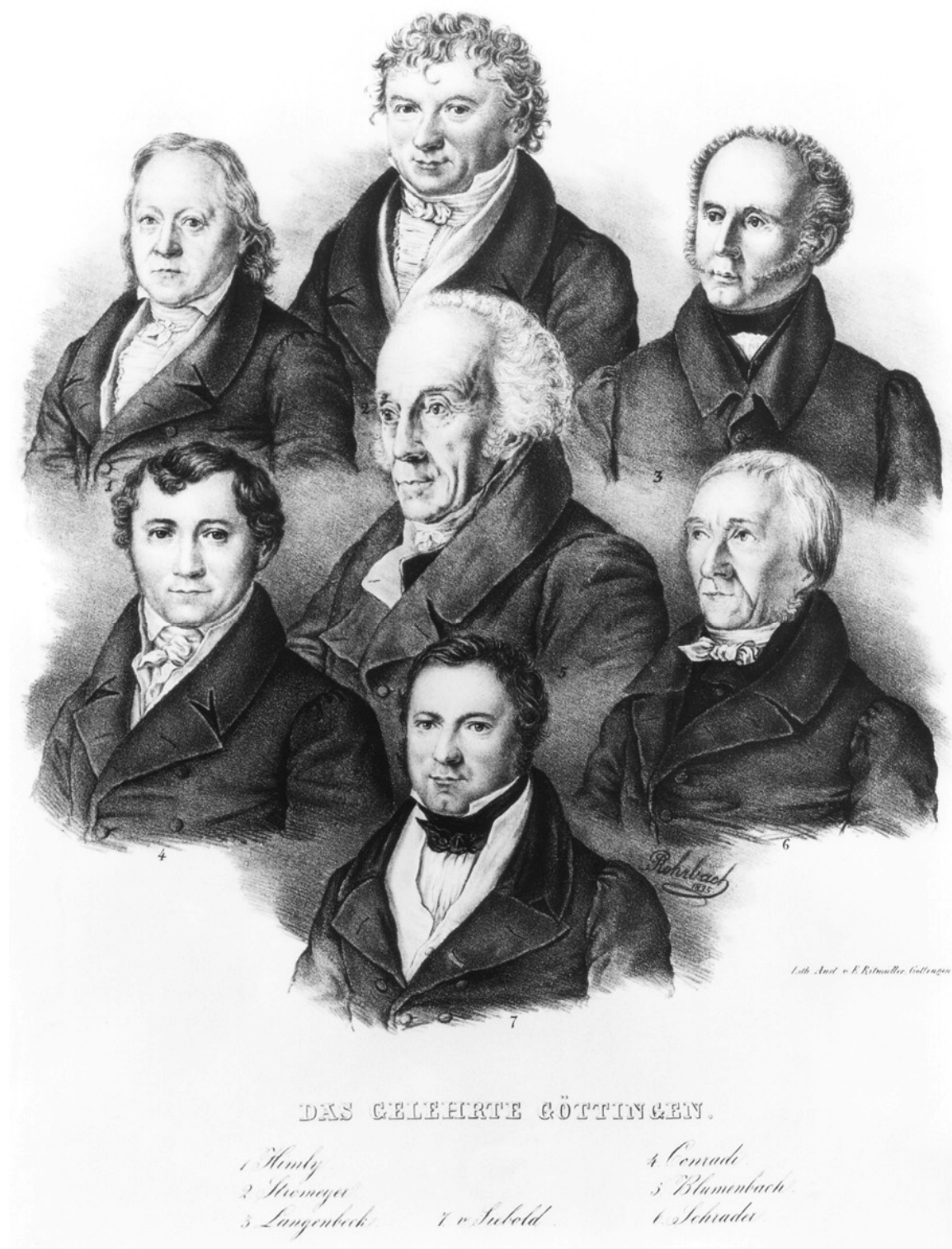

Abb. 4: Das gelehrte Göttingen

Lithographie von P. Rohrbach, 1835

Himly, Stromeyer, Langenbeck

Conradi, Blumenbach, Schrader

von Siebold 
Eine zentrale Rolle in seinem Denken nahmen die Überlegungen zum Bildungstrieb (,nisus formativus") ein. Danach besitzt jedes Lebewesen einen besonderen angeborenen und lebenslang wirksamen Trieb, der seine Entstehung initiiert und gestaltet, es am Leben erhält und bei einer Beschädigung wieder herstellt. Dieser Trieb, der zu den Lebenskräften gehört, ist von den übrigen Arten der Lebenskraft der organisierten Körper und von den allgemeinen physischen Kräften der Körper deutlich unterschieden. Er scheint, ohne dass die Ursache seiner Entstehung auszumachen ist, die erste wichtige Kraft zu aller Zeugung, Ernährung und Reproduktion zu sein. Präformierte Keime präexistieren also nicht. ${ }^{49}$ Auf der Grundlage seiner sorgfältig zusammengestellten Sammlung menschlicher Schädel entwickelte Blumenbach seine Theorie der Entfaltung der Menschheit in der Vielfalt der Rassen. ${ }^{50}$ Mit seinem wissenschaftlichen Werk trug er wesentlich dazu bei, die mechanistische Vorstellung von Lebewesen als Maschinen zu überwinden, und gewann dadurch nicht nur die Anerkennung Kants und Goethes. ${ }^{51}$

Bereits ab dem Sommersemester 1805 leitete Konrad Johann Martin Langenbeck (1776-1851), neben seiner klinischen Tätigkeit am akademischen Hospital, de facto die Anatomie. Er hatte in Jena studiert, dort 1798 den medizinischen Doktor gemacht und war seit 1802 Privatdozent der Anatomie und Chirurgie an der Medizinischen Fakultät in Göttingen. Angestellt als Wundarzt am akademischen Hospital wurde er 1804 Extraordinarius und 1814 schließlich Ordinarius. In seinem Bericht über das Wintersemester 1804/05 zählte er detailliert die Missstände und Versäumnisse auf. Mehrere Körper mussten demnach, ohne dass Präparationen vorgenommen waren, in teilweise bereits verwestem Zustand begraben werden. Lehrveranstaltungen vermittelten keine umfassenden Kenntnisse oder fanden überhaupt nicht statt. 52

\section{Der klassizistische Neubau außerhalb des Walls}

Auch unter Langenbecks Leitung erreichte die Anatomie nicht mehr den Glanz wie zu Zeiten Hallers, doch gelang ihm mit dem Neubau des „Theatrum anatomicum " eine deutliche Aufwertung. Höhere Studentenzahlen, immer umfangreicher werdende Präparatensammlungen und die aufgrund erweiterter Ausbildungsanforderungen verlangte höhere Anzahl von Leichen führten zu erheblichem Platzmangel, sodass ein Neubau zwingend erforderlich wurde. 1819 schlug Langenbeck der Regierung in Hannover einen Platz außerhalb des Walls in Verlängerung der Allee vor, der allen zeitgemäßen Erfordernissen entsprach. ${ }^{53}$ Die Regierung stimmte dem Bauvorhaben zu, ergriff aber zunächst keinerlei Initiative. Da sowohl die Unterrichtssituation als auch der bauliche Zustand immer unhaltbarer wurden, drängte Langenbeck 1826 deutlich entschiedener auf einen Neubau. Zur Finanzierung schlug er eine Anleihe bei privaten Geldgebern vor. ${ }^{54}$ Dies gelang. 
Am 18. April konnte mit dem Bau begonnen werden und am 2. November 1829 fand die feierliche Einweihung statt.

Die nach antikem Vorbild im klassizistischen Stil konstruierte Anatomie wirkte mit ihrem vorspringenden Säulenportal und den flügelartig zu beiden Seiten des kuppelartigen Amphitheaters angeordneten Räumen wie aus einem Guss und bildete recht eindrucksvoll eine harmonische Einheit zwischen Kunst und Wissenschaft. „Man schuf einen Tempel der Wissenschaft, dessen Altar der Seziertisch war. “ 55 Das zweistöckige Gebäude besaß im Erdgeschoss neben den spezifischen Funktionsräumen eine kleinere Wohnung für den Bediensteten und im Obergeschoss neben den Funktionsräumen auch drei Räume für die Sammlung. ${ }^{56}$

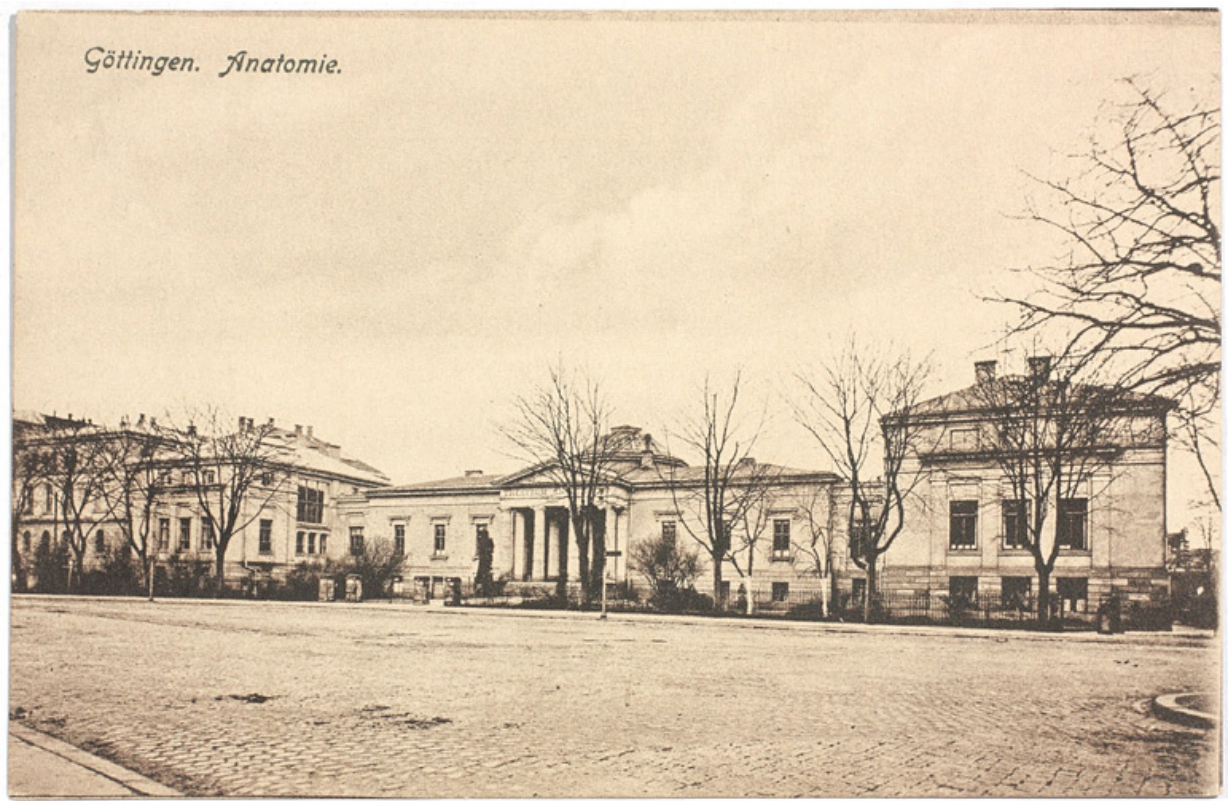

Abb. 5: Ansicht der „klassizistischen Anatomie“(1829-1945)

Historische Postkarte

(heute: Berliner Straße)

Städtebaulich passte sich die neue Anatomie als westlicher Abschluss einer auf sie zuführenden Allee gelungen ein. Von Anfang an weitsichtig geplant, diente dann der klassizistische Bau seinen Aufgaben, bis er am 7. April 1945 bei einem Fliegerangriff zerstört wurde. Nach Kriegsende fungierte die ehemalige Langenbecksche Chirurgie am Wilhelmsplatz als Notquartier, bis 1962 der Neubau der Anatomie am Kreuzbergring fertiggestellt war. 
War Albrecht Haller der absolute Glanzpunkt des 18. Jahrhunderts, so erreichte die Anatomie mit der Berufung Jacob Henles (1809-1885) ihren Höhepunkt im 19. Jahrhundert.

Es war die Zeit, in der einzelne Fächer nach Eigenständigkeit strebten. Zoologie und Chemie lösten sich vollständig aus der Umklammerung der Medizin, obwohl Friedrich Wöhler (1800-1882) bis zu seinem Tode Ordinarius an der Medizinischen Fakultät blieb. Rudolf Wagner (1805-1864), der seit 1840 als Nachfolger Blumenbachs Ordinarius für Physiologie, vergleichende Anatomie und Zoologie war, wollte Göttingen wie zu Hallers Zeiten zu einer Pflanzstätte der Physiologie machen und erhielt bereits 1842 ein selbstständiges Physiologisches Institut. Leider fehlte es ihm an Konsequenz und Stetigkeit. Als 1851 Langenbeck starb, übernahm er wieder die Anatomie; sein Versuch endete jedoch in einem Fiasko. Er zog sich daraufhin zurück und auch die Physiologie erfuhr durch ihn keine weitere Förderung mehr. Sein Nachfolger Georg Meissner (1829-1905) wurde 1860 erster Ordinarius des neuen Spezialfaches Physiologie, richtete im folgenden Jahr ein neues Laboratorium ein und konnte 1886 ein eigenes Institut beziehen. ${ }^{57}$

Da Langenbeck ab 1849 seine Tätigkeit auf die Anatomie beschränken musste, erhielt die Chirurgie eine gesonderte Professur, die Wilhelm Baum (1799-1883) übertragen wurde, und mit dem Amtsantritt Henles war nun auch die Anatomie ein eigenständiges Spezialfach.

Jacob Henle, der in Bonn, Heidelberg und Berlin studiert hatte, war Schüler von Johannes Müller (1801-1858). Nach Promotion (1832) in Bonn und Habilitation (1837) in Berlin vertrat er seit 1840 die Anatomie in Zürich und ab 1844 die in Heidelberg. Als er 1852 nach Göttingen kam, war er wohl einer der bedeutendsten deutschen Anatomen des 19. Jahrhunderts. ${ }^{58}$

Die noch verhältnismäßig neue und in allen Belangen funktionsgerecht ausgestattete Anatomie bot ihm eine hervorragende Arbeitsstätte, die er trotz eines Rufes nach Berlin (1858) nicht mehr verließ. Als genauer Beobachter und scharfsinniger Analytiker beeinflusste Henle durch seine streng mikroskopische Methode die anatomische Forschung grundlegend.

Seine „Pathologischen Untersuchungen“ enthalten vier Aufsätze, die noch in der Berliner Zeit konzipiert wurden. Im ersten „Von den Miasmen und Contagien und von den miasmatisch-contagiösen Krankheiten" sucht er nach deren Ursachen. Da Miasma und Kontagium jeweils die gleiche Krankheit hervorrufen, müssen sie identisch sein. Sie besitzen ferner Eigenschaften, die nur mit individuellem Leben ausgestattete Stoffe haben. Er folgerte, dass die Ansteckungsmaterie daher aus Lebewesen bestehen muss. ${ }^{59}$ Obwohl die Beweisführung logisch zwingend war, blieb die verdiente Anerkennung versagt, weil Henle die angenommenen Lebewesen nicht nachweisen konnte. Doch öffnete er mit seinen rein theoretischen Überlegungen den Weg zur Erforschung und erfolgreichen Bekämpfung der Seuchen und Infek- 
tionskrankheiten, die nicht zuletzt durch die bahnbrechenden Arbeiten von Henles Schüler Robert Koch (1843-1910) der Medizin des 19. Jahrhunderts gelang.

In seiner "Allgemeinen Anatomie“ erstellte Henle ein Klassifikationssystem, das in der ersten Abteilung die tierischen Elementarteile im Allgemeinen und in der zweiten die Gewebe strukturiert. ${ }^{60}$ Obwohl die morphologische Einteilung des Stoffes - auch in den Augen Henles selbst - sicherlich noch unvollkommen war, stellte sie doch einen deutlichen Fortschritt im Vergleich zu den herkömmlichen Systemen dar und verlieh der weiteren Entwicklung der Histologie entscheidende Impulse. Auch zahlreiche neue Erkenntnisse wurden in diesem Zusammenhang publiziert, es sei nur auf die Darstellung der Hornhaut und die hier erstmals exakt beschriebene glatte Muskulatur der Blutgefäße verwiesen. ${ }^{61}$

Das zweibändige „Handbuch der rationellen Pathologie" untersucht im allgemeinen Teil Begriff und Wesen der Krankheit, die allgemeine Ätiologie sowie die räumlichen und zeitlichen Beziehungen der Krankheit. Der spezielle Teil behandelt die Pathogenie, die Symptomatologie und die Ätiologie. ${ }^{62} \mathrm{Im}$ Wesentlichen geht es bei diesem Werk um Fragen, die die Medizin jener Zeit bewegten. Die raschen Fortschritte der Wissenschaft führten letztlich dazu, „dass das Buch schon nach kurzer Zeit aus dem Centrum der literarischen Besprechung herausrückte und vergessen wurde. "63
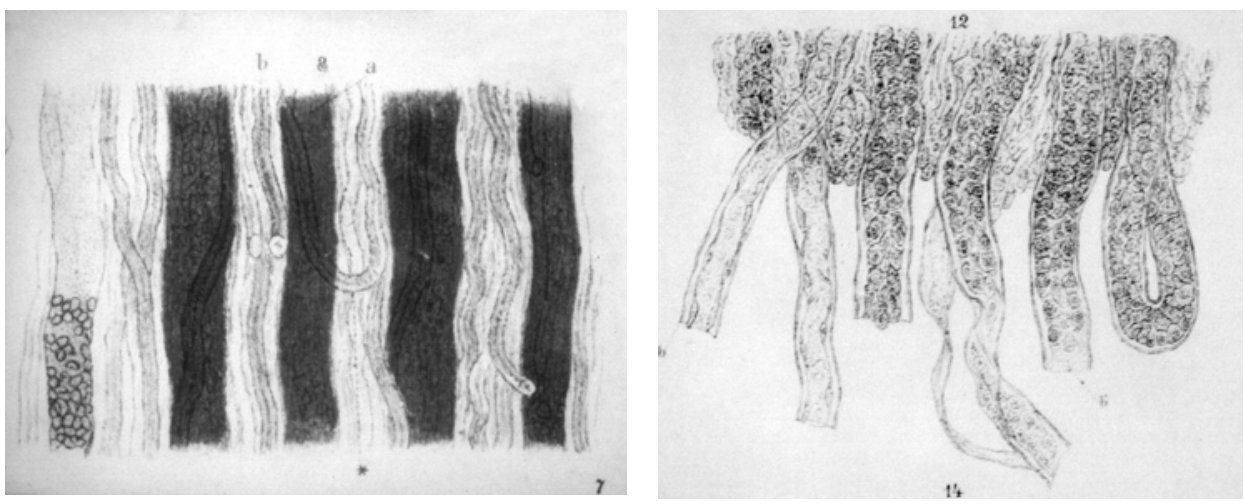

Abb. 6: Henlesche Schleife

Reproduktion aus der Erstveröffentlichung: „Zur Anatomie der Niere“, 1862

Die größte bleibende wissenschaftliche Leistung Henles war zweifelsohne seine Entdeckung der u-förmig gewundenen Abschnitte der Nierenkanälchen in der Nierenrinde, die die Voraussetzung für das Gegenstromprinzip schaffen. Über die Existenz der dann nach ihm benannten „Henleschen Schleife “berichtete er 1862 vor der Königlichen Gesellschaft zu Göttingen erstmals der Öffentlichkeit. ${ }^{64}$

Gleichsam als Vermächtnis seines gesamten wissenschaftlichen Schaffens muss das „Handbuch der systematischen Anatomie des Menschen" gesehen werden. In 
diesem dreibändigen „opus magnum“, das in einem Zeitraum von 24 Jahren erschien, entfaltete Henle sein reichhaltiges Wissen aus dem gesamten Spektrum seiner vergleichend anatomischen, histologischen oder physiologischen Forschungsschwerpunkte. Es war Henles Absicht, dem Werk durch eine gelungene Verbindung von Text und Abbildung, eine praktische Tendenz zu geben und so die Anregungen im Text durch entsprechende Abbildungen zu vertiefen. ${ }^{65}$ Die Wirkung war durchschlagend. „Das Buch reformirte die Anatomie und deren Darstellung so sehr, dass kein später erschienenes Hand- oder Lehrbuch der Disciplin sich seinem Einfluss, sei es absichtlich, sei es unabsichtlich, zu entziehen vermochte. " 66

Mit Albrecht Haller und Jacob Henle besaß die Anatomie und mit ihr die gesamte Medizinische Fakultät ihre bedeutendsten Köpfe in der bisherigen Geschichte. Bis heute steht die Medizinische Fakultät in deren Tradition. Dies zeigt sich auch daran, dass die beiden Medaillen, die die Medizinische Fakultät heute für herausragende wissenschaftliche Leistungen verleiht, deren Namen tragen. 



\section{4. Über die Anfänge des praktisch-klinischen Unterrichts}

Die in der Leidener Schule von Boerhaave konzipierte praktisch-klinische Unterrichtsmethode, zu der auch die vierstufige Krankenhausuntersuchung: ausführliche Anamnese, Feststellung des Status praesens, Besprechung der Diagnose und Prognose sowie die Bestimmung der Therapie zählte, fand auch in Göttingen ihren Niederschlag.

Den ersten wirklich praktisch-klinischen Unterricht gab seit 1755 Johann Gottfried Brendel. Die entsprechende Ankündigung lautete: „Zur practischen Medicin gebört das Clinicum des Hr. Prof. Brendel. "Auch unter der Bezeichnung „Practicum“ wird es drei Semester hindurch angekündigt. ${ }^{67}$ Brendel ,nahm seinen Schoosjünger mit an das Krankenbette" 68 und besprach anschließend die einzelnen Krankheitsfälle. $\mathrm{Da}$ er in und um Göttingen eine erfolgreiche Praxis betrieb, verfügte er über eine große praktische Erfahrung und über genügend Patienten zur Demonstration. Die Resonanz auf seinen Unterricht war dementsprechend positiv. „Sein Collegium practicum wird überaus hoch gehalten und ein Manuscript für 50 Thlr. bezablt... " 69

Im Wintersemester 1756/57 kündigte Rudolf Augustin Vogel (1724-1774) erstmals ein "practicum clinicum " an, das im folgenden Semester "öffentliches Clinicum" hieß. ${ }^{70}$ Dieses Klinikum fand mittwochs und sonnabends jeweils eine Stunde lang statt. Die Patienten waren arme Kranke aus der Stadt und den umliegenden Gebieten. Sie wurden in Gegenwart der Studenten untersucht; anschließend wurden die Krankheitsfälle besprochen und entsprechende Arzneien verordnet. Die bettlägerigen Stadtkranken wurden von Vogel selbst oder den Kandidaten besucht, die dann über ihre behandelten Fälle berichten und Tagebuch führen mussten. $\mathrm{Zu}$ wichtigen und interessanten Landpatienten wurde am Sonnabend gereist. Der Unterricht war für die Studenten nicht kostenlos; für die Bezahlung der Arzneien mussten sie vierteljährlich ,etwas gewisses "beitragen. Chirurgische Eingriffe besorgte der sonst nicht näher bekannte Universitäts-Chirurgus Tolle. ${ }^{71}$

Vogels praktisch-klinischer Unterricht war erfolgreich, wie aus einem studentischen Bericht, der hinsichtlich der Uroskopie allerdings Unkenntnis verrät, zu entnehmen ist. ,....seine Praxis scheinet zuzunehmen, zumabl da er sich nach der Einfalt der Leute richtet und noch den Urin besiehet, welches sonst kein biesiger Practicus thut. " 72

Der Letzte, der praktischen medizinischen Unterricht vor der Gründung des „Institutum Clinicum“ erteilte, war Philipp Georg Schröder (1729-1772). Von 1767 
bis zu seinem Tode führte er durchgehend ein „Collegium Clinicum" durch. Sein Unterricht war für die Studenten kostenlos; lediglich für die Arzneimittel, die den Armen verschrieben wurden, mussten sie aufkommen. ${ }^{73}$

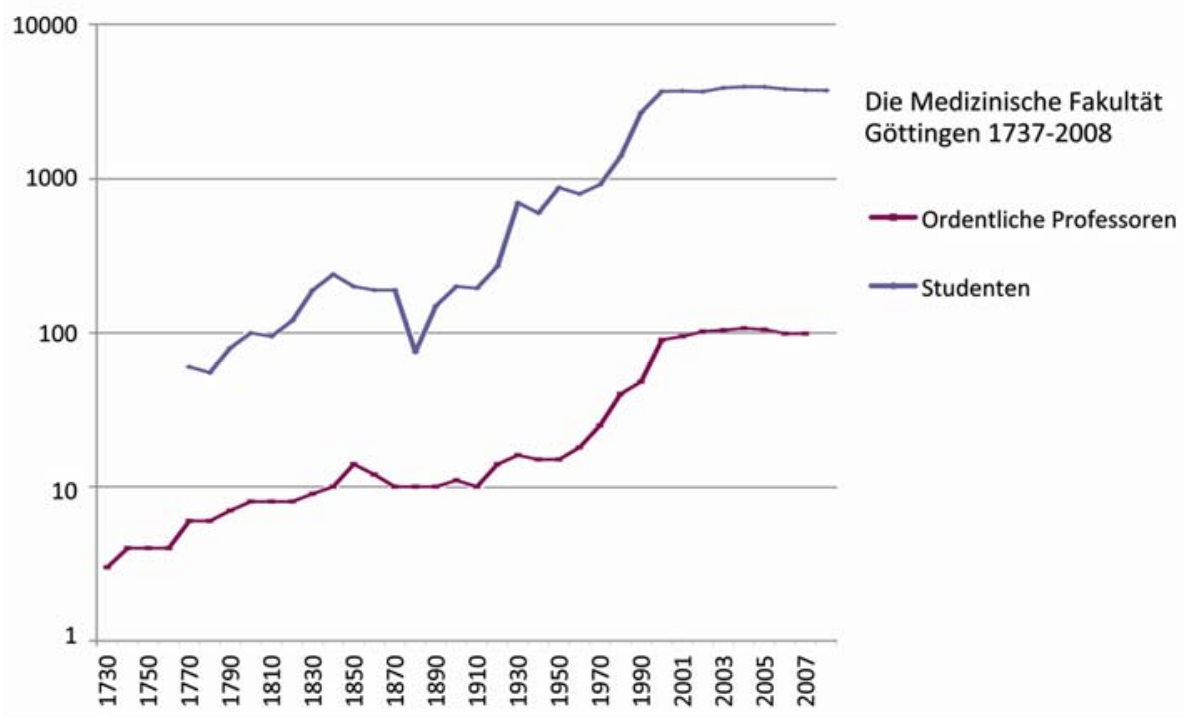

Abb. 7: Die Entwicklung der Medizinischen Fakultät

\section{Institutum clinicum regium}

Schröders Nachfolger wurde Ernst Gottfried Baldinger (1738-1804). Er hatte in Erfurt, Jena und Halle studiert, wo er 1760 promoviert wurde. 1768 wurde er Professor für Medizin in Jena und 1773 in Göttingen. Sofort nach seinem Dienstantritt richtete er am 17. Mai 1773 das „Collegium clinicum" ein. Da die Patientenzahl ständig wuchs, ersuchte er noch im Sommer desselben Jahres das Ministerium um einen jährlichen Zuschuss für die Arzneikosten von 200 Talern, der ihm auch gewährt wurde. Die Studenten mussten von nun an monatlich eineinhalb Taler zu den Unterhaltskosten des Klinikums beitragen. Baldinger selbst verzichtete auf eine Vergütung seiner Tätigkeit. Durch die von nun an regelmäßige staatliche Förderung war Baldingers Klinikum zu einer festen Einrichtung geworden und gleichsam in den Rang eines "Institutum clinicum regium" erhoben. In seiner Funktion war es somit nicht nur ein Laboratorium, sondern eine wirkliche Poliklinik. ${ }^{74}$ Das Klinikum, das wahrscheinlich in Baldingers Privathaus an der Ecke Gotmar- und Paulinerstraße stattfand, war von Montag bis Freitag jeweils zwischen 13 und $14 \mathrm{Uhr}$ festgesetzt. 
Neben der Möglichkeit, Kranke zu untersuchen, wurde auch eine „Allgemeine Lehre zur Praxis mit besonderer Anwendung auf die morbos pauperum Gottingensium "geboten. Die Studenten mussten darüber hinaus Aufsätze über praktische Themen schreiben und Rezepte verfassen, die von Baldinger dann korrigiert wurden. In den ersten Jahren kamen durchschnittlich 15 Hörer pro Semester - das waren $22 \%$ der Göttinger Medizinstudenten. Im Durchschnitt dauerte die Ausbildung fünf Monate. ${ }^{75}$

Baldinger verstand seinen klinischen Unterricht als Erziehung zur Selbstständigkeit. „Ich führte meine Zöglinge zum Selbsthandeln, ließ sie examiniren, consultiren, receptiren und führte nur das Steuerruder. "76 Baldinger selbst war großzügig und hilfsbereit und ließ stets einige arme Studenten ,gratis auscultando" an seinem Klinikum teilnehmen. Durch das 1781 eröffnete akademische Hospital, in dem nun ebenfalls praktischer Unterricht abgehalten wurde, erhielt das Baldingersche Klinikum erhebliche Konkurrenz. Das Klinikum wurde nur noch von 15\% der Medizinstudenten besucht.

Uneins mit seinen Kollegen, vor allem mit dem Leiter des akademischen Hospitals, August Gottlieb Richter, verließ Baldinger 1782 Göttingen und folgte einem Ruf des Landgrafen zu Hessen - Kassel, wo er Hofrat, erster Leibarzt und Professor der praktischen Medizin wurde. Damit war auch die Blütezeit des „Collegii clinici" zu Göttingen vorüber.

Ein zehnjähriges Interim mit wechselnden Leitern, unter denen auch Johann Peter Frank (1745-1821) kurzfristig von Sommer 1784 bis Ostern 1785 zu finden war, schloss sich an. Unter Johann Heinrich Fischer (1759-1814), der das Klinikum in Personalunion mit der Geburtshilfe leitete, erhielt die Einrichtung im Jahre 1786 die Bezeichnung „Königliches Clinicum zu Göttingen“.77

1792 übernahm dann Friedrich Benjamin Osiander die Leitung des königlichen Klinikums; doch es gelang auch ihm nicht, wieder an die alte Blütezeit anzuknüpfen. Im Durchschnitt besuchten nur noch sechs Studenten pro Semester das praktische Klinikum. ${ }^{78}$ Inzwischen gab es nämlich auch mehrere private Einrichtungen, die praktischen medizinischen Unterricht erteilten und dem königlichen Klinikum Patienten entzogen. Da sie mit einer Ausnahme alle ohne staatliche Zuschüsse arbeiteten, glaubte die Regierung, Geld einsparen zu können, wenn sie das königliche klinische Institut eingehen ließe. Der entsprechende Unterricht war ja durch die privaten Einrichtungen gewährleistet. Osiander musste sich mit diesem Regierungsbeschluss wohl oder übel einverstanden erklären. Im Sommersemester des Jahres 1802 war damit die erste Epoche der medizinischen Klinik an der Universität Göttingen beendet.

Die privaten klinischen Anstalten hielten sich jedoch nur wenige Semester. Als 1803 Karl Gustav Himly die Leitung des akademischen Hospitals übernahm, richtete er umgehend wieder ein ambulantes Stadtklinikum ein. Da im Hospital selbst zu wenig Krankheitsfälle für Demonstrationen vorhanden waren, war ohne ein 
solches Institut für ihn ein klinischer Unterricht nicht denkbar. Dieses Klinikum stand von nun an in enger Verbindung mit dem Hospital und hatte seinen rein internen Charakter verloren, da auch die chirurgischen Patienten mitbehandelt wurden. Die Regierung übernahm fortan die Kosten. Ab 1823 war die Einrichtung unter der Leitung von Wilhelm Heinrich Conradi wieder ein rein medizinisches Institut und eng mit der medizinischen Klinik verbunden. ${ }^{79}$ Daraus entwickelte sich dann die heutige medizinische Poliklinik. 


\section{Vom Gasthaus zum Großklinikum}

Wie die Ausbildung zum Arzt am gründlichsten und wirkungsvollsten durchgeführt werden sollte, dieser Gedanke spielte bei der Gründung der Medizinischen Fakultät eine ganz entscheidende Rolle. Im Vordergrund dieser Überlegungen stand dabei die Forderung nach einem praktisch-klinischen Unterricht am Krankenbett, wie es beispielsweise Leiden praktizierte. Da dafür entsprechende Hospitäler nötig waren, war deren Bau also unumgänglich.

\section{Das akademische Hospital}

Das ,akademische Hospital“ am Geismartor verdankte seine Existenz dem Zusammenwirken der Freimaurer-Loge „Augusta ₹u den drei Flammen " und der Universität. Als wesentliches Bindeglied galt der Freimaurer August Gottlieb Richter (17421812). Der Neffe von Georg Gottlob Richter, der in der Anfangsphase der Universität neben Haller Ordinarius an der Medizinischen Fakultät war, hatte unter der Obhut seines Onkels in Göttingen studiert und wurde 1764 promoviert. Nach einer längeren wissenschaftlichen Reise durch Westeuropa, auf der er mit den wichtigen zeitgenössischen Chirurgen zusammentraf, wurde er nach seiner Rückkehr 1766 zum Extraordinarius und 1771 zum Ordinarius ernannt. Mit seinen Göttinger Arbeitsbedingungen war Richter zunächst recht unzufrieden und klagte mehrfach darüber, dass ihm die Möglichkeiten zu Beobachtungen am Krankenbett fehlten und es ihm dadurch verwehrt sei, seine Ideen in der praktischen Erfahrung zu verifizieren. ${ }^{80}$ Und so war sein persönliches Interesse mitentscheidend für die entsprechenden Freimaurerinitiativen zum Bau eines Hospitals, die seit 1778 belegt sind. ${ }^{81}$ Nach mehreren Verhandlungsrunden mit der Regierung in Hannover konnte am 11. Januar 1781 unter der „Hammerfübrung“von Johann Benjamin Koppe (1750-1791) ein Haus für das geplante Hospital gekauft werden. ${ }^{82}$ Pütter bemerkt dazu rückblickend: „Noch fehlte bisher ein chirurgisches und KrankenHospital, um sowobl in der Wundarzney als der innerlichen Heilkunde mebr anschauende und selbstübende practische Anleitung geben zu können. Diesem Mangel abzubelfen ist seit dem Jahre 1780. vorerst ein am Ende der Stadt (unmittelbar am Geismarthore) gelegenes geräumiges Haus bestimmt. " 83 Bei dem von der Freimaurerloge gekauften Objekt handelte es sich um das ehemalige Gasthaus „Zu den sieben Thürmen“, das hinsichtlich seiner Lage und Bauweise allerdings nur bedingt den zeitgenössischen Vorstellungen 
eines funktionsgerechten Krankenhauses entsprach. Vor allem mangelte es an genügend frischer Luft und fließendem Wasser. „Man mußte froh sein, in Göttingen überbaupt ein Hospital zu baben, dessen Umfang und Struktur einigermaßen dem alten Leidener Muster entsprachen." ${ }^{84}$ Das zweistöckige Fachwerkhaus besaß zwei Säle, sieben Stuben, neun Kammern, eine Küche und war teilweise unterkellert. Der Garten hinter dem Haus hatte zwölf Fruchtbäume und erstreckte sich bis zum Wall. ${ }^{85}$

Da die durch acht eiserne Öfen besorgte Luftventilation nicht ausreichend genug war, mussten im Winter 1798/99 zwei weitere Windöfen zur Unterstützung eingesetzt werden. Auch war kein fließendes Wasser vorhanden; es wurde aus einem 27 Fuß tiefen Brunnen im Hof geschöpft. ${ }^{86}$ Probleme bereitete auch der Wasserabfluss; diese konnten erst durch die Änderungen der Straßenhöhe und Straßenneigung einigermaßen beseitigt werden. ${ }^{87}$

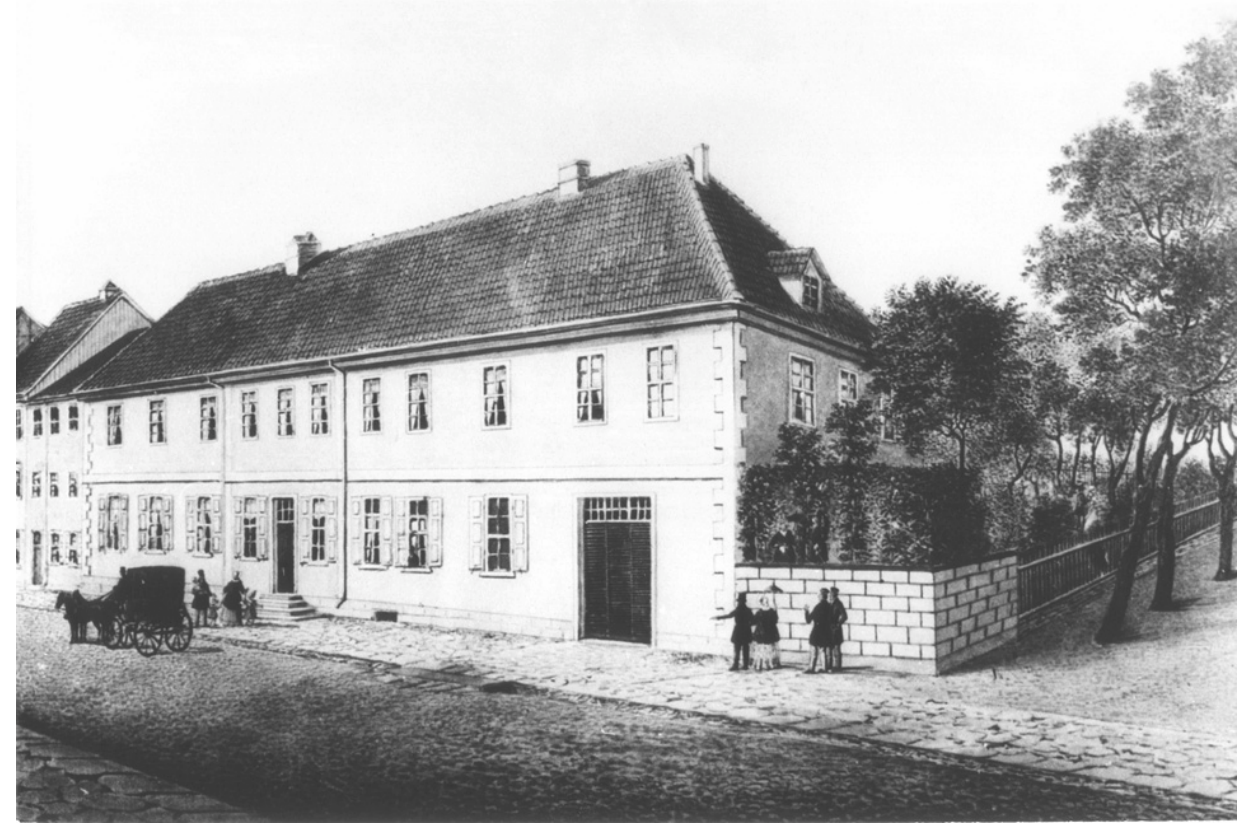

Abb. 8: Das ehemalige akademische Hospital um 1860 Anonymes Aquarell (heute: Kurze Geismarstraße)

Am 14. April 1781 konnte August Gottlieb Richter, der als bedeutendster Kopf unter den damaligen Medizinern und als „Klassiker"der Chirurgen galt und im Jahr zuvor zum Leibarzt ernannt worden war, in den "Göttingischen Gelehrten Anzeigen" die Eröffnung des Hospitals mit 15 Betten ankündigen: „Da die Einrichtung des 
hiesigen öffentlichen Hospitals gegen das Ende des April zu Stande gebracht seyn wird, so wird biemit bekannt gemacht, daß man vom ersten May an Kranke nicht allein aus der Stadt Göttingen selbst, sondern auch von den umliegenden Orten, in demselben aufnehmen, und sowohl mit allen Hülfsmitteln der Medicin und Chirurgie und Wäsche und Kleidung versehen, als auch mit Kost und Nahrung verpflegen wird. " 88

Nachdem Richter sein Ziel erreicht hatte, flaute sein Engagement deutlich ab. Wöchentlich nur zwei Stunden nachmittags soll er das Hospital besucht und von Zeit zu Zeit anfallende Operationen verrichtet haben. ${ }^{89}$ Durch ausgedehnte Konsultations- und Badereisen entzog er sich immer wieder über einen längeren Zeitraum seinen Göttinger Verpflichtungen, sodass sich die Fakultät daher mehrfach genötigt sah, ihn an seine Aufgaben zu erinnern und ihm sogar den Entzug der Bezüge anzudrohen. ${ }^{90}$

Richter selbst empfand seine Aufgabe als Hospitaldirektor zusehends als Last und bat um seine Dispension, der aber nicht entsprochen wurde. ${ }^{91}$ Erst die Vereinigung von Armenkrankenhaus ${ }^{92}$ und akademischem Hospital am 30. Mai 1797 führte zu einer strukturellen Neuordnung.

Johann Friedrich Strohmeyer (1750-1830), seit 1784 Hospitalarzt und Ordinarius, wurde in seiner Eigenschaft als Stadtphysikus und Mitglied der neu gebildeten Hospitalkommission zum zweiten Direktor des akademischen Hospitals befördert. Richter nutzte die neu geschaffene Situation umgehend zum ersehnten Rückzug und rechtfertigte sein Tun in einer sachlich begründeten Stellungnahme. ${ }^{93}$

Richter hat also seine ursprüngliche Forderung, die Theorie durch Praxis zu untermauern, durch die Aufgabe seiner praxisbezogenen Tätigkeit, nicht stringent aufrecht erhalten. Seiner Publikationstätigkeit war der Rückzug allerdings förderlich.

Das von ihm geschaffene wissenschaftliche Werk ist beachtlich. Neben seinen ophthalmologischen Studien, zu deren bedeutendste die ,Abhandlung von der Ausziehung des grauen Staares" (1773) zählt, stand die Chirurgie im Mittelpunkt seiner Forschung. Von 1771-1797 erschienen insgesamt 15 Bände der „Chirurgischen Bibliothek", einem kritisch referierenden Journal, dessen Beiträge Richter weitgehend selbst verfasste. Geradezu klassisch und für die Chirurgie richtungsweisend wurde seine „Abhandlung von den Brüchen“ (1779) und die „Anfangsgründe der Wundarzneykunst" (1782-1809), deren einzelne Bände bis zu vier Auflagen erreichten und in mehrere Sprachen übersetzt wurden. Posthum erschienen „Medicinische und chirurgische Bemerkungen“(1793-1813) und die „Specielle Therapie“(1813-1836). Zu Richters bleibendem Verdienst zählt die Mehrung des chirurgischen Ansehens im 18. Jahrhundert. Durch die Annäherung der Chirurgie an die Medizin durch den von ihm propagierten klinischen Unterricht stellte er das handwerklich ausgerichtete Fach auf eine wissenschaftliche Grundlage und in vielleicht noch höherem Maße gilt dies für die operative Seite der Augenheilkunde. ${ }^{94}$ 


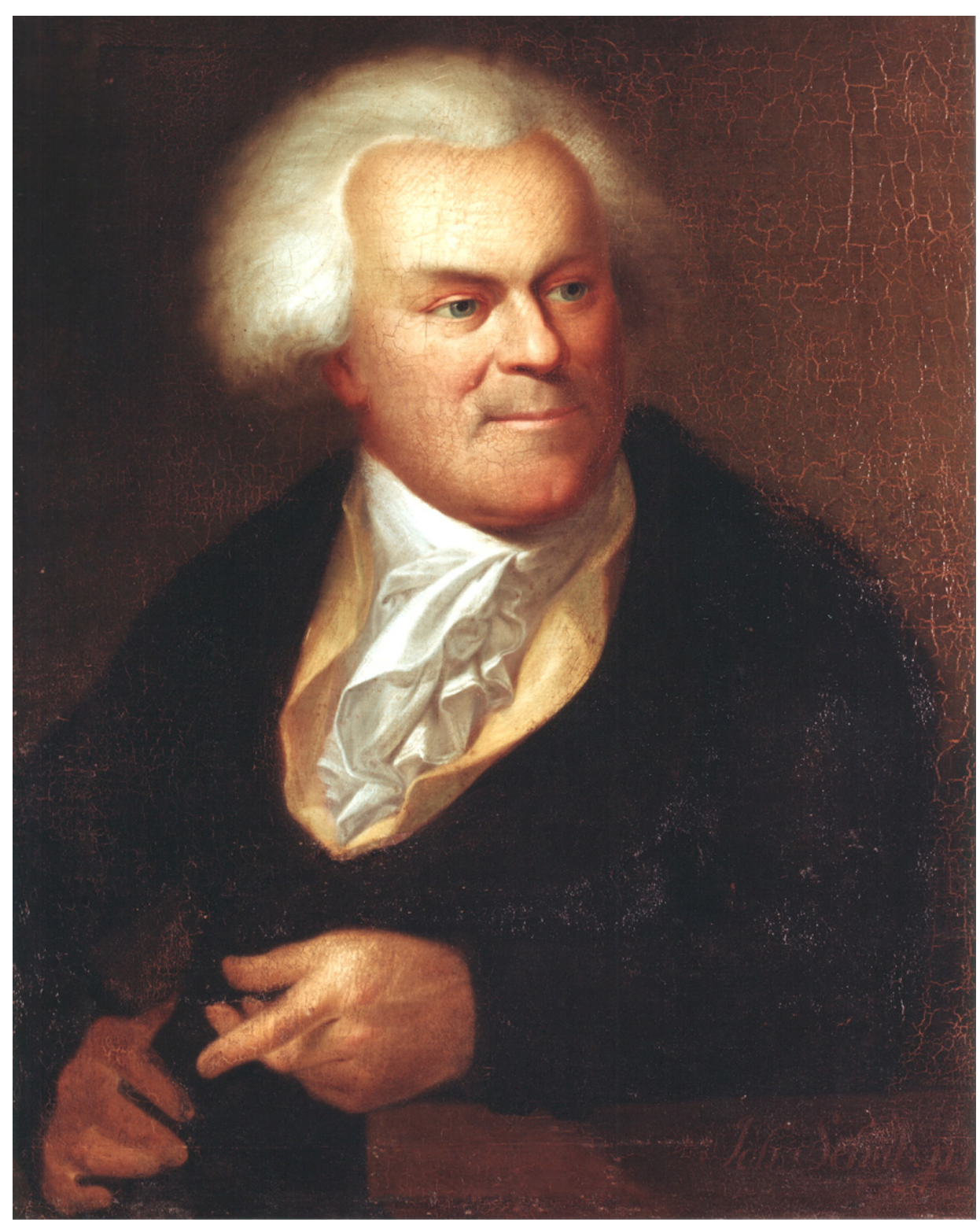

Abb. 9: August Gottlieb Richter

Öl auf Leinwand von Johann Schulz, o. J. 
Über die Aufnahme der Patienten hatte Richter bereits in der Eröffnungsankündigung seine Vorstellungen festgelegt. Aufgenommen sollten alle werden, bei denen ein chirurgischer Eingriff oder ,innere Arzneyen “ nötig waren. Diejenigen, die nicht einer raschen Hilfe bedurften, mussten sich zunächst vorstellen. Richter nannte in anderem Zusammenhang die Absicht, die hinter dieser Bestimmung stand. „Ich habe die Freyheit und Gelegenheit, die Kranken zu wählen; und unter denen, die sich presentiren, wähle ich die wichtigsten, und lehrreichsten. "95 1793 veröffentlichte Richter eine Sammlung von Krankengeschichten, die wohl repräsentativ für die im Hospital erfolgten Behandlungen ist. Da er darin lediglich Symptome aufzählte, zeigte Richter, dass sein Interesse in erster Linie dem therapeutischen Erfolg galt und weniger der Diagnose. „Die Natur hat viele Wege, die der Anatom nicht kennt. Und wozu würde es bey der Behandlung der Krankheit nutzen, sie zu kennen. " 96

Nach Richters Rückzug aus der täglichen Klinikarbeit, ohne dass er allerdings von seinem Direktorenamt bis zu seinem Tode offiziell zurücktrat, trug der zweite Direktor Strohmeyer die Hauptlast. Doch auch er verließ nach drei Jahren das Hospital und widmete sich nur noch seiner Praxis. In der Folgezeit kam es, bedingt durch die oft sehr lange Abwesenheit Richters, zu Interimslösungen. 1802 1803 leitete der Geburtshelfer Friedrich Benjamin Osiander (1759-1822) in Personalunion neben der Gebärklinik auch das akademische Hospital. Für den chirurgischen Bereich holte er Konrad Johann Martin Langenbeck.

Im April 1803 wurde Karl Gustav Himly (1722-1837) neben Richter neuer Hospitaldirektor. Mit ihm begann die letzte Phase im alten Hospitalgebäude. Himly studierte zunächst am anatomisch-chirurgischen Kollegium seiner Heimatstadt Braunschweig, dann in Göttingen, wo er 1794 promoviert wurde. Nach seiner Tätigkeit in Feldspitälern trat er 1795 ins Braunschweiger „Collegium Carolinum medicum" ein, wo er als Professor und Direktor des Armenkrankenhauses Pathologie und Chirurgie lehrte. 1801 folgte er dem Ruf nach Jena und vertrat dort neben der theoretischen Medizin auch die Chirurgie und Augenheilkunde. Sein wissenschaftliches Werk umfasst neben zahlreichen ophthalmologischen Arbeiten auch die vom Brownianismus beeinflusste Schrift „Über die Wirkung der Krankheitsreize auf den menschlichen Körper" (1795), das mit naturphilosophischen Spekulationen durchsetzte „Lehrbuch der praktischen Heilkunde enthaltend allgemeine Nosologie, Heilmittellehre und Therapie“ (1807) sowie Studien „Über den Brand der weichen und harten Theile nebst einigen Grundzügen der medicinischen Theorie“ (1800) und „Über das Impfen der Kuhblattern" (1801). ${ }^{97}$

Himlys Amtsführung war von Anfang an durch die immer schärfer werdenden Auseinandersetzungen mit dem Hospitalwundarzt Langenbeck überschattet. Schon während der Interimslösung unter Osiander hatte dieser versucht, seinen Anspruch durchzusetzen, die chirurgischen Patienten ausschließlich in eigenständiger Verantwortung zu behandeln. Auch unter Himly hielt er hartnäckig an dieser Forderung fest, was zu immer neuen Spannungen führte. Die Bemühungen Hannovers, 
durch Festlegung und Abgrenzung der jeweiligen Befugnisse verbunden mit entsprechenden Gehaltszulagen schlichtend einzugreifen, blieben jedoch ohne Erfolg. ${ }^{98}$

Im Frühjahr 1807 kam es schließlich zum endgültigen Bruch. Die dabei abgegebenen Stellungnahmen der beiden Kontrahenten gegenüber der Regierung spiegelten im Grunde neben menschlichen Schwächen und organisatorischen Querelen die zeitgenössische Auseinandersetzung um chirurgische und medizinische Fachinhalte wider. ${ }^{99}$ Langenbeck schied aus dem Hospital aus und errichtete noch im selben Jahr am Wilhelmsplatz sein eigenes „Chirurgisches Hospital“, für das er sich wohl vorbereitet bereits einen beachtlichen staatlichen Zuschuss gesichert hatte. ${ }^{100}$

Himly hatte von Anfang an über die schlechte räumliche Situation des akademischen Hospitals geklagt. Die Krankenzimmer seien niedrig, eng und dunkel, die Luft schlecht; ferner gebe es keine Möglichkeiten, „gefährliche" Kranke zu isolieren, um Ansteckungen zu verhindern. Auch die allgemeinen hygienischen Verhältnisse seien insgesamt katastrophal; Wanzen und anderes Ungeziefer seien nicht auszurotten und die zur Verbesserung der Räumlichkeiten begonnenen Bauvorhaben blieben unvollendet. ${ }^{101}$

\section{Himlys Hospital am Leinekanal}

Es erschien daher nicht verwunderlich, dass Himly auf eine neue Lösung drängte und nach einem neuen Hospitalgebäude Ausschau hielt. Im November 1809 kaufte er schließlich ein Haus am Stumpfebiel, das unmittelbar an sein Wohnhaus angrenzte. Im Vergleich zum alten Hospital war dieses neue Gebäude, das aus einem Mittelteil und zwei längeren Seitenflügeln bestand, nicht nur in räumlicher Hinsicht ein erheblicher Fortschritt. Im Erdgeschoss dieses dreistöckigen Gebäudes waren ambulatorische Klinik und Badeanstalt; im Obergeschoss ein Ausstellungsraum mit einer Knochensammlung und Wohnräume für Bedienstete, darunter auch vier Wohnungen für Ärzte.

Kernstück war jedoch die mittlere Etage. Dort befanden sich je ein großer Saal für Männer und Frauen, zwei Zimmer für Augenkranke, die einfarbig dunkelgrün gestrichen waren und durch Vorhänge stark abgedunkelt werden konnten, zwei Isolierzimmer für Venerische, Krätzige und Krebskranke, eines für Patienten, die von ansteckenden, hitzigen Krankheiten befallen waren, und zwei Zimmer für frisch Operierte. In der Mitte lag das sehr helle Operationszimmer. Etwas abgesondert gab es noch zwei geräumige Zimmer für Kranke höheren Standes; sie waren auch für reiche auswärtige Kranke offen.

Durch seine Lage boten sich dem neuen Hospital wesentlich günstigere Möglichkeiten. Vor allem war nun fließendes Wasser vorhanden, das aus dem 150 Fuß entfernten Leinekanal durch eine verdeckte Rohrleitung zu einem nahe am Haus liegenden Wasserbehälter gebracht wurde. Ein großer Garten schirmte die Kranken gegen die Öffentlichkeit und Umgebung ab. ${ }^{102}$ 


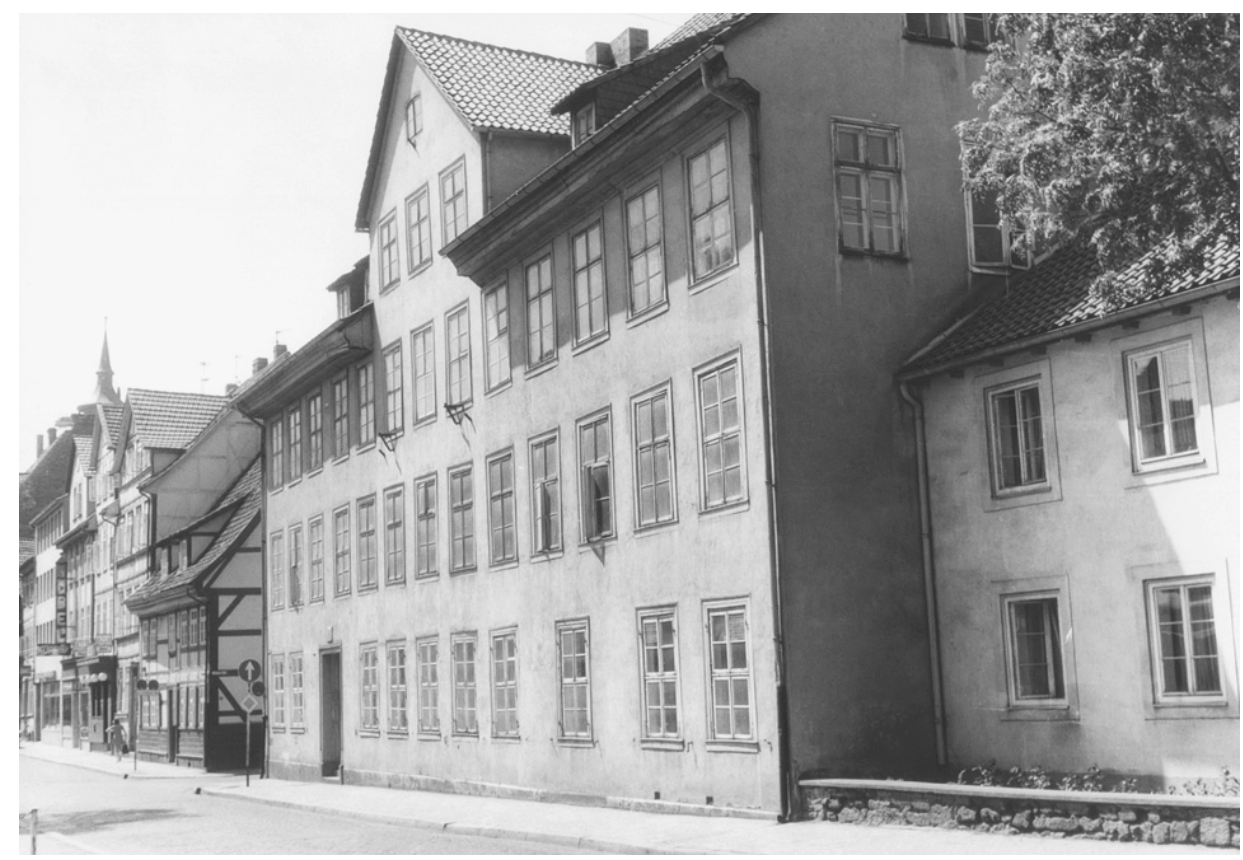

Abb. 10: Himlys Hospital am Stumpfebiel Photographie aus dem 20. Jahrhundert

Von Anfang an lag es nicht in Himlys Absicht, den Patientenkreis auf ausschließlich medizinische Fälle, trotz des in Göttingen vorhandenen eigenen chirurgischen Hospitals, zu beschränken. Eine solche Trennung hielt er für widersinnig, da sie einer vernünftigen klinischen Ausbildung entgegenstand. Insgesamt verfügte das Hospital über 25 Betten. Behandelt wurde das gesamte medizinische Spektrum. Von den im Jahre 1810 stationär behandelten Krankheiten betrafen die häufigsten Fälle die Augenheilkunde; hier standen Staroperationen und künstliche Pupillenbildungen deutlich im Vordergrund. Die übrigen Fälle reichten von Amputationen, Wechselfieber, Pneumonien, Typhus, akuten Gichtanfällen und Fußgeschwüren bis hin zu venerischen Krankheiten. Einmal entband er eine „venerische Person“, ohne dass das Kind infiziert wurde. Gliederlähmungen, Taubheit und auch Augenkrankheiten wurden mit Elektrizität, der galvanischen Batterie, behandelt. ${ }^{103}$

28 Jahre leitete Himly ,sein“ Hospital. Die letzten Jahre waren allerdings überschattet durch gravierende Vorwürfe, die ihm als Person sowie seiner Funktion als Leiter des Hospitals galten. So habe seine Trunksucht mehrfach Ärger erregt, für Arzneien würde das Geld planlos verschwendet und die Pflege der Kranken sowie die hygienischen Verhältnisse des Hospitals seien katastrophal. ${ }^{104}$ Völlig aus der Luft gegriffen waren die Vorwürfe nachgewiesenermaßen allerdings nicht. ${ }^{105}$ Himly 
selbst hatte mehrfach auf seine angegriffene Gesundheit hingewiesen und vor allem über Gelenkschmerzen geklagt. Auch scheint er unter Depressionen gelitten zu haben, wie aus einer Notiz Langenbecks hervorgeht. „Am Tage vor seinem Ende ließ er mich rufen und verlangte über Facultätsangelegenheiten Rath und Beistand von mir. Ich konnte ibm beides ertheilen, fand ihn aber so sehr gemütskrank, daß ich nicht obne Sorgen wegging. " 106 Am 22. März 1837 ertrank Himly in der Leine hinter dem St. Marien Kirchhof.

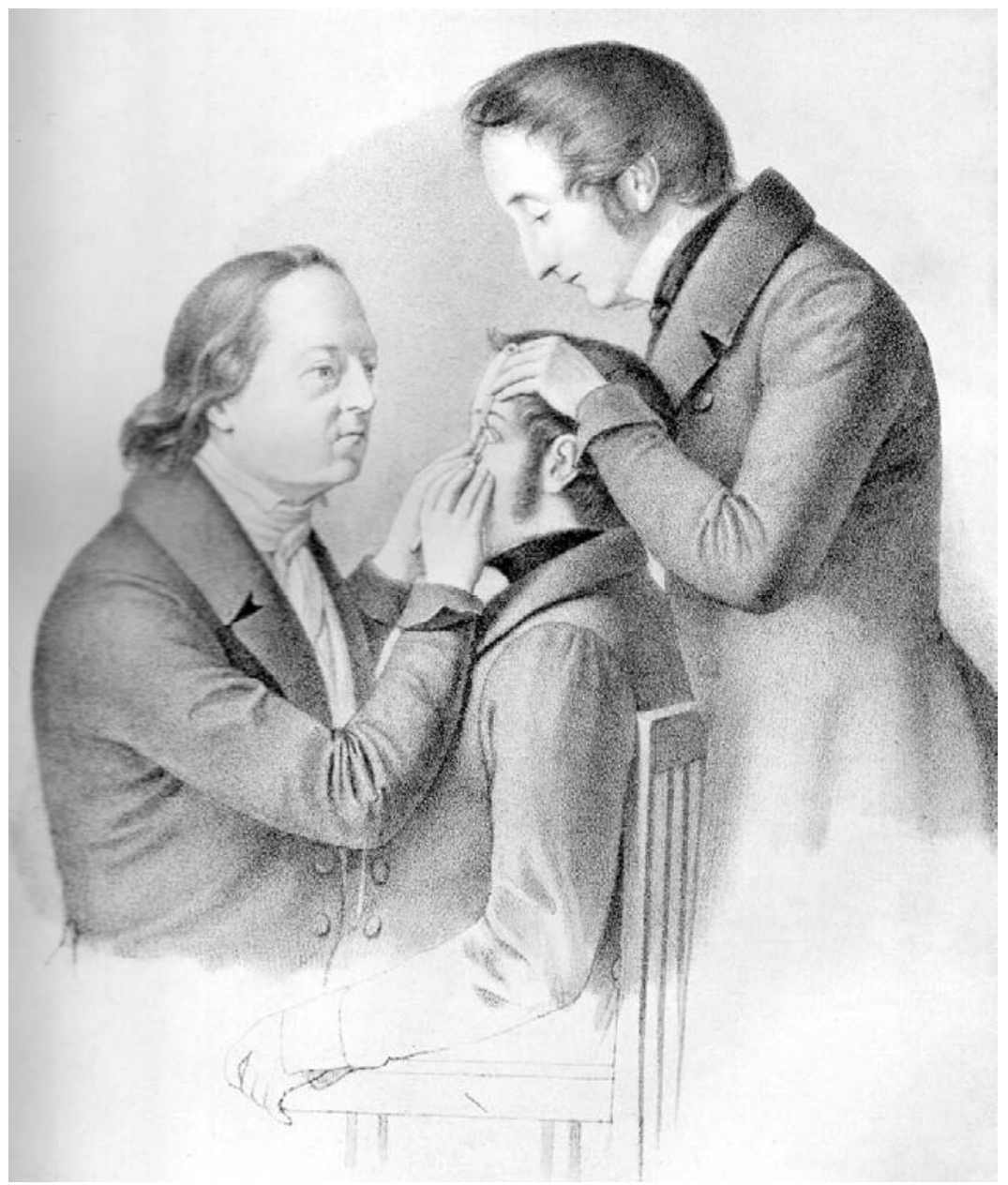

Abb. 11: Karl Gustav Himly und Christian Georg Theodor Ruete bei einer Augenoperation (Starstich)

Aus: Julius Hirschberg, Geschichte der Augenheilkunde. Leipzig 191 1, Tafel I 
Nach Himlys Tod wurde der Hofrat und Professor Johann Wilhelm Heinrich Conradi (1780-1861) der letzte Direktor des Hospitals am Stumpfebiel. Er war bereits 1823 als weiterer Lehrer für allgemeine Therapie berufen worden. Conradi stammte aus Marburg, hatte dort Medizin studiert, wurde 1802 promoviert und war maßgeblich beim Aufbau des akademischen Krankenhauses im ehemaligen Elisabeth-Hospital beteiligt. 1813 übernahm er dessen Leitung, ging aber bereits ein Jahr später nach Heidelberg, um dort die Medizinische Klinik einzurichten. ${ }^{107}$ 13 Jahre lang hat Conradi das akademische Hospital geleitet. Wesentlich neue Entwicklungen hat er allerdings nicht bewirkt und nahm auch keinen Anteil an den zu seiner Zeit begonnenen Planungen des ersten Neubaus für ein Universitätskrankenhaus, in dem das akademische und chirurgische Hospital Langenbecks, die seit 1838 aus acht Betten bestehende stationäre Abteilung im Rahmen der Poliklinik, die von Conrad Heinrich Fuchs (1803-1855) geleitet wurde, und die Klinik für Sinneskranke (Augen- und Ohrenkranke), der Christian Georg Theodor Ruete (1810-1867) vorstand, unter einem Dach zusammengefasst werden sollten. Aber seine beiden Lehrbücher „Allgemeine Pathologie"1811 und „Specielle Pathologie“ 1811 fanden entsprechende Resonanz und erreichten mehrere Auflagen.

\section{Ernst-August-Hospital}

Bei einem Besuch in Göttingen am 1. Juli 1845 überzeugte sich der hannoversche König Ernst August (1771-1851) selbst von den unhaltbaren Zuständen in Himlys Hospital und sicherte den Bau eines neuen zu. ${ }^{108}$ Als Bauplatz wurde das Gebiet des ehemaligen Tuchrähmenhofs und des Geistgartens ausgewählt und 1846 der Stadt Göttingen abgekauft. ${ }^{109}$ Die Wahl dieses Standorts, bei der der Wunsch des Königs und das Bemühen um größtmögliche Sparsamkeit mehr Gewicht besaßen als medizinische Überlegungen, stellte sich jedoch sehr bald als falsch heraus. Die Feuchtigkeit des Terrains, die trotz umfangreicher zusätzlicher Baumaßnahmen nicht behoben werden konnte, brachte von Anfang an erhebliche Probleme mit sich und bereits nach kurzer Zeit fehlte der Raum für notwendige Erweiterungen.

Am 9. Oktober 1850 nahm das neue akademische Krankenhaus seinen Betrieb auf. Die offizielle Übergabe, die sich König Ernst August vorbehalten hatte, erfolgte dann in seiner Anwesenheit am 6. August 1851. Seit diesem Zeitpunkt führte das Krankenhaus offiziell den Namen „Ernst-August-Hospital“. ${ }^{110}$ Das Hauptgebäude mit seiner schlichten klassizistischen Fassade hatte einen zurückspringenden Mittelteil, zwei Flügel und zwei Stockwerke mit einem hohen Unterbau zum Schutz gegen die Feuchtigkeit. In Hintergebäuden auf dem Hof waren das Pathologische Institut, der Sektionssaal und die Totenkammer untergebracht. Das Isolierhaus befand sich im Garten auf der anderen Straßenseite. Die gesamte Konzeption der Anlage war detailliert durchdacht und brachte krankenhausbauliche Aspekte mit den Bedürfnissen des Krankenhausbetriebes überzeugend in Ein- 
klang. Das untere Stockwerk teilten sich die medizinische Abteilung und die Poliklinik, das obere die Chirurgie und die Abteilung für Sinneskranke (Augenklinik). In den beiden Flügeln wurden Männer und Frauen getrennt untergebracht. Die Versorgungsanlagen befanden sich im Unterbau. In jedem Stockwerk gab es vier große Krankensäle mit jeweils zwölf und vier kleinere mit sechs Betten. In der medizinischen Klinik im Erdgeschoss waren für Privatpatienten fünf, in der chirurgischen Klinik im ersten Stock drei und für kranke Studenten ein Zimmer vorgesehen. Für den Unterricht am Patienten stand in der Poliklinik ein funktionsgerechter halbrunder Saal zur Verfügung; der entsprechende Raum bei der Chirurgie in der Etage darüber wurde als Operationssaal genutzt. ${ }^{111}$ Ein „Hospitalvorstand", der aus den Direktoren der drei Kliniken bestand, war für den klinischen Bereich zuständig. Die technische Organisation lag in der Hand eines Verwalters.

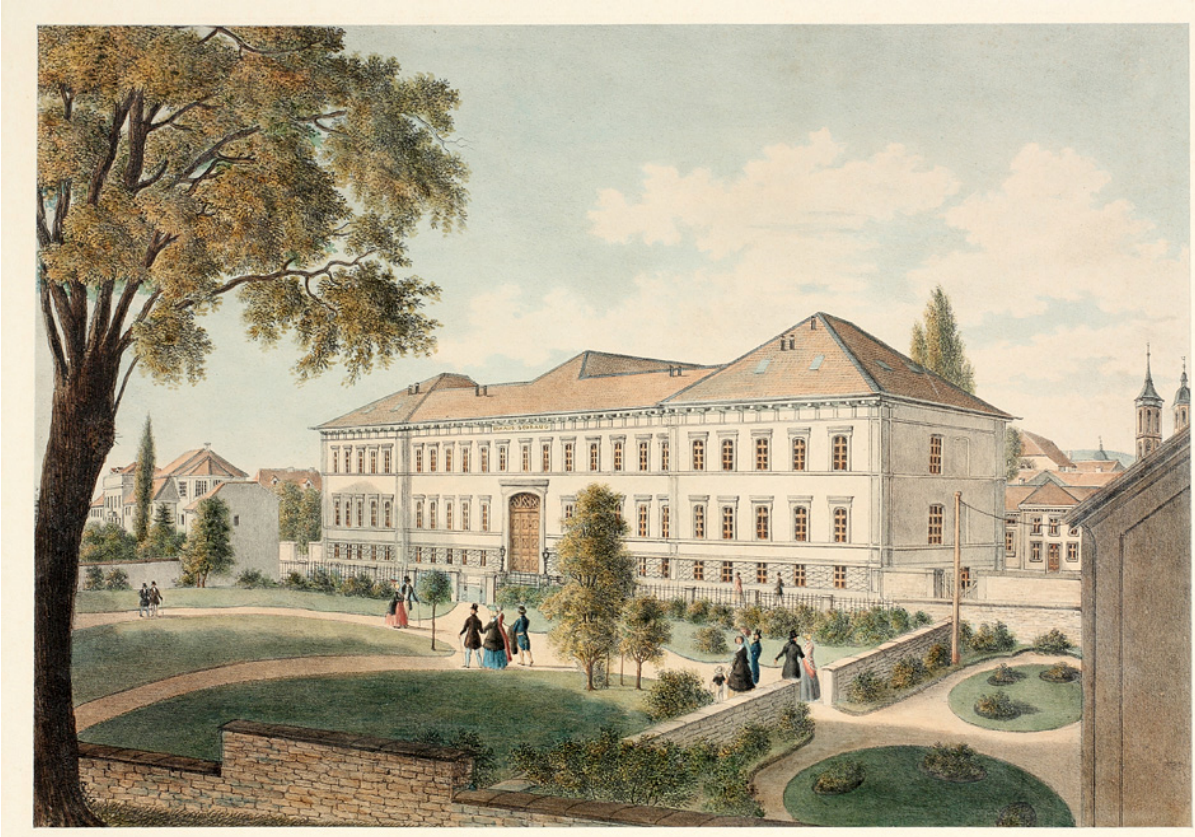

Abb. 12: Das Ernst-August-Hospital in der Geiststraße Farbige Lithographie von Friedrich Besemann, um 1852

Die Patienten stammten aus Göttingen und der ländlichen Umgebung. Aufgrund der insgesamt schlechten hygienischen Verhältnisse, vor allem die Wasserversorgung und -entsorgung waren in Göttingen katastrophal, war ein Großteil der Patienten Opfer von Epidemien. Besondere Vereinbarungen gab es über die Behandlung von kranken Eisenbahnarbeitern und erkrankten Soldaten. ${ }^{112}$ Im Vergleich 
mit dem zeitgenössischen Niveau des Krankenhausbaus hatte das Ernst-AugustHospital einige recht beachtliche Fortschritte aufzuweisen. An erster Stelle sei hier das Isolierhaus für ansteckende Kranke zu nennen. Das sogenannte „Blatternhaus“ war eine der ersten derartigen Einrichtungen in Deutschland. Ferner zählten dazu die Wasserklosetts, das Ableiten der Abwässer durch ein Röhrensystem und die Luftreinigung mit Hilfe von Öfen, zu öffnenden Teilfenstern und Luftschächten, die aber nicht immer zur Zufriedenheit funktionierten. ${ }^{113}$

Zu den bereits nach der Erbauung bekannten Nachteilen kam im weiteren Verlauf ein sich ständig vergrößernder Mangel an spezifischen Räumen. Dies führte zu Zwischenlösungen, die teilweise recht provisorischen Charakter besaßen. 1861 wurde das Hintergebäude, in dem sich die Pathologie befand, aufgestockt. ${ }^{114}$ Als im Winter 1865/66 gehäuft Typhusfälle auftraten, wurde im Garten ein Krankenzelt aufgestellt und 1867 durch ein zweites ergänzt. ${ }^{115}$ Beide blieben bis Sommer 1874 in Gebrauch und wurden im folgenden Jahr durch eine Baracke ersetzt. ${ }^{116}$

Ein weiteres Problem schuf die Patientenzunahme vor allem in der Augenklinik. Durch die Teilauslagerung in das in unmittelbarer Nähe liegende Wrisbergsche Haus wurde 1869 zunächst eine Lösung gefunden. Doch reichte auch diese Maßnahme nach einiger Zeit nicht mehr aus und 1873 wurde deshalb neben dem Ernst-August-Hospital ein Neubau für die Augenklinik errichtet und Anfang August 1873 eröffnet. ${ }^{117}$ Sie hatte vier Säle mit jeweils sechs Betten, zwei mit drei Betten und vier Einzelzimmer für Privatpersonen. ${ }^{118}$ Wiederum war die Raumkapazität nach einigen Jahren erschöpft, sodass 1879 erneut im Wrisbergischen Haus und 1890 im nun verlassenen Ernst-August-Hospital Dependancen eingerichtet wurden. Bis 1906 blieb die Augenklinik in diesen Räumen. ${ }^{119}$

Von Anfang an hatte das Ernst-August-Hospital mit Unzulänglichkeiten zu kämpfen, die weiter zunahmen. Die Forderungen nach einem Neubau wurden deshalb immer dringender. Hinzu kam, dass die enormen Entwicklungen, die sich in der Medizin des 19. Jahrhunderts sowohl in ihren naturwissenschaftlichen Grundlagen als auch in ihrer klinischen Spezialisierung vollzogen, an ein Krankenhaus neue Anforderungen stellten, denen das Ernst-August-Hospital nicht mehr gewachsen war. Bereits 1877 stellte die Medizinische Fakultät offiziell den Antrag für einen Neubau des Hospitals und des Pathologischen Instituts. 1879 wurde im Weender Feld am sogenannten Kirchweg ein geeignetes Grundstück gefunden. ${ }^{120}$

Die wichtigsten Lehrer und Direktoren am Ernst-August-Hospital waren die Chirurgen Wilhelm Baum (1799-1883) und Franz König (1832-1910), der Ophthalmologe Theodor Leber (1840-1917) sowie in der Medizinischen Klinik Carl Ewald Hasse (1810-1902) und vor allem Wilhelm Ebstein (1836-1912), der durch sein wissenschaftliches Werk besonders herausragte und als entschiedener Verfechter eines Klinikneubaus galt. Ebstein hatte in Breslau und Berlin studiert und wurde 1859 promoviert. 1877 kam er nach Göttingen und leitete zunächst eine 
Poliklinik, ehe er 1877 die Direktion der medizinischen Klinik und Poliklinik des Ernst-August-Hospitals übernahm. Sein wissenschaftliches Werk deckt fast vollständig das gesamte Gebiet der inneren Medizin ab. Gewisse Schwerpunkte bilden die Arbeiten zum Stoffwechsel, zu Leber und Niere, zu den Infektionskrankheiten sowie zu Herz und Gefäßen. Bis heute wird die Missbildung der Trikuspidalklappe in der rechten Herzkammer als „Ebstein-Anomalie“"bezeichnet. Auch zur Geschichte der Medizin hat Ebstein Grundlegendes vorgelegt, beispielsweise seine Studien zur Medizin im Alten und Neuen Testament sowie im Talmud. ${ }^{121}$

\section{Die Kliniken an der Humboldtallee}

Eine wesentliche Forderung, die die medizinische Wissenschaft am Ende des 19. Jahrhunderts an einen universitären Krankenhausbetrieb stellte, war die Zusammenführung der unterschiedlichen Teilkliniken an einem zentralen Ort. Der Ankauf des großflächigen Grundstücks von circa acht Hektar am Kirchweg (heute Humboldtallee) bot für die entsprechende Realisierung alle Voraussetzungen. Und es dauerte knapp zwei Dekaden, um die Forderung zu erfüllen. 1889 eröffneten

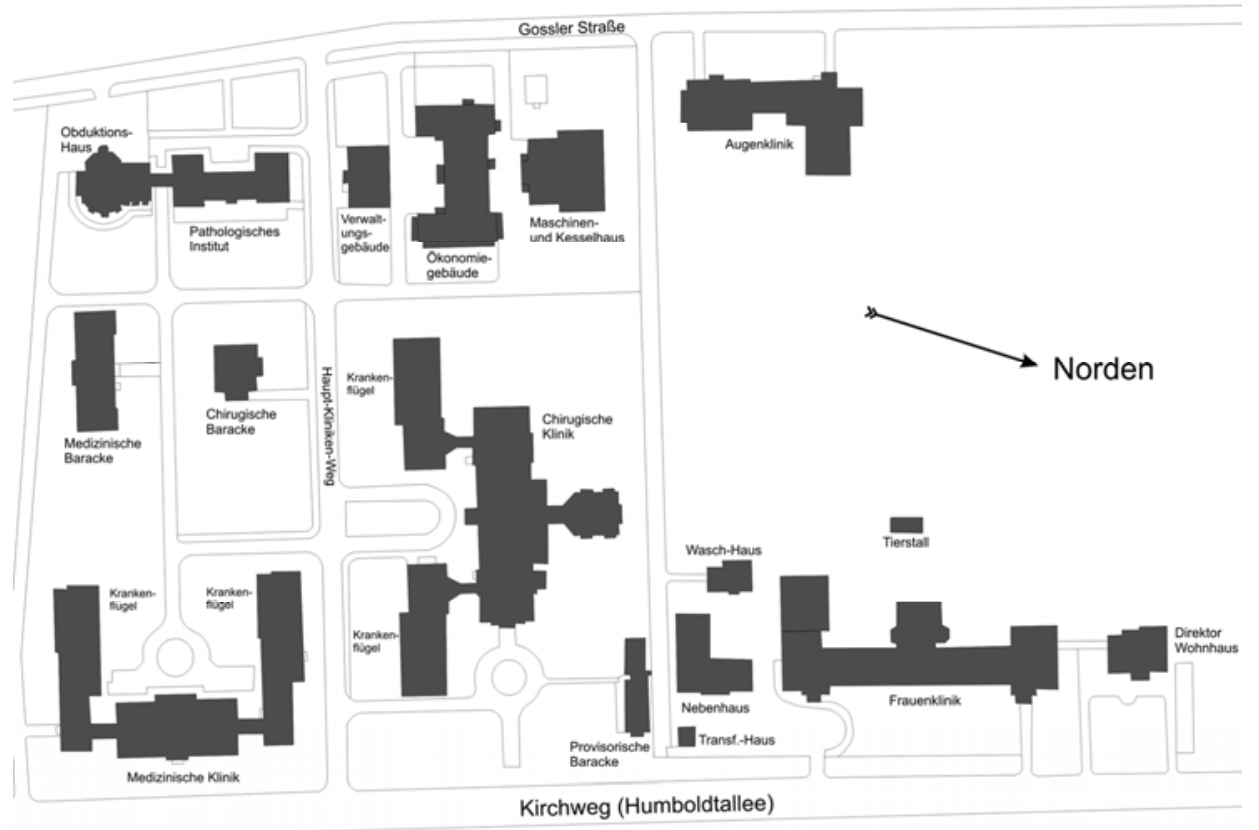

Abb. 13: Die Kliniken an der Humboldtallee 1908 
die Chirurgische, 1891 die Medizinische und 1896 die Frauenklinik ihren jeweiligen Neubau. 1906 folgte die Augenklinik. Das ebenfalls neu erbaute Pathologische Institut, das funktionsgerecht gleichfalls auf dem Terrain angesiedelt worden war, begann seine Arbeit bereits 1891. Hinsichtlich des architektonischen Entwurfs, der technischen Ausführung und der verwendeten Baumaterialien geben die Gebäude des gesamten Klinikbereichs ein einheitliches und recht gelungenes Bild ab. Die Konzeption der vier Teilkliniken ging von der Überlegung aus, den praktischen Krankenhausdienst mit dem medizinischen Unterricht und der wissenschaftlichen Forschung in einen vernünftigen Einklang zu bringen.

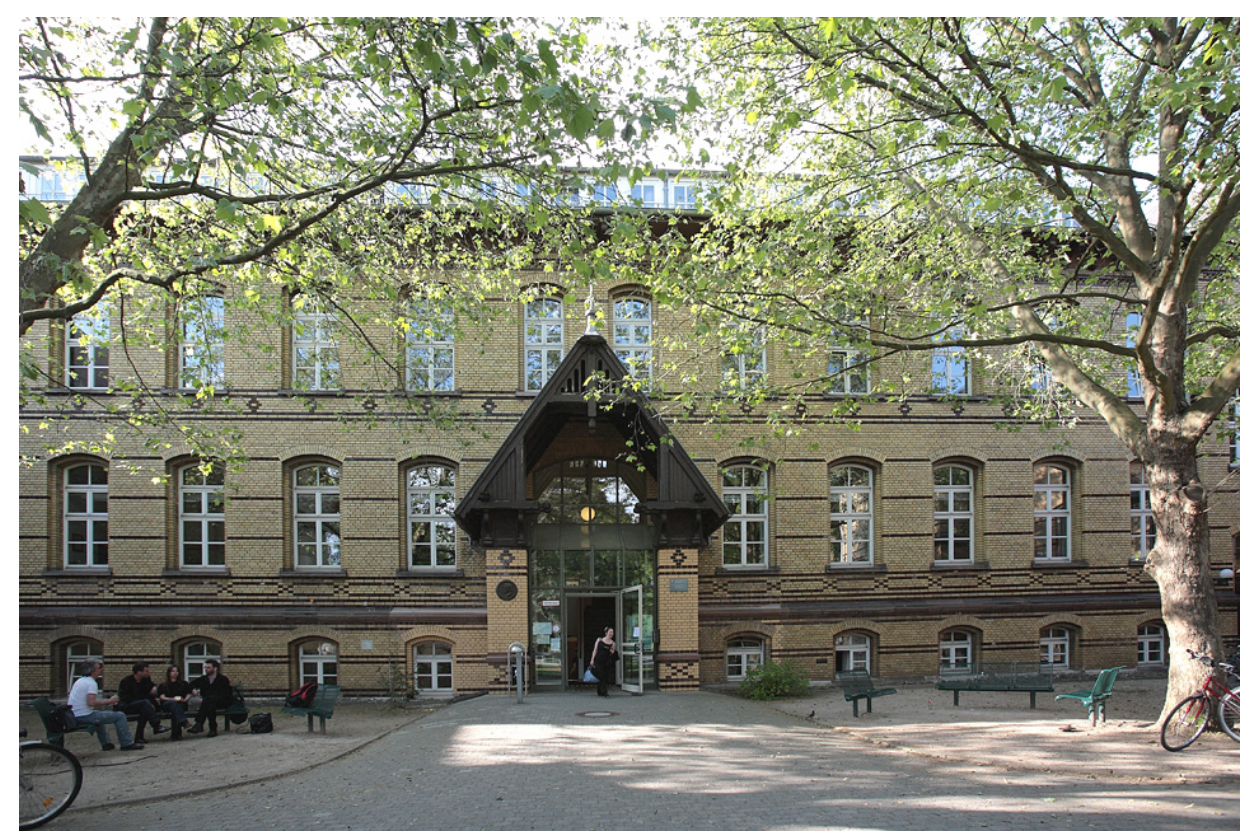

Abb. 14: Die Chirurgie (1889-1978)

(Südansicht des Mittelbaus)

Aufnahme 2009 (heute: Käte-Hamburger-Weg 3)

\section{Chirurgische Klinik}

Das zweistöckige Gebäude bestand aus einem zentralen Mittelbau mit einem an der Mitte angehängten elliptisch geformten Operationssaal und zwei flügelartigen Pavillons. Insgesamt hatte die chirurgische Klinik 109 Betten, die sich auf zwei Krankensäle und ein Zimmer für Frischoperierte im Erdgeschoss und auf vier Krankensäle, ein Isolier- und ein Delirantenzimmer im ersten Stock verteilten. Auf der Südseite erstreckte sich längs des Krankensaals und des sich daran anschlie- 
Benden Tagesraums eine überdachte Veranda. Für den Transport der Kranken diente ein mit Wasserdruck angetriebener Fahrstuhl. Die Ventilation in den Krankenräumen erfolgte durch eine zentrale Wasserdampfheizung, durch Luftklappen, die frische Luft ansaugten, und über einen Abluftkanal, der verbrauchte wieder abgab, durch besondere Vorrichtungen an den Fenstern und in den oberen Sälen durch Dachfirstventilatoren.

Zentrales Kernstück war die Anlage des Operations- und Lehrraums. Da beide Räume eigene Zugänge hatten, konnten sie mit Leichtigkeit aseptisch gehalten werden. Der halb elliptisch geformte Operationssaal war mit hohem Seiten- und Oberlicht ausgestattet, um Schatten über dem Operationsgebiet zu verhindern, und bot ausreichend Platz, um bequem an zwei Tischen nebeneinander zu operieren. Der Zuschauerraum mit seinen vier steilen Emporen für etwa 120 Studierende war so angelegt, dass alle eine bestmögliche Sicht auf die Operationen und die dabei erfolgten Demonstrationen hatten. Die Poliklinik, die neben dem Operationssaal lag, war räumlich so ausgestattet, dass etwa 5000 Patienten pro Jahr behandelt und gleichzeitig 80 bis 100 Studierende dabei unterrichtet werden konnten.

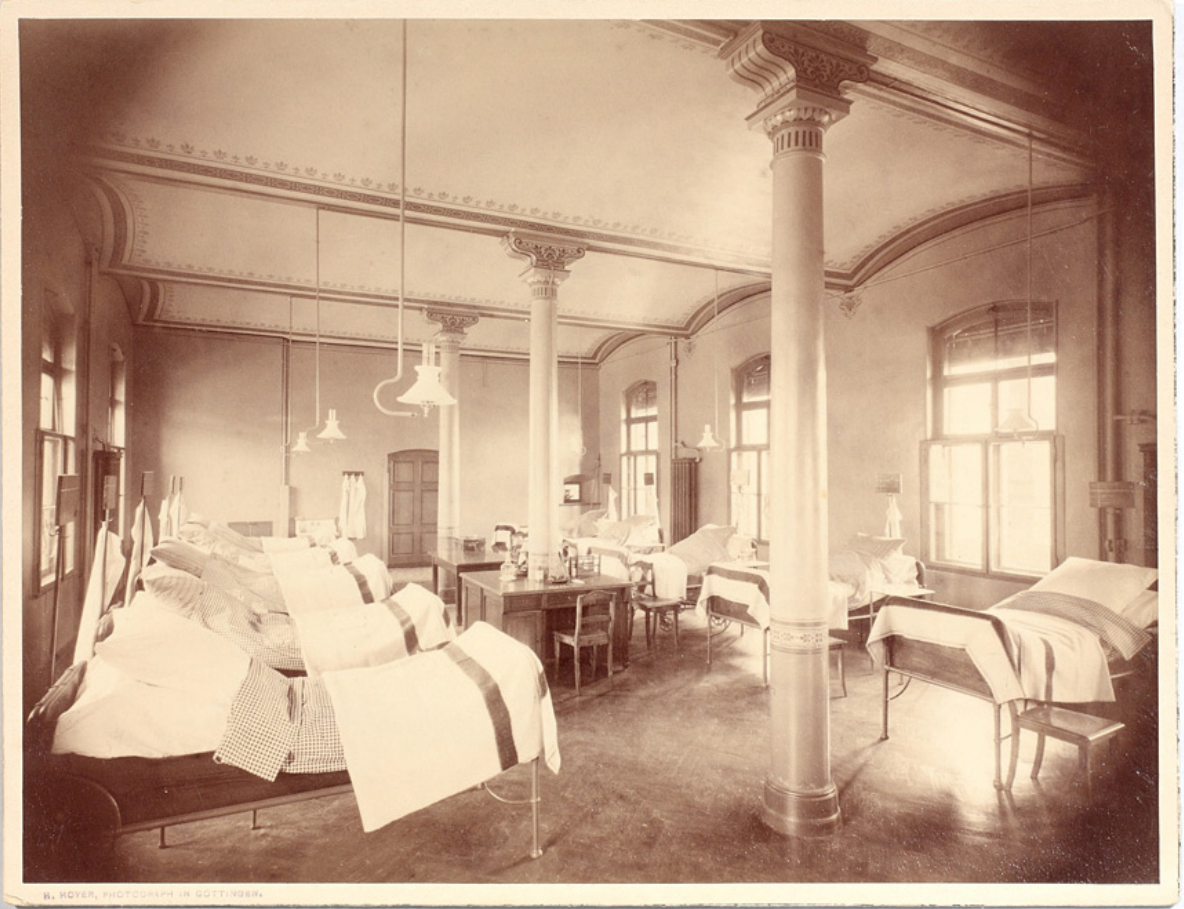

Abb. 15: Krankensaal in der Chirurgie aus dem Jahre 1889 
Die reichlich vorhandenen Funktionsräume, unter denen sich ein chemisch-bakteriologisches Laboratorium, das neben seinen spezifischen Aufgaben auch zu anatomischen Untersuchungen und zu Tierversuchen diente, und ein Auditorium für 80 bis 100 Zuhörer befanden, waren so angelegt, dass jederzeit ein optimales Arbeiten möglich war.

Die Kellerräume, die allerdings nur im Mittelbau vorhanden waren, dienten in erster Linie zur Aufbewahrung medizinischen Materials. Neben einer kleineren Portierswohnung gab es dort auch ein aus einem Speisesaal, Lese- und Billardzimmer und Bad bestehendes Lokal für die Ärzte der klinischen Institute. ${ }^{122}$

\section{Medizinische Klinik}

Auch die Konzeption der medizinischen Klinik folgte dem Prinzip einer Trennung des Krankenbereichs vom Lehr- und Funktionsbereich. Die dreigeschossige Anlage bestand aus einem Mittelbau, an den sich rechts und links zwei Seitenflügel anschlossen. Hier waren in jedem Stockwerk jeweils ein Tagesraum und ein großer Krankensaal, an den sich eine offene Halle zur Behandlung akuter wie chronischer Krankheiten anschloss, untergebracht. Im Mittelbau selbst befanden sich lediglich vier kleinere Zimmer für Privatpatienten bzw. für kranke Studierende und ein Zimmer mit vier Betten für klinisch kranke Männer. Insgesamt war die medizinische Klinik für 96 Betten berechnet. Die Poliklinik war gleichfalls im Mittelbau untergebracht.

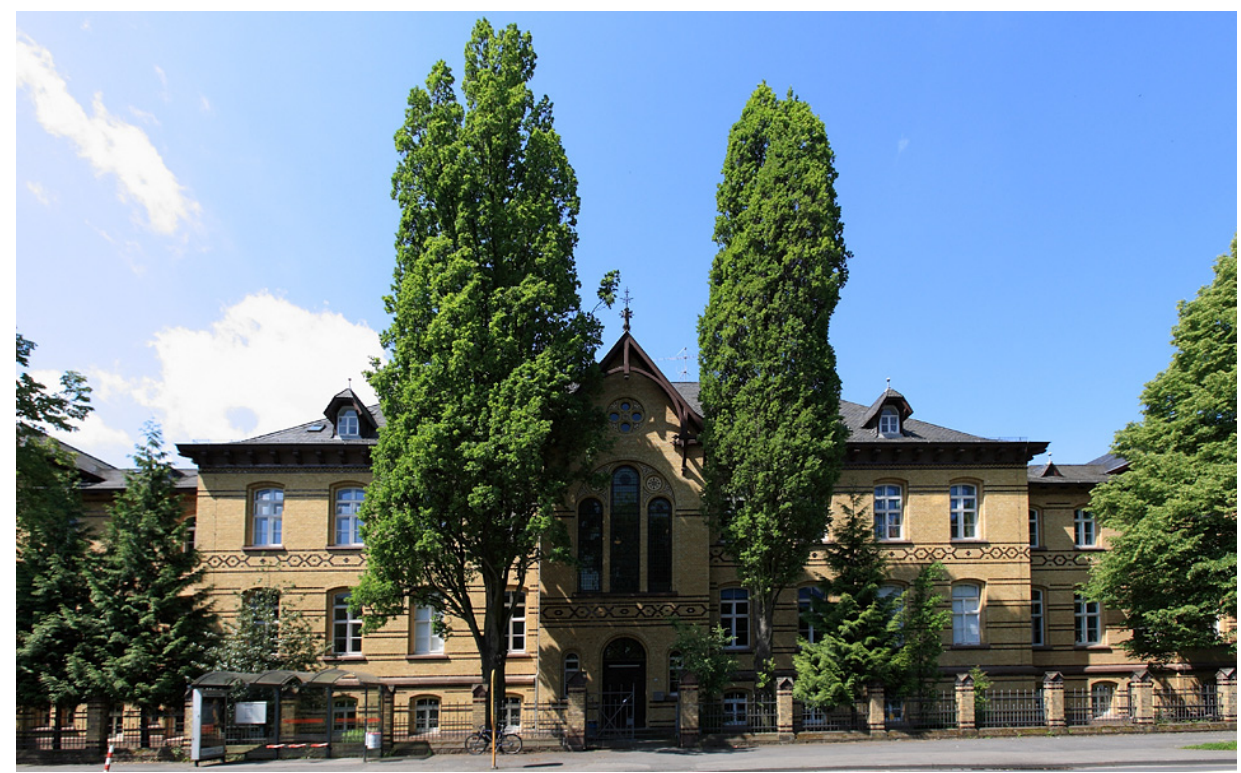

Abb. 16: Die Medizinische Klinik (1891-1977)

(Ostansicht des Mittelbaus)

Aufnahme 2009 (heute: Humboldtalle 11) 


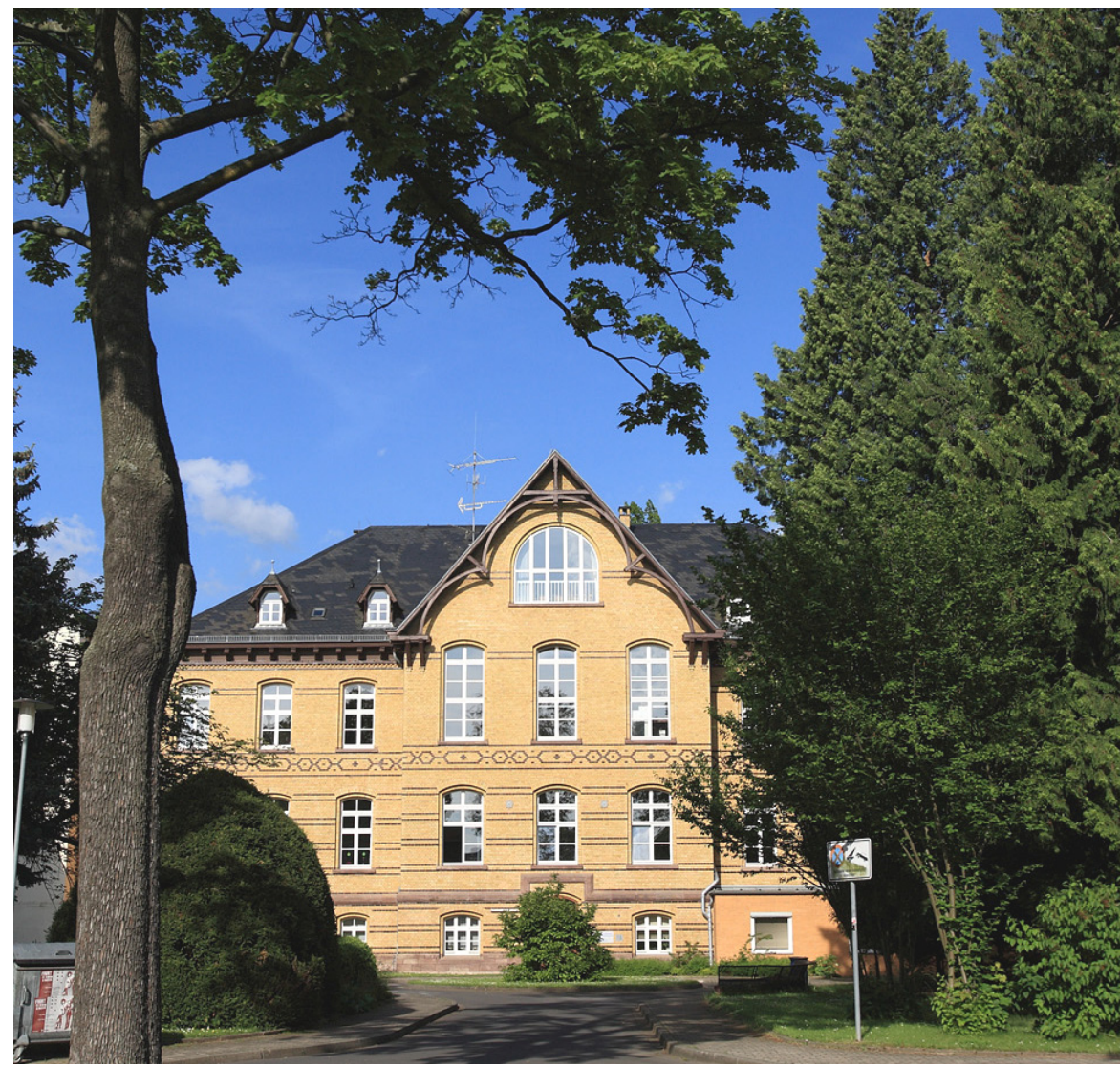

Abb. 17: Die Medizinische Klinik (1891-1977)

(Westansicht des Mittelbaus)

Aufnahme 2009 (heute: Humboldtallee 11)

Neben den spezifischen Funktionsräumen gab es ein Auditorium, das in ansteigender Form über 80 Plätze verfügte. Für den Unterricht in einigen Spezialfächern wie Laryngoskopie und Elektrodiagnostik und -therapie standen eigene Räume zur Verfügung. ${ }^{123}$

\section{Absonderungsbaracken}

Sowohl für die chirurgische als auch für die medizinische Klinik wurde in unmittelbarer Nähe je eine Absonderungsbaracke für Patienten mit ansteckenden Krankheiten errichtet. Sie lagen in einer kleinen Gartenanlage und waren durch ein zwei Meter hohes Drahtgitter nach außen abgeschlossen. 


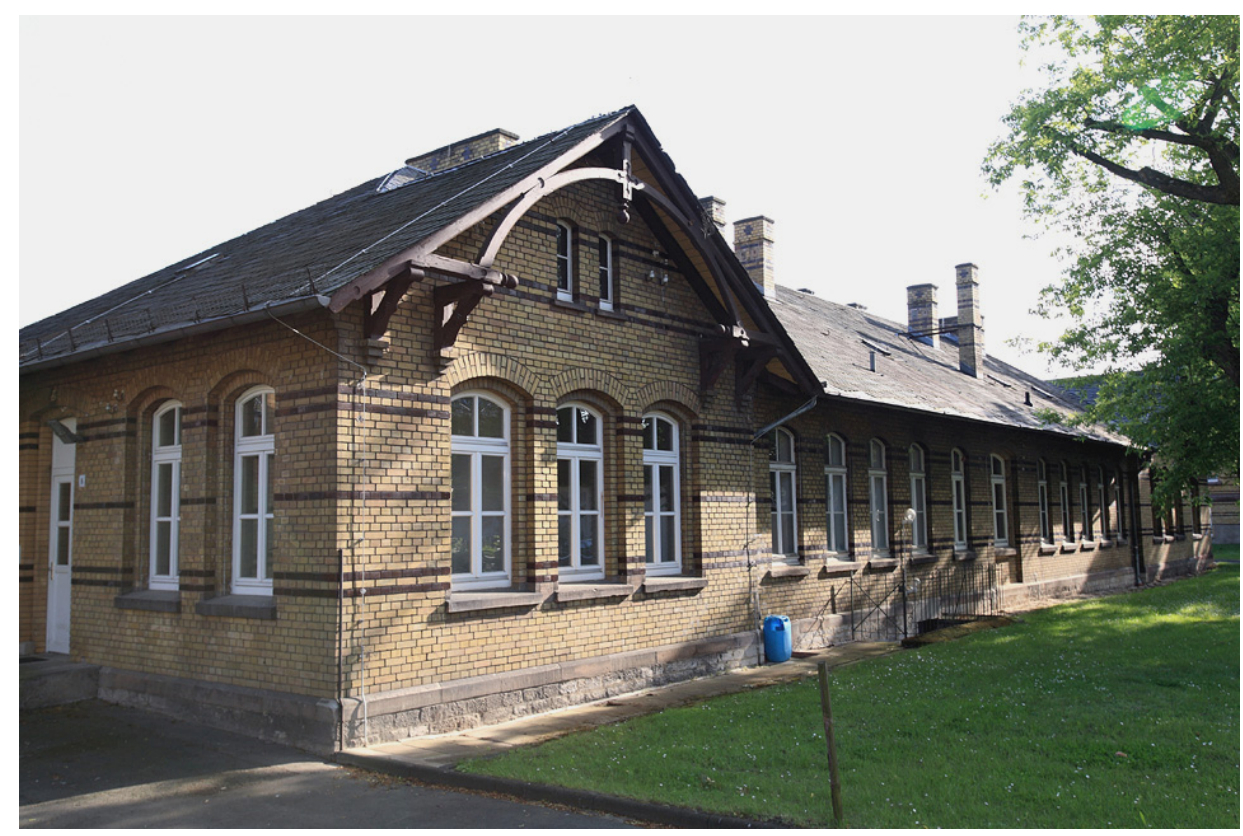

Abb. 18: Die ehemalige Absonderungsbaracke Aufnahme 2009 (heute: Käte-Hamburger-Weg 8)

\section{Die Entwicklung der Frauenklinik}

Als 1896 die Frauenklinik als dritte Teilklinik auf dem Terrain am Kirchweg eröffnet wurde, blickte sie bereits auf eine lange Tradition zurück. 1751 war sie als die erste Krankenanstalt der damals noch jungen Georg-August-Universität eröffnet worden. Ihr Aufgabengebiet war zu jenem Zeitpunkt freilich noch recht eingeschränkt und erstreckte sich im Wesentlichen auf die Geburtshilfe.

\section{Armenhospital St. Crucis}

Das St. Crucis-Hospital war nicht nur die erste klinische Einrichtung an der Göttinger Universität, sondern überhaupt die erste Geburtsklinik an einer Universität. Entgegen den damals üblichen Gepflogenheiten diente dieses Gebärhaus nicht allein der Hebammenausbildung, sondern auch der der Studenten und Ärzte. Die Initiative dazu ging von Albrecht Haller aus; Haller wollte, dass dieses Krankenhaus nicht nur eine Hebammenlehranstalt sei, sondern eine Einrichtung der Universität, dessen Leiter kein Hebammenmeister, sondern ein Professor der Geburtshilfe sei. ${ }^{124}$ 


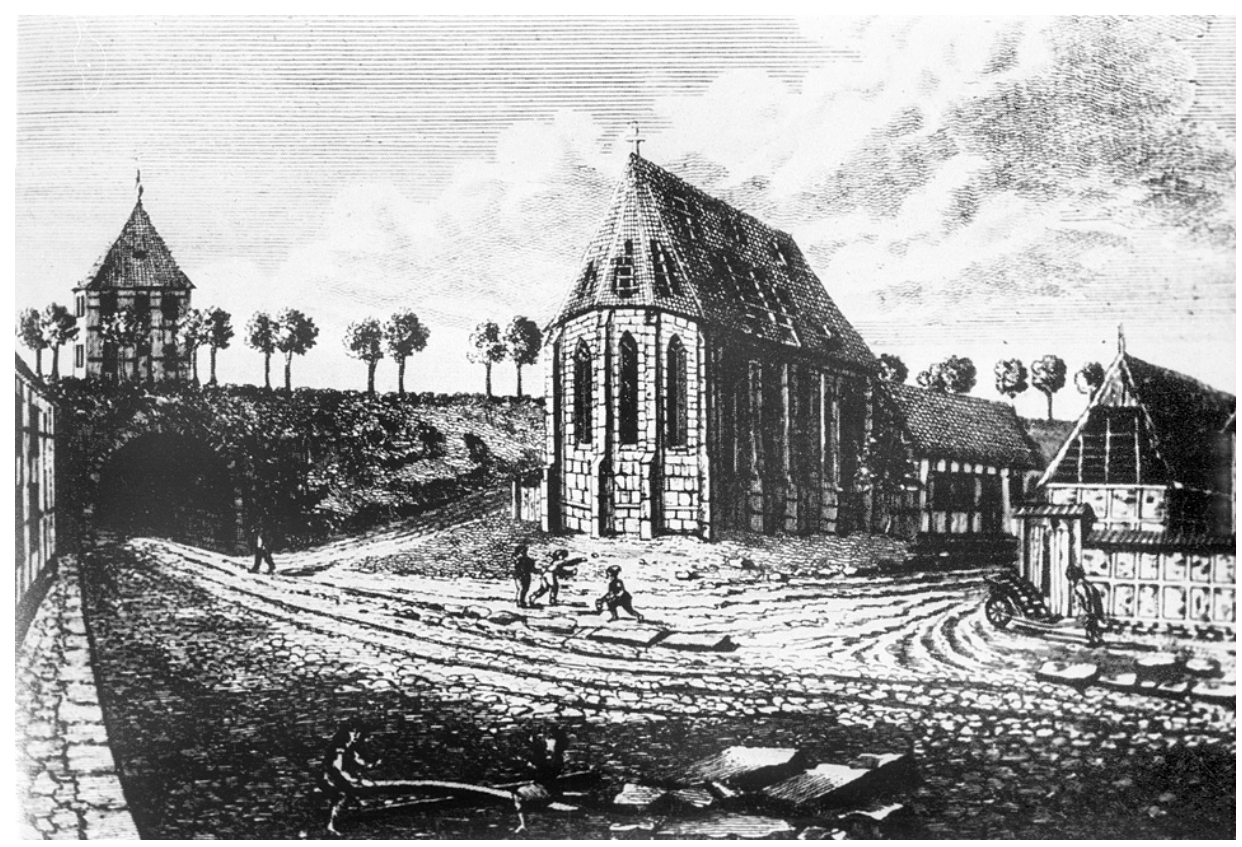

Abb. 19: „Kirche St. Crucis mit dem alten Accouchirhospitale am Geismar Thor“ Kupferstich von Christian Andreas Besemann, 1809

Als Unterkunft stellte die Stadt Göttingen das Armenhospital neben der Kirche St. Crucis in der Nähe des Geismartors zur Verfügung und übernahm auch den Umbau des sich in einem recht desolaten Zustand befindenden Hauses. „Das heisst: es wurde so zugerichtet, dass Gebährende vor Nässe und Kälte sicher darin wohnen konnten, und eine Lagerstatt, ein Friedisches Geburtsbett, einen Tisch und Stüble darin antrafen. " 125 Neben dem Entbindungszimmer besaß das Fachwerkhaus noch eine Stube und eine Kammer für die Wärterin und die Schwangeren. In räumlicher und organisatorischer Hinsicht war die Hebammenanstalt des Straßburger Bürgerhospitals, die von Johann Jacob Fried (1689-1769) geleitet wurde, Vorbild. Dieses hatte zwar keinen Universitätsstatus, aber es diente bereits seit 1734 für die Studenten als Ausbildungsstätte in der Geburtshilfe. ${ }^{126}$ Durch die Einbindung des geburtshilflichen Unterrichts in den Lehrbetrieb der Medizinischen Fakultät konnten zwei Drittel der Unterhaltungskosten für das Accouchierhaus finanziert werden. Der Rest kam von der Regierung.

Auf Anraten Hallers wurde bereits im Gründungsjahr Johann Georg Roederer (1726-1763) zum Professor der Geburtshilfe und Anatomie berufen und mit der Leitung des Gebärhospitals beauftragt. 


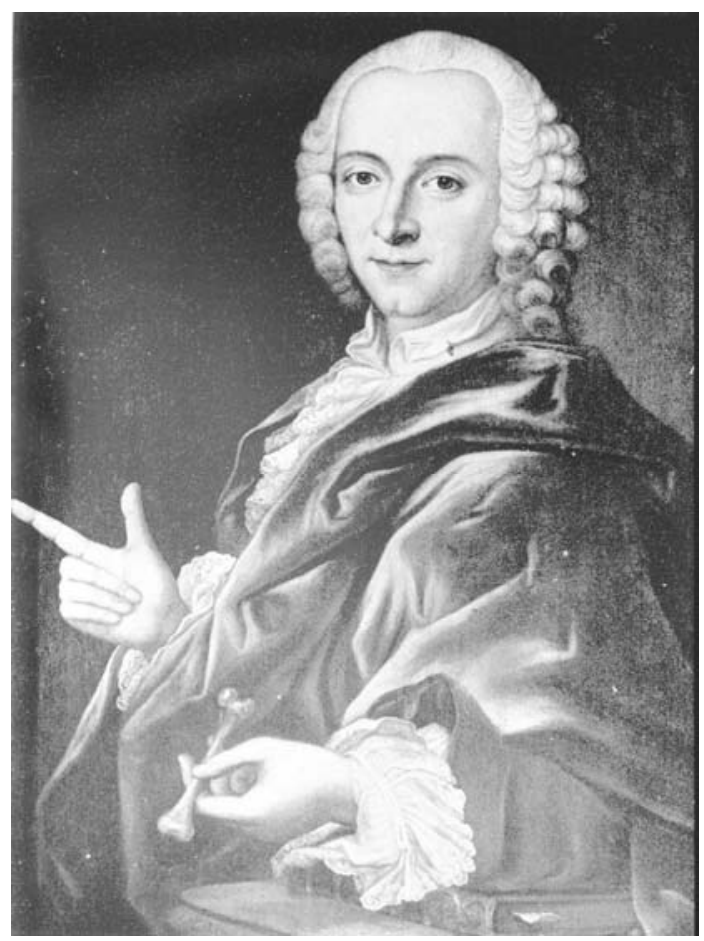

Abb. 20: Johann Georg Roederer

Öl auf Leinwand von J. W. Hauwiller, 1751

Roederer hatte in Straßburg Medizin studiert und wurde 1750 promoviert. Mit seinem Lehrbuch „Elementa artis obstetriciae in usum praelectionum academicarum “ (1753) trug er entschieden dazu bei, die Geburtshilfe auf eine wissenschaftliche Grundlage zu stellen. Noch heute erinnert die „Roederer-Kopfeinstellung“, die die maximal mögliche Verkleinerung des kindlichen Kopfes beim Geburtsvorgang bezeichnet, an sein Wirken. Roederer war sowohl für den medizinischen als auch für den wirtschaftlichen Bereich zuständig. Unterstützt wurde er dabei von einem aus dem Kreis der Studenten ausgewählten „Ökonomus“. Dieser führte Buch über Einnahmen und Ausgaben, machte die täglichen Besuche bei den Wöchnerinnen und leitete bei Abwesenheit des Professors die Geburt. Des Weiteren war eine Wärterin angestellt, die für Sauberkeit und Pflege zu sorgen hatte.

Im Sommersemester 1752 begann dann der Lehr- und Ausbildungsbetrieb. Zu Beginn litt der Unterricht unter der zu geringen Anzahl von Patientinnen. Da zu jener Zeit keine Frau zur Entbindung in ein Hospital ging, vor allem nicht in ein solches, in dem sie auch noch zu Ausbildungszwecken dienen sollte, waren es zunächst nur arme, meist unverheiratete Schwangere, die im Accouchierhaus ent- 
banden. Doch auch sie kamen meistens nur auf Grund von rechtlichen, finanziellen und sozialen Vorteilen. So wurde ihnen für ihren „Fehltritt“ gerichtliche Straffreiheit zugesichert und die Kirchenbuße erlassen. Jede Schwangere, die sich zur ambulanten Untersuchung im St. Crucis-Hospital vorstellte, erhielt dafür wöchentlich sechs Groschen und während ihres Aufenthalts im Hospital neben kostenfreien Medikamenten, 14 Groschen für die Verpflegung, 18 Groschen zur Taufe des Kindes und als Abschiedsgeschenk weitere 18 Groschen. War das Kind gestorben, so zahlte das Hospital 24 Groschen für das Begräbnis. Sogar ein „Kopfgeld" wurde ausgesetzt; für jede Schwangere, die im Accouchierhaus ihr Kind zur Welt brachte, wurden dem Vermittler neun Groschen bezahlt. ${ }^{127}$

Unter diesen Voraussetzungen kamen während Roederers Leitung von Dezember 1751 bis Anfang April 1763 insgesamt 232 Kinder zur Welt. Über seine Tätigkeit legte er akribisch Rechenschaft ab und erfasste in Tabellen die Zahl der Geburten, die aufgetretenen Schwierigkeiten und das dabei eingeschlagene Vorgehen sowie die Höhe der im Hospital unterrichteten Studenten. ${ }^{28}$ Nach Roederers überraschendem Tod leiteten zunächst die Anatomen Heinrich August Wrisberg und Philipp Georg Schröder die Entbindungsanstalt, ehe dann 1765 Wrisberg neben seinen Aufgaben in der Anatomie offiziell zum Leiter bestellt wurde. Während seiner Amtszeit in den Jahren 1765 bis 1784 sank die Geburtenzahl auf insgesamt 576. ${ }^{129}$

\section{Interimslösung bei der Marienkirche}

Bereits Roederer hatte wegen der eingeschränkten Räumlichkeiten, denn mehr als sechs Schwangere konnte das St. Crucis-Hospital nicht aufnehmen, nach Verbesserungen gestrebt. Doch verhinderte zunächst der Siebenjährige Krieg entsprechende Überlegungen, die aber dann ab 1782 konkretisiert wurden. Es sollte ein eigenes zweckmäßiges Gebäude errichtet werden, ,das zugleich der Stadt und Universität zur Zierde gereichen könnte. "130

1785 wurde Johann Heinrich Fischer (1759-1814) Leiter der Entbindungsanstalt. Er hatte in Würzburg, Erlangen und Göttingen studiert; hier wurde er 1781 promoviert, 1782 außerordentlicher Professor und 1786 ordentlicher Professor. Unter seiner Leitung wurde das inzwischen völlig baufällig gewordene St. CrucisHospital abgerissen und an seiner Stelle ein Neubau für das Accouchierhaus errichtet, dessen feierliche Grundsteinlegung am 17. September 1785 begangen wurde. Während der knapp fünfjährigen Bauzeit wurde als Interimslösung die zweite Etage im Hause des Lohgerbermeister Dege bei der Marienkirche angemietet. Das war in räumlicher Hinsicht ein gewisser Fortschritt. Es standen nun vier Stuben, drei Kammern, eine Küche und Nebenräume zur Verfügung. Bis zum Dezember 1787 entbanden dort 81 Frauen, was durchschnittlich einen leichten Zuwachs ausmachte. Neben den Studenten wurden auch mehrere Frauen zu Hebammen ausgebildet. Mit der Eröffnung des neuen Accouchier-Hospitals im März 1791 wurde die Interimslösung wieder aufgegeben. ${ }^{131}$ 


\section{Accouchier-Hospital}

Die neue „Königliche Entbindungsanstalt“, von Georg Christoph Lichtenberg (1742-1799) als ,Akekouchier - Palast" verspottet, ${ }^{132}$ stellte innerhalb der damaligen Krankenhausentwicklung einen gewaltigen Fortschritt dar. Sie entsprach in allen Belangen den Ansprüchen einer modernen Entbindungsklinik. Das Gebäude mit seinem zurückversetzten Mittelteil hatte auf der Vorder- und Rückseite Tore, die ein problemloses Hinein- und Hinausfahren mit der Kutsche ermöglichten. Eine kuppelartige, mit Säulen umrahmte Öffnung dominierte das Innere, an dessen Seite sich eine Treppe befand. Abgesehen von der eindrucksvollen Ästhetik ${ }^{133}$ erfüllte diese Konstruktion auch den Zweck, dem Gebäude ausreichend Licht und frische Luft zuzuführen.

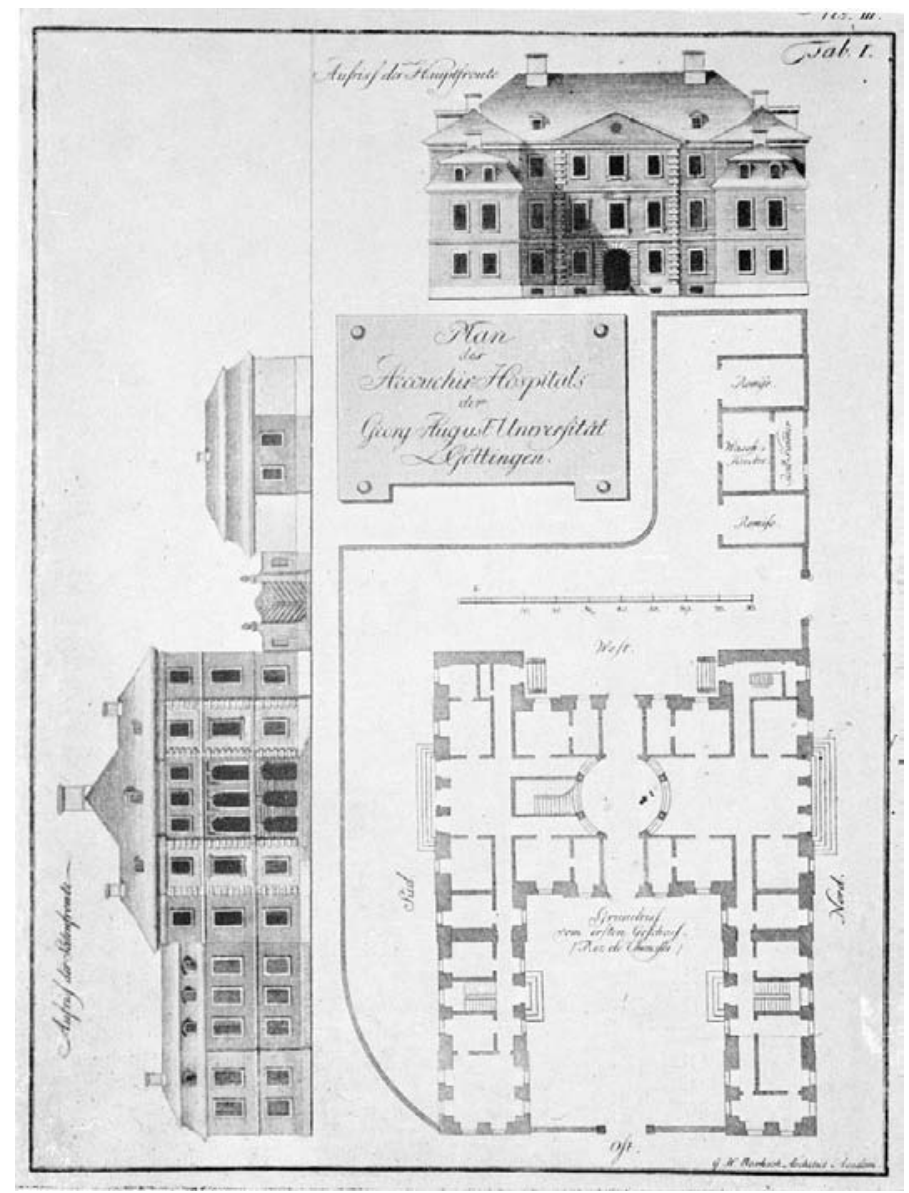

Abb. 22: "Plan des Accouchir-Hospitals"

Kupferstich aus: Pütter II (1788), Anhang No. III. Tab. I. 


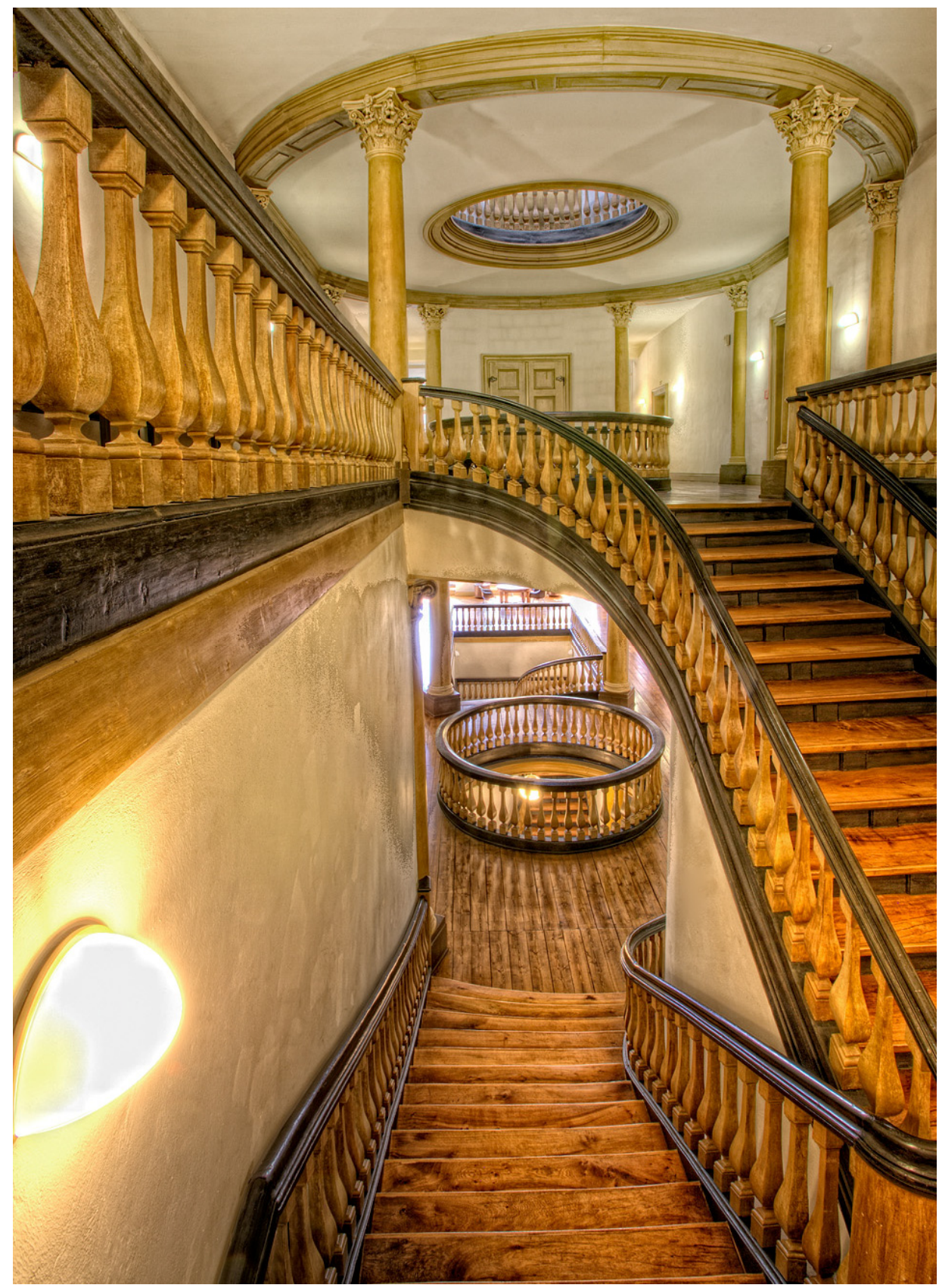

Abb. 21 : Das „sonderbar erbaute“Treppenhaus des Accouchier-Hospitals Aufnahme 2009 (heute: Kurze Geismarstraße 1) 
Wirtschaftsbereich, Pflege und Versorgung der Schwangeren, Unterricht sowie die Wohnung des Direktors waren auf die drei Etagen verteilt und sauber voneinander getrennt. Das Erdgeschoss war in erster Linie den Bedürfnissen der Haushaltung vorbehalten. Neben der Wohnung des Verwalters gab es auf dieser Etage noch je zwei Stuben und Kammern für Hebammenschüler, die im Hospital ausgebildet wurden und einen Raum, der für die Sektion der hier Verstorbenen vorbehalten war.

Entbindungs- und Hörsaal lagen im mittleren Geschoss zusammen mit sechs beheizbaren Zimmern, zwei Kammern und einer Wohnstube für die Hebamme. Die Zimmer der Schwangeren und Wöchnerinnen waren jeweils mit zwei Betten und zwei kleineren Kindbettstellen ausgestattet. Jedes Zimmer hatte ein Thermometer zur Überwachung der Raumtemperatur und ein hohes Fenster mit Vorhang. Am oberen Flügel war ein Blechrohr angebracht, das schräg nach oben zur Decke hin verlief und durch das beständig frische Luft ins Zimmer fließen konnte. Neben dem Hörsaal befanden sich die Registratur und ein Raum für die Sammlung anatomischer Präparate.

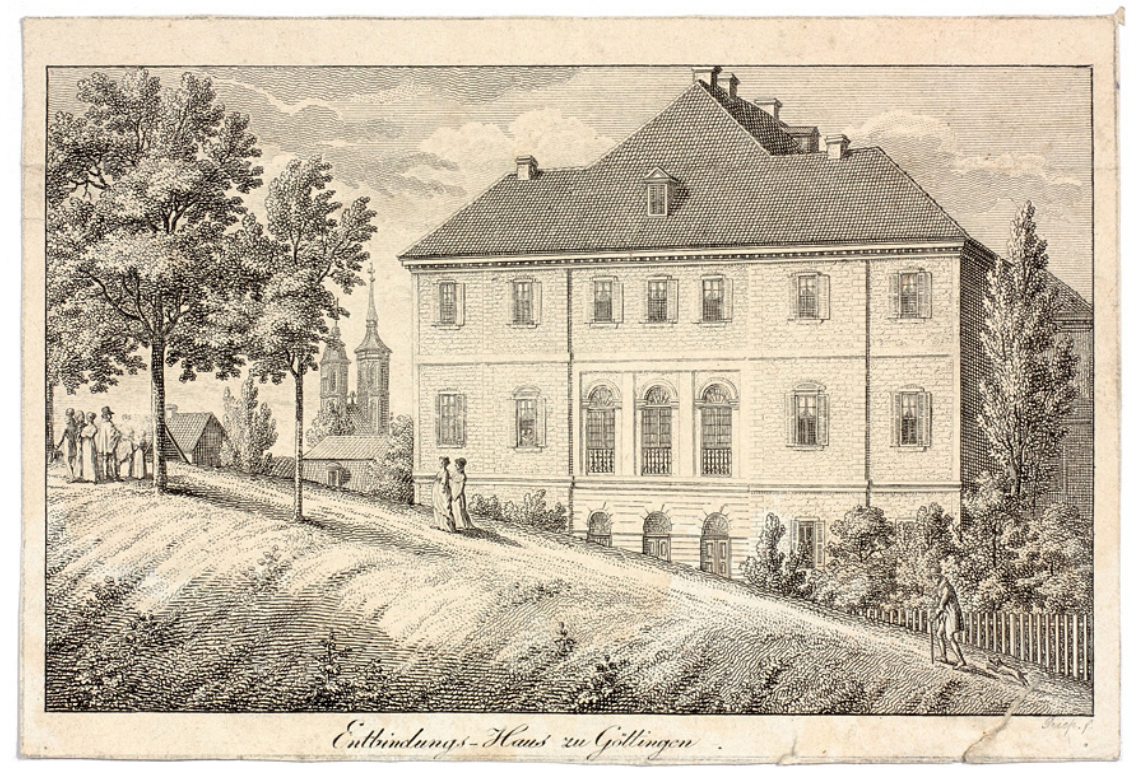

Abb. 23: Das Accouchier-Hospital

Blick über den Wall auf die Südfront des Gebäudes

Kolorierte Radierung von Christian Andreas Besemann, 1811

Im Obergeschoss waren die Wohnung des Hospitaldirektors und zwei bestens ausgestattete Zimmer, in denen Damen höheren Standes gegen Bezahlung der 
Verpflegung, Miete und sonstiger Bedürfnisse unerkannt und in aller Stille ihr Wochenbett halten konnten. ${ }^{134}$

Nicht nur das Gebäude selbst mit seinen Funktionen, sondern die gesamte Hospitalanlage war wohl durchdacht. An der Südseite der Stadt, unmittelbar am Wall gelegen, stand es ganz frei ohne angrenzende Häuser und bot so durch die das Hospital umgebenden Bäume, Büsche und Gärten sowie den Blick auf die Stadt nach allen Seiten eine angenehme Abwechslung. Vor dem Haus an der Hauptseite war ein kleiner Garten, auf der anderen Seite ein Hof mit Brunnen und weiteren Nebengebäuden.

Aufnahme fand jede verheiratete oder unverheiratete Schwangere ohne Rücksicht auf Heimat und Religion. Allerdings war die Zahl aus Kapazitätsgründen auf 16 Schwangere beschränkt. Während ihres Aufenthalts, der sich in der Regel auf vier bis sechs Wochen vor, im Sommer zwei und im Winter drei Wochen nach der Geburt erstreckte, leisteten die Wöchnerinnen leichte Arbeiten, die ihnen bei der Entlassung vergütet wurden. Kinder verstorbener Mütter wurden im Notfalle auf Kosten des Hospitals erzogen. Insgesamt war die Sterblichkeit aber auffallend gering. ${ }^{135}$

Nachdem Fischer im April 1792 als Leibarzt zum Fürsten nach NassauWeilheim ging, wurde Friedrich Benjamin Osiander (1759-1822) im Oktober als ordentlicher Professor der Medizin und Entbindungskunst berufen und mit der Leitung des Accouchier-Hospitals betraut. Er hatte in Tübingen studiert, wurde 1779 promoviert und erwarb sich darüber hinaus vor allem bei Johann Jacob Fried in Straßburg und Georg Wilhelm Stein (1737-1803) in Kassel umfassende Kenntnisse in der Geburtshilfe. Der Schwerpunkt seines umfangreichen wissenschaftlichen Werkes lag eindeutig auf der Entbindungskunst, zu der er u.a. ein dreibändiges „Lehrbuch“(1799-1825), einen zweibändigen „Grundriß“ (1802) und ein „Handbuch“(1818-1825) verfasste. ${ }^{136}$

Mit Osiander und der neuen Geburtsklinik waren nun ideale Voraussetzungen geschaffen für einen weiteren Höhepunkt in der Geschichte der Medizinischen Fakultät. Obwohl Osiander zunächst auf nahezu allen Gebieten der Medizin tätig war, nahm die Geburtshilfe und hier insbesondere der operative Teil des Fachs immer mehr die zentrale Stellung in seinem medizinischen Wirken ein. Die Geburtshilfe verstand er als Entbindungskunst, deren Aufgabe es war, der Gebärenden bei der natürlichen Geburt die größtmögliche Bequemlichkeit und Erleichterung zu verschaffen und bei einer widernatürlichen Geburt Gesundheit, Leben und Integrität von Mutter und Leibesfrucht in höchstem Maße zu bewahren.

Im Mittelpunkt seiner mehr aktiven Geburtshilfe stand die Verwendung der Geburtszange. Mit ihr, die er seine ,künstlichen Hände" nannte und die nach seiner geburtsphysiologischen Vorstellung und Erfahrung konstruiert war, sollte eine widernatürliche Geburt in eine natürliche verwandelt werden. ${ }^{137}$

Während seiner Göttinger Tätigkeit wandte er die Zange bei 2.540 Geburten 1.016 mal an. ${ }^{138}$ Osiander trug auch selbst durch eigene Erfindungen zur Entwick- 
lung der Geburtszange bei. Sie bildet in ihren verschiedenen Varianten das Kernstück einer bedeutenden, weitgehend von Osiander zusammengetragenen Sammlung aus dem klinischen Alltag der Frauenheilkunde, dem „Museum Anatomicum Osiandrium "139 Bis zu seinem Tod am 23. März 1822 leitete Friedrich Benjamin Osiander die Entbindungsklinik. Seine Nachfolger erreichten nicht mehr den Glanz seines breiten Wirkens. Erwähnt werden sollte Eduard Carl Caspar Jacob von Siebold (1801-1861), der aus der bekannten „Asklepiadenfamilie der Siebolde“ stammte und das Accouchier-Haus zwischen 1833 und 1861 leitete.

Seine Tätigkeit fiel in die Blüte der Göttinger Universität, die durch Persönlichkeiten wie Carl Friedrich Gauss (1777-1855), Wilhelm Weber (1804-1891), Friedrich Wöhler (1800-1882) und Jacob Henle geprägt wurde, aber auch durch Künstler wie Joseph Joachim (1831-1907) und Johannes Brahms (1833-1897), dessen Romanze mit Siebolds Tochter Agathe die Gemüter erregte. ${ }^{140}$

Von Siebold studierte in Berlin und Göttingen, wurde 1826 in Berlin promoviert und habilitierte sich 1827 für das Fach Geburtshilfe. 1829 wurde er Professor in Marburg und 1831 in Göttingen. Da er in seinem Denken weitgehend naturphilosophischen Konzepten verhaftet war, stand er der naturwissenschaftlich fundierten Medizin des 19. Jahrhunderts eher distanziert gegenüber und vertrat auch bei der Entbindungskunst eine eher konservative Haltung. Neben der spezifisch wissenschaftlichen Tätigkeit, bei der Werke wie ,Anleitung zum geburtshülflichen technischen Verfahren am Phantome“ (1828), der zweibändige „Versuch einer Geschichte der Geburtshülfe“ (1839-1845) und das „Lehrbuch der gerichtlichen Medicin“ (1847) entstanden, beschäftigte sich von Siebold als humanistisch gebildeter Arzt auch mit Themen der klassischen Philologie und übertrug beispielsweise Satiren Juvenals.

Auch in der Geburtshilfe fanden die durch die naturwissenschaftlich fundierte Medizin des 19. Jahrhunderts erzielten Erkenntnisse den entsprechenden Widerhall. Neben quantitativen Veränderungen, wie der Zunahme der klinischen Entbindungen und der studentischen Hörerzahlen, wurden Charakter und Struktur der tradierten Entbindungskunst entscheidend durch die allmähliche Einbeziehung der gesamten Gynäkologie in das Arbeitsprogramm des Geburtshelfers mit allen daraus folgenden Aufgaben für die klinische Praxis, Lehre und Forschung beeinflusst. Die systematische Erfassung der normalen und pathologischen Physiologie der Frau führte zu neuen Sichtweisen und zur Erweiterung des Spezialfachs. Aus der einstigen Hebammenkunst war die Frauenheilkunde, aus dem Geburtshelfer der Frauenarzt geworden. ${ }^{141}$ Eine solche Entwicklung stellte naturgemäß auch an die herkömmliche Geburtsklinik neue gravierende Anforderungen. Die aufkommende operative Gynäkologie, unterstützt durch Kenntnisse zur Asepsis und neue Narkosetechniken, erforderte neue Operationssäle, die Umsetzung neuer Laboratoriumsmethoden verlangte entsprechende räumliche und materielle Voraussetzungen, die das Accouchier-Hospital nicht mehr erfüllen konnte. 


\section{Frauenklinik an der Humboldtallee}

Unter Heinrich Max Runge (1849-1909), der seit 1888 die Geburtsklinik leitete, gelang es nach jahrelangen Verhandlungen, einen Neubau in Angriff zu nehmen. 1894 wurde der Grundstein gelegt und am 1. November 1896 konnte die Klinik feierlich eröffnet werden. Die gesamte Anlage der neuen Frauenklinik bestand aus vier eigenständigen Gebäuden. Neben dem dreigeschossigen Hauptbau gab es auf der einen Seite ein sogenanntes Neben- und ein Waschhaus und auf der anderen das Direktoren-Wohnhaus. Zwischen 1928 und 1931 wurden Hauptbau und Nebenhaus durch Anbauten erweitert.

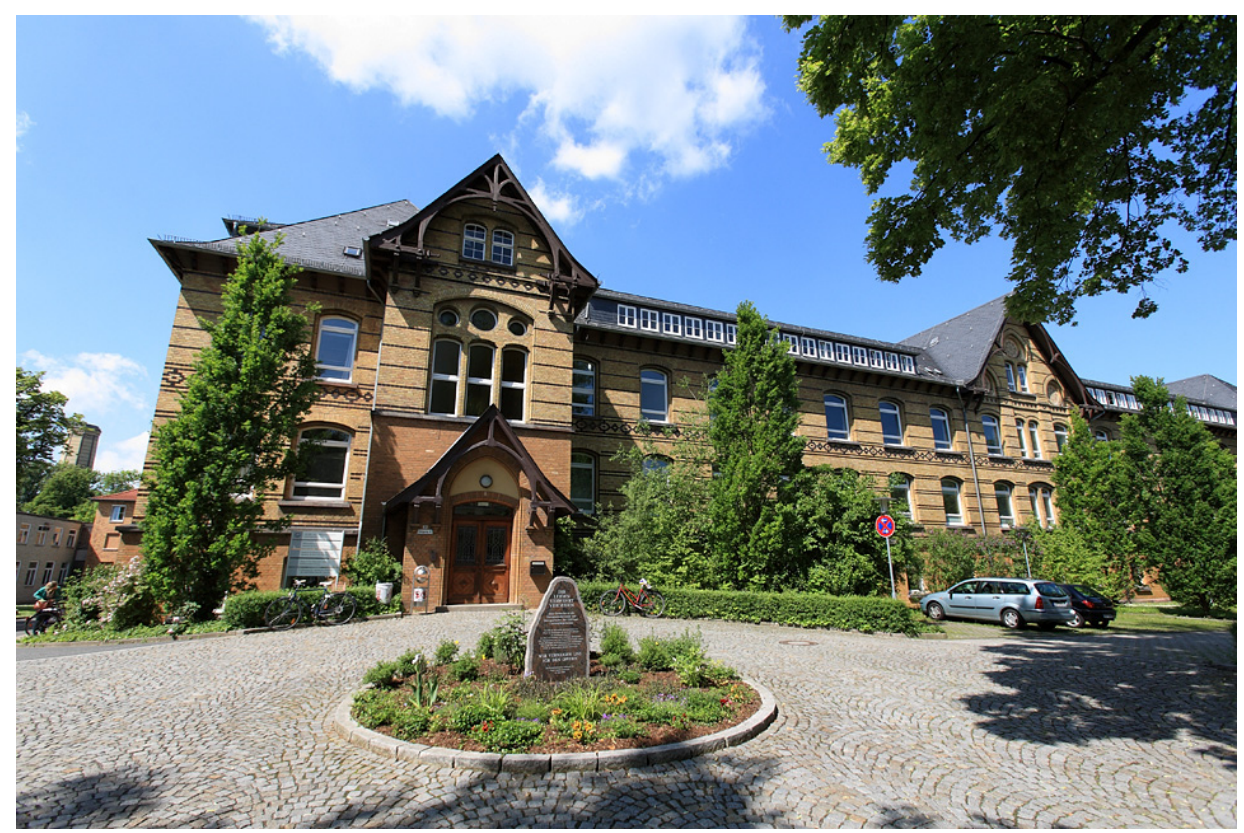

Abb. 24: Die Frauenklinik (1896-1988)

(Ostansicht des Hauptgebäudes) mit dem 2008 errichteten Zwangsarbeiterdenkmal Aufnahme 2009 (heute: Humboldtallee 19)

Haupt- und Nebenbau waren mit insgesamt 116 Betten ausgestattet. Dieser Umfang galt als Optimum. Zum einen boten sich dadurch genügend Krankheitsfälle für den Unterricht, zum anderen war eine eingehende und persönliche Untersuchung und Behandlung der einzelnen Patienten gewährleistet. Die Raumanordnung zeichnete sich durch größte Übersichtlichkeit und kurze Wege aus. Die wesentlichen Einrichtungen im Erdgeschoss waren die Poliklinik, die Röntgendiagnostik und -therapie sowie die Räume für die Hausschwangeren. 
Im ersten Obergeschoss war der Lehrbereich mit einem großen Hörsaal für 110 Plätze, mehreren Übungsräumen und einem Vorbereitungsraum, der auch für Operationen genutzt werden konnte. Auf der gleichen Etage befanden sich die geburtshilfliche Abteilung mit zwei Kreißsälen und die Zimmer für die Wöchnerinnen und deren Kinder.

Im zweiten Obergeschoss waren wissenschaftliche Arbeitsräume und die gynäkologische Abteilung mit Operationssaal und Krankenzimmern. Im Keller befanden sich die Heilbäderabteilung, die geburtshilfliche Sammlung und im Dachgeschoss Wohnungen. Das Nebenhaus hatte im Erdgeschoss mehrere Krankenzimmer zum Teil für infektiöse Fälle, und im Obergeschoss Ärztewohnungen. ${ }^{142}$

Wie kein anderer prägte Heinrich Martius (1885-1965) das Bild der neuen Frauenklinik, deren Direktor er von 1926 bis 1954 war. Martius hatte in Freiburg, Leipzig und Rostock studiert, wo er 1910 promoviert wurde. 1919 habilitierte er sich in Bonn und wurde 1922 außerordentlicher Professor. 1926 erhielt er den Ruf nach Göttingen. Sein wissenschaftliches Denken war von dem Bemühen gekennzeichnet, die einzelnen Spezialgebiete der Gynäkologie vor der Aufsplitterung zu bewahren. Dies dokumentiert auch sein umfangreiches Werk, das Publikationen zu nahezu allen gynäkologischen Bereichen bietet. Martius verfasste Lehrbücher und Monographien zur Geburtshilfe, zur Gynäkologie, zu geburtshilflichen und gynäkologischen Operationen sowie zur gynäkologischen Orthopädie, die alle hohe Auflagen erreichten. ${ }^{143}$

Von seinem gesellschaftlichen Engagement zeugt „Das kleine Frauenbuch“ (1956), das knapp, leicht verständlich und mit augenfälligen Abbildungen versehen, Form, Bau und Funktion des weiblichen Körpers erklärt, um Missverständnisse sowie Irrglauben zu beseitigen. ${ }^{144}$

\section{Augenklinik}

Mit der Augenklinik war das Gesamtkonzept des Klinikareals am damaligen Kirchweg abgeschlossen. Der Bau wurde im Mai 1904 begonnen und im April 1906 fertiggestellt. In seiner äußeren Gestaltung passte sich das Gebäude nahtlos in das Gesamtbild ein. Der insgesamt $53 \mathrm{~m}$ lange Baukörper hatte im nördlichen Teil einen $27 \mathrm{~m}$ langen Seitenflügel. Das Hauptgebäude besaß fünf und der Seitenflügel vier Geschosse. Die technische Ausführung entsprach im Wesentlichen den drei anderen bereits erbauten Kliniken. Lediglich das Beleuchtungssystem war auf die spezifischen Aufgaben einer Augenklinik abgestimmt. Neben der Elektrizität fand auch Gasbeleuchtung („Auerlicht") Verwendung. Alle Krankenzimmer waren mit elektrischen Lampen ausgestattet, die das Licht nach oben an die Decke warfen und es nach unten durch einen fast geschlossenen grün-weißen Glasschirm abdeckten, um Blendungen zu verhindern. Ebenfalls in therapeutischer Absicht wurden die Wände in den Zimmern bis zur Decke hellgrün gestrichen und in den 
Korridoren sowie den Treppenhäusern oberhalb eines hellgrünen Sockels in Weiß, um die Helligkeit zu intensivieren. Ferner gab es ein Haustelefon, durch das alle Stockwerke miteinander verbunden waren, und einen geräumigen Fahrstuhl, in dem der frisch operierte Patient auf dem Operationstisch in die jeweilige Abteilung gebracht werden konnte. Wirtschaftsbetrieb, Lehrbereich, Poliklinik und Krankenabteilungen waren voneinander abgetrennt.

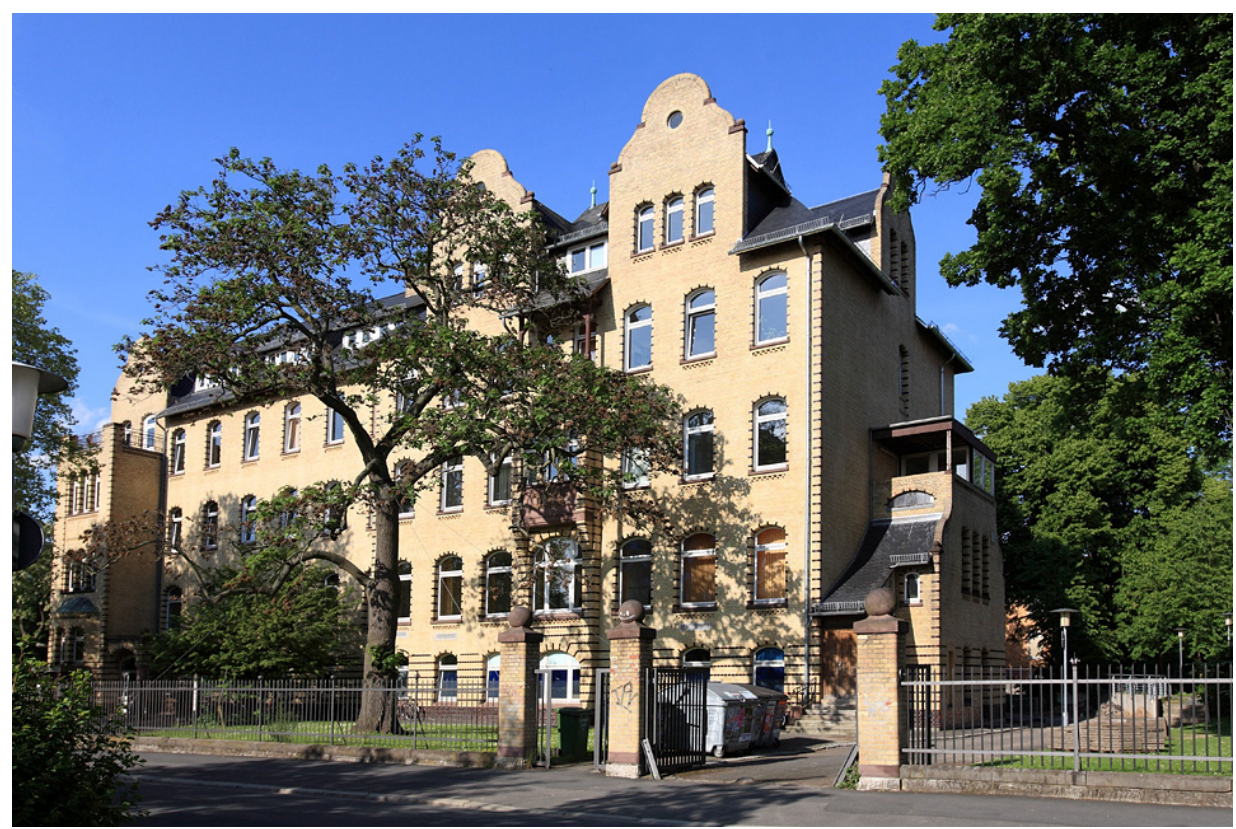

Abb. 25: Die Augenklinik (1906-1979)

(Westansicht des Hauptgebäudes)

Aufnahme 2009 (heute: Goßlerstraße 10)

Im Erdgeschoss befanden sich in erster Linie die Räume der Haushaltung; daneben gab es ein Ärztecasino und einen Raum für Tierversuche. Im ersten Stock waren Hörsaal, Arbeitsräume für die Forschung und die Poliklinik mit ihren entsprechenden Funktionsräumen. Die Frauen- und Kinderabteilung lag im zweiten Stock. Hier gab es neben dem Operationssaal, fünf Krankenzimmer mit unterschiedlicher Bettenzahl von drei bis sechs, zwei Einbettzimmer für Unruhige oder Privatpatienten und einen großen Krankensaal mit 16 Betten. Das dritte Stockwerk enthielt die Männerabteilung. Die Aufteilung der Krankenzimmer und ihre jeweilige Bettenzahl entsprach der der Frauenabteilung. Das vollständig ausgebaute Dachgeschoss beherbergte neben einigen Funktionsräumen Wohnungen für das Personal. Der damalige Leiter der Augenklinik Arthur 
von Hippel (1841-1916) legte besonderen Wert auf eine weitestmögliche Selbstständigkeit der Augenklinik. Sowohl die Verwaltung als auch der gesamte Wirtschaftsbetrieb verblieben in ihrer alleinigen Zuständigkeit. ${ }^{145}$ Damit waren nun alle zu diesem Zeitpunkt existierenden Kliniken an einem zentralen Punkt vereinigt und die ursprüngliche Überlegung, die unterschiedlichen Teilkliniken an einem Ort zusammenzuführen, war verwirklicht worden. Dies sollte bis zum Jahre 1977 Bestand haben.

\section{Kinderklinik}

Die Entwicklung der Kinderheilkunde zu einem medizinischen Spezialfach vollzog sich über einen längeren Zeitraum. Weder die Geburtshilfe noch die innere Medizin maßen dieser Thematik großes Gewicht bei und behandelten sie in den Vorlesungen recht stiefmütterlich. Erst als die Bevölkerungsstatistik gegen Ende des 19. Jahrhunderts eine alarmierend hohe Sterblichkeit vor allem der Säuglinge und damit einhergehend abnehmende Geburtenzahlen indizierte, begann ein Umdenken. Der Staat konnte nicht mehr länger unbeteiligt zusehen und sorgte für eine Verbesserung des diesbezüglichen medizinischen Unterrichts. ${ }^{146}$ Doch erst in den Nachkriegsjahren zwischen 1919 und 1924 wurde das Fach an den meisten Universitäten etabliert. So auch 1919 in Göttingen. ${ }^{147}$

Zwar hatte bereits Johann Gottfried Brendel im Wintersemester 1748/49 eine Vorlesung zum Thema „de morbis mulierum et infantum " angekündigt und in der Folgezeit widmeten sich auch Geburtshelfer wie Roederer oder Osiander sowie Privatdozenten und Assistenten der medizinischen Klinik der kinderheilkundlichen Thematik, doch eine spezielle Vorlesung gab es nicht und entsprechend gering war das Interesse der Studenten. ${ }^{148}$ Und so führte die Kinderheilkunde auch in Göttingen in jener Zeit „im Schosse der inneren Medizin ein ziemlich latentes Dasein. "149 Nicht zuletzt auch unter dem Druck der neuen Prüfungsordnung für Ärzte vom 28. Mai 1901 schuf die Medizinische Fakultät schließlich ein unbesoldetes Extraordinariat mit Lehrauftrag, auf das am 1. April 1907 Bruno Salge (1872-1924) berufen wurde. Ab dem Sommersemester hielt er Vorlesungen zur klinischen und theoretischen Kinderheilkunde. Die medizinische Klinik stellte Betten und Räume für das wissenschaftliche Arbeiten bereit. ${ }^{150}$

Unter Friedrich Göppert (1870-1927) wurde zwischen 1909 und 1911 der erste Neubau der Kinderklinik errichtet. Göppert studierte in Berlin und Heidelberg, wo er 1896 promoviert wurde. Am 1. Oktober 1909 folgte er dem Ruf nach Göttingen auf das Extraordinariat für Kinderheilkunde. 1919 wurde er zum persönlichen Ordinarius und 1923 zum planmäßigen Ordinarius ernannt. Sein breitgefächertes wissenschaftliches Werk erstreckte sich von Untersuchungen zur Genickstarre, zu unterschiedlichen Infektionskrankheiten, zur Impfproblematik bis hin zu Fragen der Erziehung, zur Rolle des Schulkinderarztes und zur Euthanasie im 
Kindesalter. Es sind vor allem klinische Arbeiten, die durch exakte Beobachtungen gekennzeichnet sind. ${ }^{151}$

Die Initiative, eine eigenständige Kinderklinik zu errichten, stammte von Wilhelm His (1863-1934), der zwischen 1906 und 1907 als Direktor die medizinische Klinik leitete. Ende 1906 schlug der damalige Oberbürgermeister Georg Friedrich Calsow (1857-1931) das Grundstück an der Ecke Kirchweg/Kreuzbergweg dafür vor. Der Göttinger Bürger Ernst Dreger hatte es der Stadt zusammen mit einer Geldsumme gestiftet, um darauf ein Heim für Schwache sowie für kranke Kinder zu errichten. ${ }^{152}$

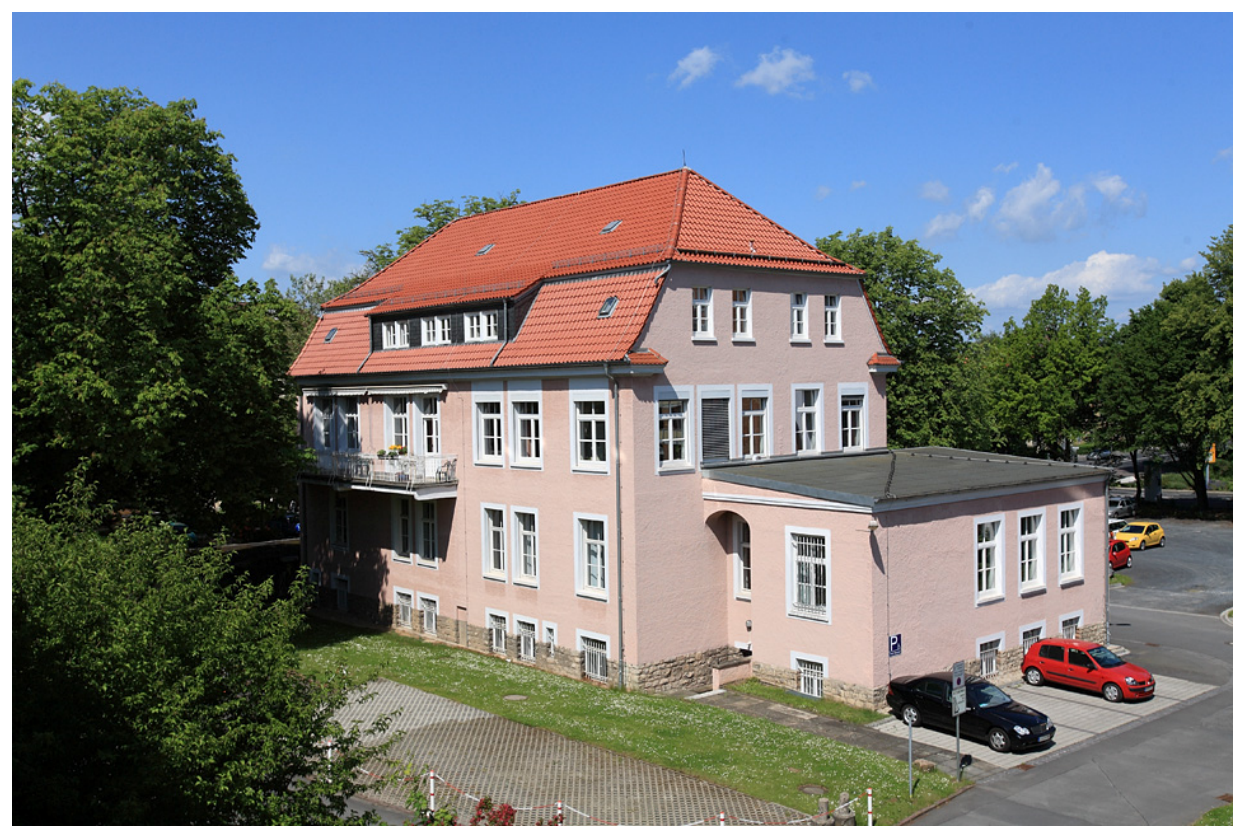

Abb. 26: Die Kinderklinik (1911-1987)

(Erstes Gebäude)

Aufnahme 2009 (heute: Humboldtallee 36)

Nach langwierigen und schwierigen Verhandlungen mit der Stadt und dem Ministerium in Berlin, bei denen die ursprüngliche Vorstellung von 30 Betten auf 20 reduziert und die Baukosten um mehr als die Hälfte gesenkt werden mussten, konnte im August 1909 mit den Bauarbeiten begonnen werden. Am 15. Januar 1911 nahm die Kinderklinik offiziell ihren Betrieb auf. 153

Hinsichtlich ihrer Kapazität konnte die klinische Abteilung bis zu 24 Kinder stationär behandeln. Im Normalfall war sie jedoch für 18 bis 19 Patienten eingerichtet. Für die Aufnahme von Mutter und Kind mit erster Klasse Verpflegung gab es zwei Einzelzimmer. In dringenden Fällen war eine Aufnahme auch für 
weniger begüterte Mütter möglich. Neben der Poliklinik mit drei gesonderten Sprechzimmern und einer Milchküche besaß das Gebäude auch zwei Laboratoriumsräume. Ferner bildete die Kinderklinik jährlich drei Mädchen aus angesehenen Familien als Säuglingspflegerinnen aus. ${ }^{154}$

Durch die erheblichen Streichungen an der ursprünglichen Konzeption war die Kinderklinik von Anfang an zu eng angelegt. Hinzu kam eine wachsende Inanspruchnahme, die die Kapazitätsgrenze des Öfteren überschritt und häufig zu Hausinfektionen führte. In einer ersten Erweiterungsstufe wurde 1915 eine Isolierbaracke mit acht Betten eröffnet. Mitte 1917 wurde mit den Planungen eines Erweiterungsbaues begonnen, den das Ministerium in Berlin Anfang 1918 zunächst jedoch ablehnte. ${ }^{155}$ Immerhin gelang es, einen eigenen Hörsaal zu errichten, der ab März 1921 benutzt werden konnte.

Im September 1920 begannen die Arbeiten am Neubau, für dessen Errichtung im Wettlauf mit der Geldentwertung eine Bauzeit von zwei Jahren veranschlagt worden war. Der zahlreichen Verzögerungen überdrüssig, wartete Göppert die offizielle Übergabe nicht mehr ab, nahm im April 1923 den Neubau in Betrieb und schrieb an den Kurator: „Durch den zu großen Zustrom aus öffentlichen Gründen nicht abweisbarer Kranker sind wir genötigt, den Neubau einstweilen in Benutzung zu nehmen. " 156 Die Klinik verfügte nun über 60 Betten, davon 20 in der Infektionsabteilung. 1925 erfolgte der Ausbau des Dachgeschosses zum Wohnraum für Schwesternschülerinnen, 1926 wurden ein Waschhaus erstellt und eine neue Röntgenanlage errichtet. ${ }^{157}$ Nach Göpperts Tod übernahm Hans Beumer (1884-1945) die Leitung der Kinderklinik. Auch er drängte auf weitere Vergrößerungen. 1933 bis 1936 wurden zwei Liegehallen und je eine Baracke für Diphtherie- und Scharlachkranke errichtet. Den 1938 begonnenen Planungen eines Neubaus machte allerdings der Krieg ein Ende. ${ }^{158}$

Unter Hans Kleinschmidt (1885-1977), der zwischen 1945 und 1954 die Leitung inne hatte, erhielt die Kinderklinik einen Erweiterungsbau mit 90 Betten. Es war der erste Nachkriegsbau an der Universität. ${ }^{159} 1955$ errichtete sein Nachfolger Gerhard Joppich (1903-1992) einen Neubau für die Infektionsabteilung. Damit war die Bautätigkeit auf dem Areal Kirchweg/Ecke Kreuzbergweg abgeschlossen und bis zum Winter 1987 verblieb die Kinderklinik dort.

\section{Hautklinik}

Das Spezialfach der Haut- und Geschlechtskrankheiten entwickelte sich aus der im 16. Jahrhundert stets aktuellen Venerologie und der seit dem 18. Jahrhundert beginnenden Dermatologie. Als äußere Krankheiten gehörten die Geschlechtskrankheiten in die Zuständigkeit der Wundärzte und innerhalb des Universitätsunterrichts wurde dieser Bereich den Dozenten mit chirurgischen Interessen überlassen. „Im 18. Jahrhundert bürgerte sich „De morbis venereis“ als Spezialkolleg vor allem für 
jüngere Universitätslehrer ein, obne daß eine besondere Qualifikation dazu oder gar keine Absicht zur Spezialisierung als Venerologe daraus bergeleitet werden kann. " 160 Die Hautkrankheiten hingegen wurden entsprechend der vorherrschenden Humoralpathologie als Dyskrasie der inneren Säfte betrachtet und daher der „inneren“ Medizin zugeordnet. Diese doch etwas strikte Aufteilung lässt sich für Göttingen nicht zwingend nachweisen. Die tradierten Vorlesungsankündigungen, Klinikberichte und wissenschaftlichen Publikationen zeigen, dass die venerischen und dermatologischen Krankheitsfälle bis zum Ende des 19. Jahrhunderts gemeinsam und zeitweise unter einem Dach behandelt wurden. ${ }^{161}$ Erst bei den Neubauten am Kirchweg wurde für die chirurgische und medizinische Klinik jeweils eine eigene Absonderungsbaracke errichtet, ohne dass bei der Aufnahme zwischen venerischen und dermatologischen Krankheiten differenziert wurde. ${ }^{162}$

Nachdem seit 1903 mehrere Versuche gescheitert waren, gelang es schließlich - bedingt auch durch den deutlichen Anstieg venerischer Krankheiten im Ersten Weltkrieg - im Jahre 1917 die Zustimmung des Ministerium zur Errichtung einer dermatologischen Klinik zu erhalten. Am 1. Oktober wurde Erhard Riecke (18691939) als Extraordinarius berufen und zum Direktor der neu einzurichtenden Poliklinik für Haut- und Geschlechtskrankheiten ernannt. Als Heimstätte diente die alte Absonderungsbaracke der medizinischen Klinik. Für die Poliklinik war ein einziges Zimmer mit Warteraum auf dem Gang vorgesehen. Die Frauenabteilung bestand aus zwei Zimmern mit jeweils acht Betten, die allerdings des öfteren mit bis zu 20 Betten überfüllt waren. Die Männerstation war im Erdgeschoss des Südflügels der medizinischen Klinik ausgelagert; dort waren 20 Betten vorhanden. Insgesamt waren Ausstattung und hygienische Verhältnisse mehr als dürftig. 1921 konnten durch einen Anbau die größten Übelstände abgestellt werden. Für den Unterricht und die Poliklinik war nun mehr Platz vorhanden und die hygienischen Verhältnisse konnten dadurch deutlich verbessert werden. Der Hörsaal mit 80 Plätzen war nach modernen Gesichtspunkten angelegt. Die Poliklinik hatte vier Untersuchungszimmer, ein kleines Operationszimmer, ein Röntgenzimmer und ein Zimmer für die Sammlung. Die beiden Wartezimmer waren nach Geschlechtern getrennt. Die Krankenzimmer hingegen blieben in ihrer Primitivität weiterhin bestehen. ${ }^{163}$

\section{Die Hautklinik am Steinsgraben}

Zum Jahresbeginn 1928 konnte das vollständig renovierte frühere Militärlazarett am Steinsgraben 19 als moderne Hautklinik eröffnet werden. Dies bedeutete in jeder Hinsicht eine deutliche Verbesserung. Einziger Nachteil war die räumliche Trennung zur Poliklinik, die in der Absonderungsbaracke verblieb. Auf dem parkähnlichen Gelände, das nach außen durch Büsche nahezu hermetisch abgeschlossen war, standen neben einem Wirtschafts- und Verwaltungsgebäude drei unterschiedlich lange einstöckige Pavillons. Der erste, der als Männerstation diente, 
besaß zwei große Krankensäle mit jeweils 24 Betten und zwei Einzelzimmer. Die eine Hälfte gehörte zu der Abteilung für Hautkranke, die andere der für Geschlechtskranke. Neben den üblichen Wirtschafts- und Funktionsräumen gab es einen Operationsraum und je ein Zimmer für Höhensonne und Röntgen. Im zweiten etwas kürzeren Pavillon war die Frauenstation. Analog zum ersten gab es zwei Krankensäle mit jeweils 12 Betten und drei Einzelzimmer. Auch hier waren die Räumlichkeiten zwischen den beiden Fachrichtungen unterteilt. Der dritte und kleinste Pavillon beherbergte die Privatstation mit zwei Einzel- und zwei Zweibettzimmern. Im zweistöckigen Verwaltungsgebäude befanden sich das Dienstzimmer des Direktors, drei Labore und mehrere Funktionszimmer. ${ }^{164}$

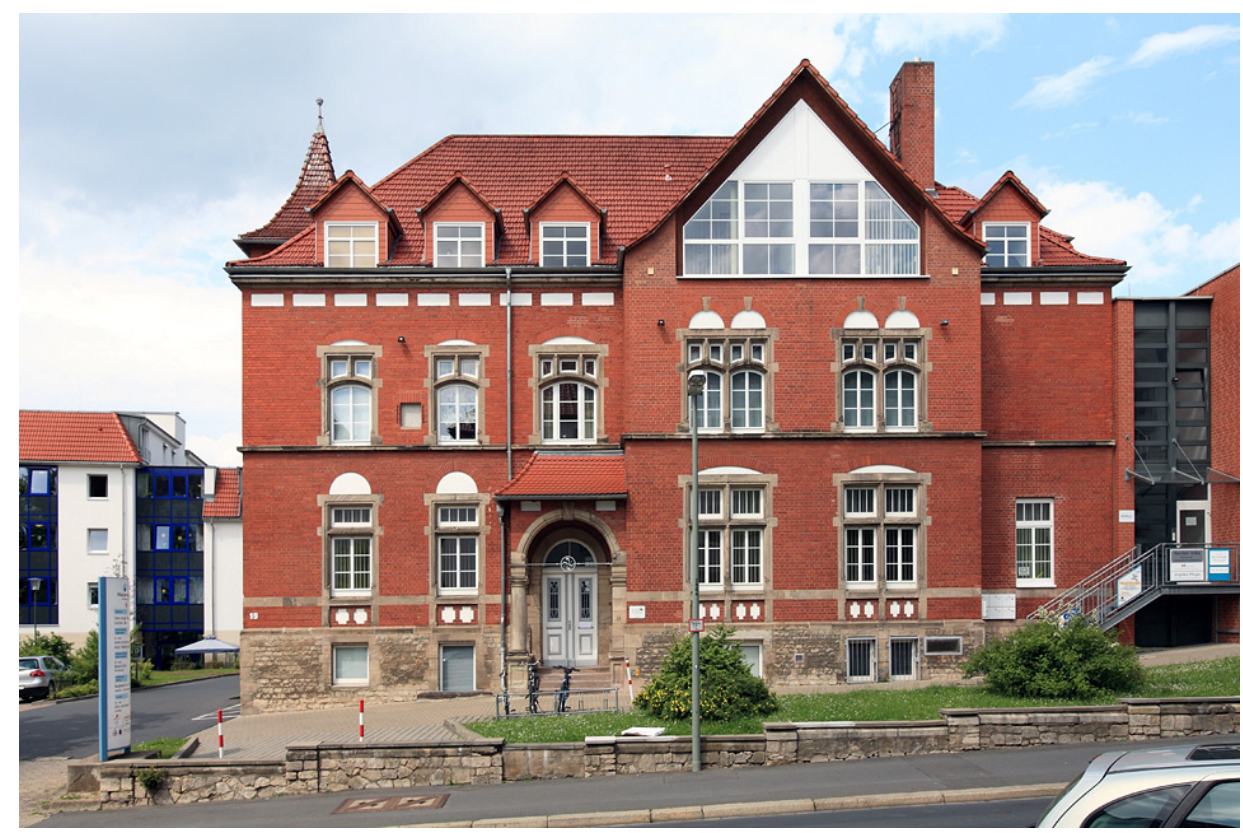

Abb. 27: Die Hautklinik (1928-1959)

Aufnahme 2009 (heute: Am Steinsgraben 19)

Ende März 1935 wurde Erhard Rieke emeritiert und Walther Krantz (1891-1970) sein Nachfolger. Ohne von der Fakultät vorgeschlagen zu sein, hatte ihn das Ministerium auf Betreiben des örtlichen NS-Dozentenbunds zum persönlichen Ordinarius und Direktor der Universitätsklinik für Haut- und Geschlechtskrankheiten ernannt. Am 1. April 1935 trat er sein Amt an. ${ }^{165}$ Steigende Patientenzahlen und die damit verbundene hohe Belegung führten sowohl in der Klinik am Steinsgraben als auch in der Poliklinik in der Absonderungsbaracke zu erheblichen Schwierigkeiten, die die bereits vorhandenen räumlichen und baulichen Mängel 
noch verschärften. Hinzu kam die räumliche Distanz zwischen den beiden Klinikbereichen, die den täglichen Ablauf und den zu erteilenden Unterricht stark beeinträchtigten. Ein Neubau, bei dem Klinik und Poliklinik unter einem Dach vereint sein sollten, wurde zu einer dringenden Forderung.

Mit Schreiben vom 13. Juni 1938 sagte dann das Ministerium einen Neubau zu und verwies darauf, dass im Haushalt 1939 ein Teilbetrag dafür veranschlagt werde. ${ }^{166}$ Der Plan für den Neubau sah eine Klinik mit 100 Betten, eine Poliklinik und einen Hörsaal vor. Der Beginn des Zweiten Weltkriegs verhinderte dann seine Durchführung.

Gleich zu Beginn des Zweiten Weltkriegs wurde mit Ausnahme der Frauenstation die Hautklinik durch die Wehrmacht in ein Reservelazarett umgewandelt. Im Frühjahr 1940 erfolgte dann wieder eine weitgehende Räumung, doch verblieb eine Station für hautkranke Soldaten zurück. Die Hautklinik selbst verfügte nun wieder über 50 Betten. Der Mangel an Personal führte ebenso wie die teilweise Unterbringung von Patienten in Göttinger Gasthäusern zu ganz erheblichen Missständen. Hinzu kam die Beschlagnahme des klinikeigenen PKWs, mit dem die Patienten zwischen Klinik und Poliklinik transportiert wurden, wodurch die durch die räumliche Trennung bedingten Schwierigkeiten verschärft wurden. Kurz vor Kriegsende, am 8. April 1945, schlugen drei Granaten ein; eine traf den Männerpavillon, doch konnten die sogleich eingeleiteten Löscharbeiten größeren Schaden verhindern.

Krantz wurde im November 1945 als politisch Belasteter unter Fortfall seiner Bezüge durch die britische Militärregierung aus dem Staatsdienst entfernt, im Oktober 1946 aber erneut in sein Amt eingesetzt, um umgehend ordnungsgemäß pensioniert werden zu können, einem damals nicht unüblichen Verfahren. ${ }^{167}$

Am 1. Februar 1946 übernahm Horst-Günther Bode (1904-1990) zunächst kommissarisch die Leitung der Hautklinik. Er hatte sich unter Riecke in Göttingen habilitiert, war aber inzwischen nach Breslau gegangen. Es gelang ihm, zunächst einige bauliche Verbesserungen durchzusetzen und vor allem die apparative Einrichtung zu verbessern. Glanzstück war ohne Zweifel die 1952 installierte Elektronenschleuder, die hier erstmals in Deutschland zur Therapie von Hautkarzinomen mit schnellen Elektronen eingesetzt wurde.

\section{Die Hautklinik am Kreuzbergring}

1959 war der Neubau der Hautklinik am Kreuzbergring vollendet und konnte im Mai bezogen werden. Auf einem Areal von $11000 \mathrm{~m}^{2}$ war in aufgelockerter Bauweise eine Klinik mit einem Betten- und Behandlungstrakt, zwei Polikliniken, einem Hörsaal und der Abteilung für die Elektronenschleuder entstanden. Ein wesentlicher Gedanke, der der Konzeption zugrunde lag, war die Trennung der Zugangswege für ambulante und stationäre Patienten. Die Funktionsräume hingegen konnten sowohl von den Stationen als auch von der Poliklinik aus benutzt werden. 
Das sechsstöckige Bettenhaus war mit insgesamt 122 Betten ausgestattet. Die Kinderstation im Erdgeschoss hatte 16 Betten, ein Spielzimmer und einen Tagesraum. Auf dieser Etage befand sich auch die Klinikapotheke. Je zwei Frauen- und Männerstationen mit jeweils 24 Betten befanden sich in den ersten vier Obergeschossen. Die Privatstation mit zehn Einzelzimmern war im fünften Obergeschoss untergebracht. Im Behandlungsgebäude befanden sich im Sockel- und Erdgeschoss die einschlägigen Laboratorien sowie Funktionsräume und im ersten Obergeschoss zwei Operationsräume, die Bibliothek und die beiden Dienstzimmer der Klinikleitung. Im Erdgeschoss der Poliklinik war die Frauen- und im Obergeschoss die Männerstation. Ferner gab es hier sechs Untersuchungs- und mehrere Funktionsräume. Der Hörsaal war im Inneren in der Form eines Amphitheaters gebaut mit steil übereinander geordneten Sitzreihen, die insgesamt 138 Sitzplätze boten. ${ }^{168}$

Das ursprünglich verwirklichte bautechnische Konzept besteht bis heute, wenn auch die strukturellen Positionen wie die Anzahl der Stationen oder Betten bis in die Gegenwart hinein Änderungen unterlagen. Auch führten wissenschaftliche Erkenntnisse zu neuen Therapieansätzen. So fiel die so erfolgreich gestartete Elektronenschleuder der operativen Therapie zum Opfer.

\section{Hals-Nasen-Ohren-Klinik}

Der Zusammenschluss dieser drei Teilfächer beruhte weniger auf den Gemeinsamkeiten in der Pathologie, Ätiologie oder Symptomatologie der zu behandelnden Krankheiten. „Gemeinsam war ihnen die Enge und Dunkelheit ibrer Arbeitsgebiete und damit deren Unzulänglichkeit für den Durchschnittschirurgen der Zeit um 1860, dazu das Hilfsmittel des reflektierten Lichts. Die zeitlich etwa parallel verlaufende Entwicklung von Otologie und Laryngologie fübrte schließlich zur Harmonie, nicht eigentlich zur Verschmelzung der im Grunde verscbiedenen Fächer. " 169

In Göttingen hatte bereits Himly 1809 über "Morbos organorum visus et auditus “ gelesen, doch als erster Fachvertreter galt Kurd Bürckner (1853-1913), der sich 1877 in Würzburg für das Fach Ohrenheilkunde habilitiert hatte und im Dezember des gleichen Jahres nach Göttingen berufen wurde.

Am 20. Februar 1878 wurde die ,königliche Universitäts-Poliklinik für Ohren- und Nasenkrankheiten zu Göttingen" eröffnet. Es war vor allem Bürckners Verdienst, dass er die bis dahin noch relativ bedeutungslose Rhinologie einbezog. Erst 1907 erhielt er auch einen Lehrauftrag für das Fach Laryngologie. Gleichzeitig wurde die bisherige Bezeichnung entsprechend erweitert und in Poliklinik für Hals-, Nasen- und Ohrenkranke umgewandelt.

Die Anfänge waren mehr als bescheiden. Der Poliklinik stand nur ein Zimmer im Erdgeschoss des Wrisbergschen Hauses zur Verfügung. Bürckner organisierte den Poliklinikbetrieb zunächst ohne Unterstützung von außen. Erst ab 1883 wur- 
de ihm ein jährlicher Staatszuschuss gewährt. Auch die Stadt Göttingen beteiligte sich mit einer jährlichen Beihilfe, allerdings unter der Bedingung, dass die armen Kranken Göttingens kostenlos behandelt wurden. Im Etatjahr 1884/85 wurde die Poliklinik dann in die staatliche Trägerschaft übernommen und Bürckner zum

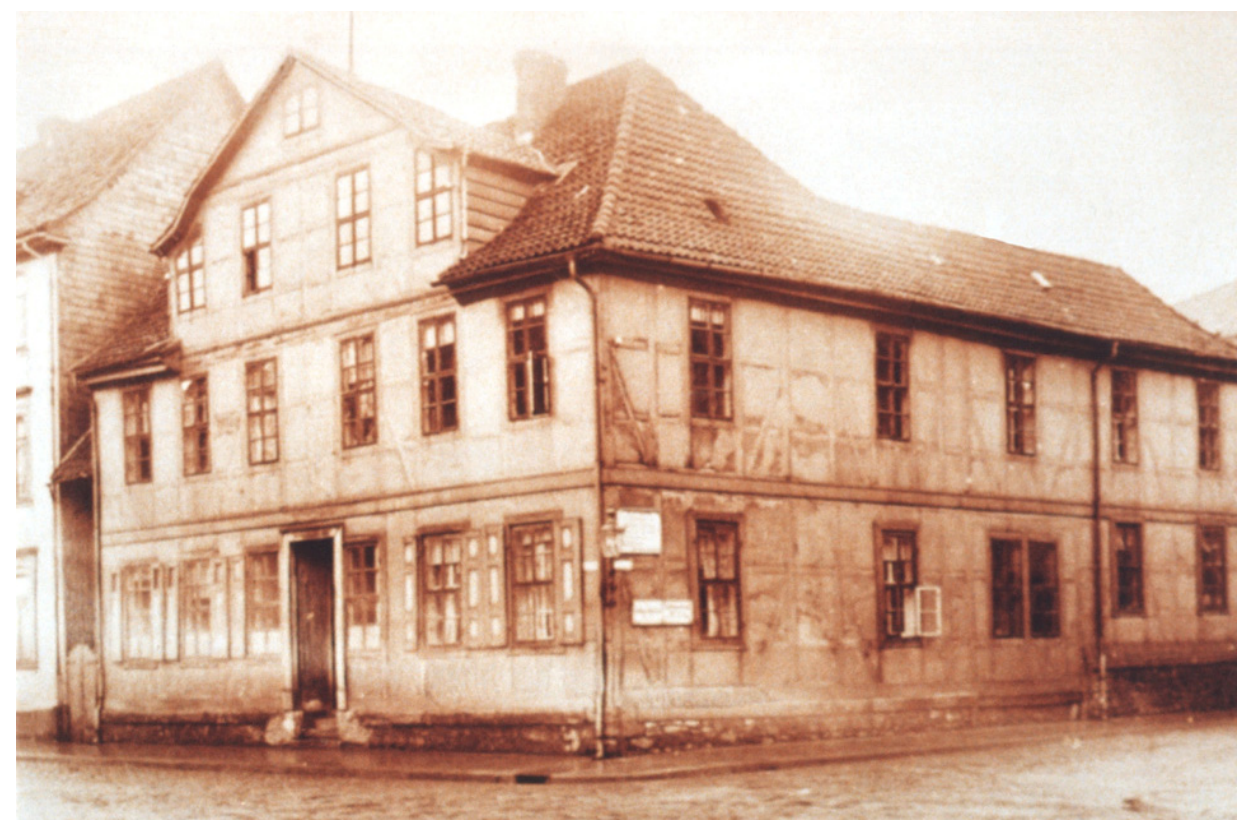

Abb. 28: Das ehemalige Wrisbergsche Haus

Ecke Geiststraße/Goetheallee

Anonyme Photographie, o. J.

Direktor und im Januar 1885 zum außerordentlichen Professor ernannt. ${ }^{170} \mathrm{Zu}-$ nächst hielt er seine Vorlesungen in der Privatwohnung; ab Sommersemester 1880 fanden sie dann im Behandlungszimmer der Klinik statt. Die Resonanz war allerdings eher mäßig. In wissenschaftlicher Hinsicht leistete Bürckner vor allem auf otologischem Gebiet Pionierarbeit. Besonders hervorzuheben sind in diesem Zusammenhang sein „Lebrbuch der Obrenheilkunde“ (1892) und sein „Atlas von Beleuchtungsbildern des Trommelfells", der 1890 in dritter verbesserter Auflage erschien und durch eine Anleitung zur Ohrenspiegel-Untersuchung erweitert war. ${ }^{171}$

Die beständig wachsende Inanspruchnahme der Poliklinik vor allem durch Nasenkranke erforderte eine Vergrößerung der Räumlichkeiten und so gelang am 25. Juni 1892 der Umzug in die ehemaligen Absonderungsbaracken des ErnstAugust-Hospitals, die nach geringen baulichen Veränderungen inzwischen gründ- 
lich renoviert worden waren. 1900 wurde erneut aus Kapazitätsgründen ein Anbau erstellt. 1906 schließlich erfolgte die Verlegung in den nördlichen Teil des Hauptgebäudes des Ernst-August-Hospitals, das die Augenklinik inzwischen verlassen hatte. Damit war die Grundlage für die hier dann 1929 eingerichtete Klinik geschaffen. ${ }^{172}$

Da die Poliklinik trotz mehrfacher Anträge auch weiterhin über keine eigenen Räumlichkeiten zur Behandlung klinisch Kranker verfügte, musste ein Teil von ihnen in die Chirurgie verlegt werden. Für die übrigen Patienten bestand aufgrund einer privaten Vereinbarung eine entsprechende Abteilung mit 40 Betten im katholischen Stift Maria-Hilf. ${ }^{173}$

Nach Bürckners überraschendem Tod Ende August 1913 wurde Wilhelm Lange (1875-1954) bereits zum Wintersemester 1913/14 berufen. Nicht zuletzt durch zeitbedingte Umstände waren trotz mehrerer Anträge vor allem im Hinblick auf die Errichtung einer stationären Abteilung keinerlei Verbesserungen in Sicht. Lange sah sich deshalb gezwungen, durch eine private Vereinbarung im evangelischen Stift Neu-Bethlehem eine kleine Bettenabteilung mit neun Krankenzimmern im Dachgeschoss für seine HNO-Patienten einzurichten. ${ }^{174}$ Lediglich seine Ernennung zum ordentlichen Professor 1919 stellte in gewisser Weise eine Aufwertung des Fachs dar. So war es nicht verwunderlich, dass Lange 1922 einen Ruf nach Bonn annahm, freilich nicht ohne Oskar Wagener (1878-1942) aus Marburg der Fakultät als seinen Nachfolger zu empfehlen. ${ }^{175}$

Wagener hatte in Bonn, Berlin und Kiel studiert, wo er 1902 promoviert wurde. 1909 habilitierte er sich in Berlin. Über Greifswald (1914) und Marburg (1917) kam er im Oktober 1922 nach Göttingen. In der Anfangsphase widmete sich Wagener verstärkt der Klinikgestaltung. Er setzte durch, dass die im Stift NeuBethlehem bereits vorhandenen Krankenzimmer renoviert und künftig von Seiten des Staates angemietet wurden. Gleichzeitig wurden im ersten Stock eine Bettenabteilung für die Privatpatienten des Klinikdirektors eingerichtet und für die tägliche Mitbenutzung des Operationssaales eine staatliche Beihilfe festgelegt. ${ }^{176}$ Ebenfalls 1923 wurde der Neubau der Poliklinik in Angriff genommen, verzögerte sich aber aufgrund der wirtschaftlichen Verhältnisse und wurde erst 1926 fertiggestellt. Die dadurch im Ernst-August-Hospital freigewordenen Räume konnten nun zur Klinik umgebaut werden. Sie wurde 1928 eröffnet und am 12. April 1929 offiziell übergeben. „Damit besaß Göttingen nun endlich als weitaus letəte der deutschen Universitäten eine HNO-Klinik." 177 Die Auslagerung ins Dachgeschoss des Stifts NeuBethlehem war mit Ausnahme der Privatstation damit beendet.

Gelungen war die gefundene Lösung allerdings nicht. Da die Poliklinik mit dem Hörsaal und die stationäre Abteilung auf verschiedenen Straßenseiten lagen, war die Vorstellung bettlägeriger Kranker unmöglich. Dieser Missstand konnte erst durch die Kooperation mit dem inzwischen direkt an die HNO-Klinik angrenzenden Pharmakologischen Institut im Jahre 1949 beseitigt werden. 1933 
wurden die Räume der Universitätsfechthalle zu einem Kurssaal umgebaut und der Poliklinik angegliedert.

1942 starb Wagener; Hermann Frenzel (1895-1967) wurde noch im gleichen Jahr sein Nachfolger. Frenzel hatte in Göttingen und Greifswald studiert und sich 1925 habilitiert. Seit 1935 war er bis zu seiner Berufung nach Göttingen 1942 Chefarzt der städtischen Hals-Nasen-Ohrenklinik in Dortmund. Am 28. August 1945 wurde er von amerikanischen Soldaten festgenommen und im Lager Westertimke bei Bremen interniert. Am 25. April 1946 kehrte Frenzel zurück, durfte ab dem 14. August wieder ärztlich tätig sein und leitete dann die Klinik bis Ende 1963, die am 1. Januar 1964 Adolf Miehlke (1917-2001) übernahm.

Wissenschaftlich hat Frenzel vor allem in der Vestibularisforschung Grundlegendes geleistet und die von ihm entwickelte Leuchtbrille zur Nystagmusforschung wird bis heute weltweit benutzt. ${ }^{178}$

In den folgenden Jahren wurde durch Umbau innerhalb des Klinikgebäudes der wissenschaftlichen Entwicklung Rechnung getragen. 1949 und 1955 konnten die Räume des inzwischen ausgezogenen Hygiene-Instituts und der Nervenklinik übernommen werden. Die bislang in Neu-Bethlehem ausgelagerte Privatstation wurde nun in das Klinikgebäude integriert und so eine zweckmäßige Gliederung erreicht. Zwischenzeitlich konnte die Bettenzahl bis auf 69 gesteigert werden. Der angestrebte Neubau einer eigenständigen HNO-Klinik im Bereich der anderen Kliniken blieb zwar weiterhin das Ziel, doch konnte die Planung trotz mehrerer Anläufe nicht realisiert werden. ${ }^{179}$ Erst mit dem Einzug in das Universitätsklinikum im November 1988 war das Ziel dann erreicht.

\section{Die Entwicklung der Psychiatrischen Klinik}

Als wissenschaftliches Fach hat sich die Psychiatrie wesentlich später etabliert, als es die alltäglichen Anforderungen einer praktischen Hilfestellung für die entsprechend leidenden Menschen eigentlich erforderten. Die Geisteskranken wurden innerhalb der Gesellschaft - wenn überhaupt - nur sehr zögerlich akzeptiert. Sie blieben weitgehend stigmatisiert und wurden unter Verlust ihrer Persönlichkeit in eine Außenseiterrolle gedrängt. In gewisser Weise übertrug sich eine solche Sichtweise auch auf diejenigen Ärzte, die sich diesen Kranken widmeten. Auch sie wurden mit Distanz betrachtet und ihre Klinik lag meist abseits, organisiert in eigener Verwaltung und Ökonomie.

Die wissenschaftliche Psychiatrie entstand nicht aus caritativen Überlegungen, sondern aus der Erkenntnis, dass geistig Leidende ebenso ärztlich zu behandeln seien wie körperlich Leidende. Die dafür nötige klinische Ausbildung in der Psychiatrie war allerdings ,nur und erst dann möglich, wenn entsprechende Anstalten in unmittelbarer Nähe der Universität verfügbar waren. " 180 
Seit 1866 war diese Voraussetzung in Göttingen gegeben. Die auf dem Leineberg in Rosdorf vor den Toren der Universitätsstadt errichtete ,provinzialständige Landes-Irrenanstalt" war die erste Anstalt in Deutschland, die ,mit der ausdrücklichen Vorbedingung des Unterrichts" errichtet worden war. ${ }^{181}$

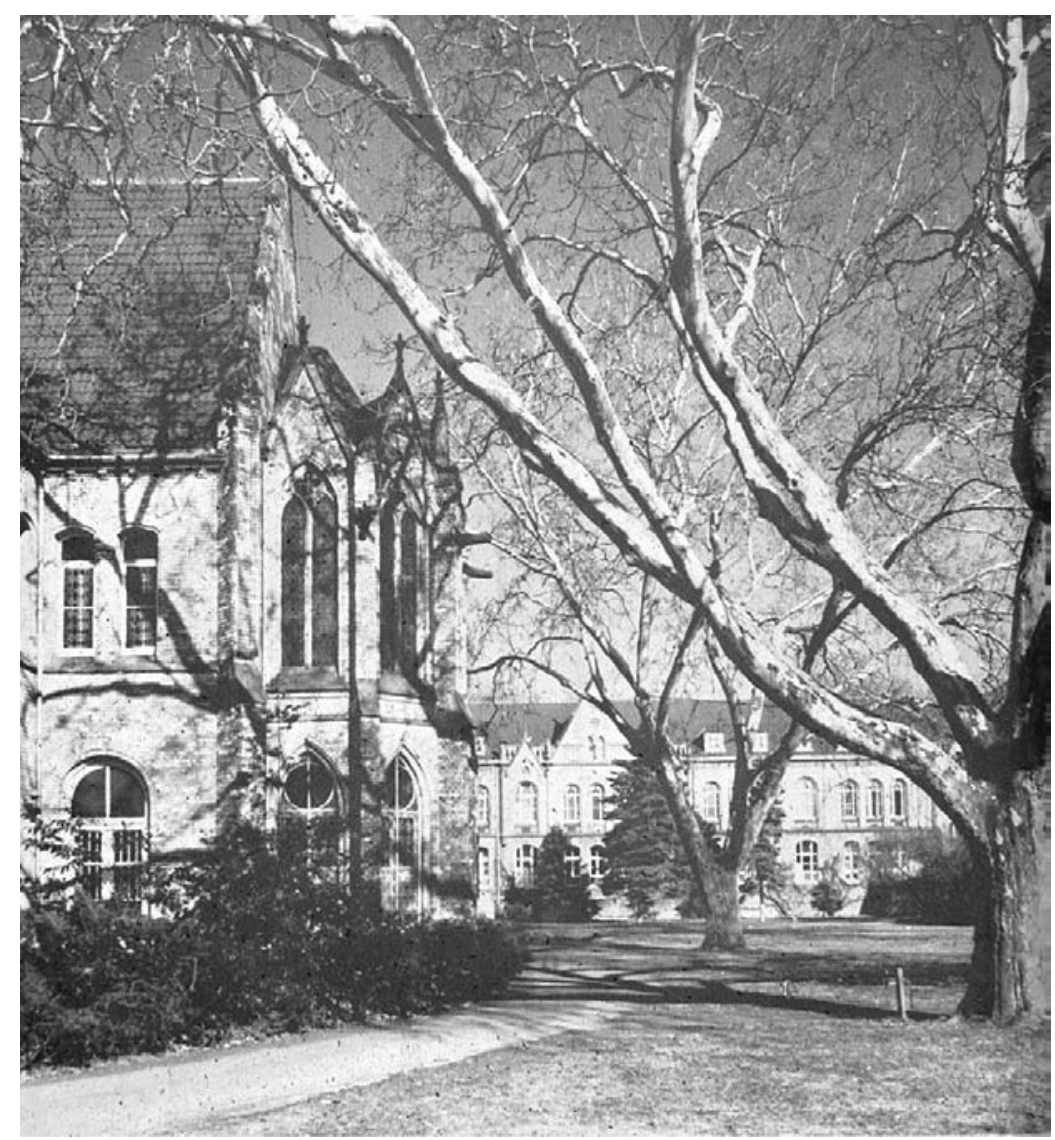

Abb. 29: Die ehemalige Provinzialständige

Landes-Irrenanstalt Rosdorf

Anonyme Photographie, o. J.

Am 5. April 1866 hatte der neu berufene Ordinarius für Psychiatrie Ludwig Meyer (1826-1900) in Personalunion ihre Leitung übernommen. Er hatte in Bonn, Würzburg und Berlin studiert, wo er 1852 promoviert wurde und sich 1858 habilitierte. Überzeugt von der Richtigkeit und Tragweite des No-Restraint-Verfahrens und erfüllt von der Idee einer humanen Irrenpflege wurde Meyer zum Pionier der modernen Psychiatrie. ${ }^{182}$ In Göttingen war er von Anfang an bestrebt, eine ,klini- 
sche Abteilung "aufzubauen, in der jeder Kranke bis zur Feststellung seines geistigen Zustandes unter ärztlicher Beobachtung bleiben sollte. Dieser Patientenkreis diente dann vorzugsweise dem klinischen Unterricht und stellte so die Verbindung der Anstalt mit der Universität her. ${ }^{183}$ Um die Nachteile abzumildern, die die räumliche Distanz zwischen Universität und Rosdorfer Anstalt für den klinischpsychiatrischen Unterricht mit sich brachte, wurde ein spezieller Pferdewagenverkehr für die Studenten eingerichtet, der bis nach dem ersten Weltkrieg bestand. Seine Finanzierung führte zu immer wiederkehrenden Diskussionen. ${ }^{184}$

Nach Meyers Tod wurde August Cramer (1860-1912) sein Nachfolger. Er war bereits seit 1. März 1895 als Oberarzt und stellvertretender Direktor in der Rosdorfer Anstalt. Bei seinem damaligen Amtsantritt hatte ihm die Medizinische Fakultät die Venia Legendi für Psychiatrie verliehen. Während seiner Amtsführung gelang es Cramer, das psychiatrisch-neurologische Arbeitsfeld in Göttingen bedeutend zu erweitern. 1901 eröffnete er eine Poliklinik für psychische und Nervenkrankheiten in gemieteten Räumen des sogenannten Wolfschen Hauses, das im Bereich Geist- und Alleestraße lag. Sie wurde zur Keimzelle der späteren psychiatrischen Universitätsklinik und Poliklinik. Am 1. Juli 1904 kam eine stationäre Abteilung mit zehn Betten hinzu und am 1. April 1906 wurden die Räume der alten Augenklinik bezogen. Fortan unterschied man in Göttingen zwischen der Anstalt in Rosdorf und der Klinik der Universität. Auf Cramers Initiative ging die Erweiterung der Anstalt um ein Provinzial-Verwahrungshaus für asoziale Geisteskranke, die Errichtung des Nervensanatoriums „Rasenmüble“ und die Gründung einer „Heil- und Erziehungsanstalt für psychopathische Fürsorgezöglinge“"zurück. ${ }^{185}$

Nach Cramers frühem Tod übernahm Ernst Schultze (1865-1938), der in Greifswald ordentlicher Professor für Psychiatrie war, die Leitung. Er hatte in Berlin und Bonn, wo er promoviert wurde, studiert. 1895 habilitierte er sich für Psychiatrie und Neurologie. Im Dezember 1912 trat er sein Amt in Göttingen an, gab aber die Leitung des Sanatoriums „Rasenmüble“ und der „Heil- und Erziehungsanstalt" ab, beschränkte sich folglich auf die Personalunion zwischen der Anstalt in Rosdorf und der Universität. Eine wichtige Aufgabe war der Ausbau einer stationären Abteilung. Der 1915 begonnene Pavillonneubau in der Geiststraße konnte erst 1919 in Betrieb genommen werden. 1928 wurden in der Anstalt am Leineberg ein spezieller Bereich für sachgemäße Sektionen und ein Laboratorium für feingewebliche Hirnuntersuchungen geschaffen. Durch seine Gutachtertätigkeit hat Schultze die Entwicklung der forensischen Psychiatrie entscheidend beeinflusst. Zu einer gewissen Berühmtheit gelangte er durch sein Gutachten im Fall des Massenmörders Fritz Haarmann. 1934 wurde Schultze entpflichtet, nachdem er bereits 1933 krankheitsbedingt das Direktorium der Rosdorfer Anstalt abgegeben hatte. ${ }^{186}$

Im Oktober 1934 übernahm Gottfried Ewald (1888-1963) wieder in Personalunion die Leitung der Provinzial-, Heil- und Pflegeanstalt und die Universitätsklinik für psychische und Nervenkranke mit dem entsprechenden Ordinariat. Auch 
er kam aus Greifswald. Ewald hatte in Erlangen und Heidelberg, wo er 1912 promoviert wurde, studiert. Während seines Sanitätsdienstes verlor er 1915 den linken Arm infolge einer septischen Infektion, die er sich bei der Leichensektion eines Meningitikers zugezogen hatte. Dies zwang ihn, sein zunächst angestrebtes Berufsziel „Innere Medizin“ zugunsten der Psychiatrie und Neurologie aufzugeben. 1920 habilitierte sich Ewald in Erlangen.

Zwei Tätigkeitsschwerpunkte bestimmten in der Anfangsphase seine Amtsführung. Zum einen versuchte er eine Trennung der ständig beschwerlicher werdenden und stark divergierenden Doppelaufgabe zwischen den beiden Kliniken zu erreichen. Trotz mehrerer diesbezüglicher Denkschriften gelang dies erst bei seiner Nachfolgeregelung 1956. Zum anderen war er entschieden darum bemüht, eine neue und zeitgemäße psychiatrische und neurologische Klinik zu schaffen. Die Bauplanungen und entsprechende Verhandlungen begannen 1935. Als Standort des Klinikgebäudes wurde der hintere Bereich des Kreuzbergweges festgelegt. Ende 1940 wurden ein erster erweiterter Entwurf vorgelegt und die ersten Geldraten etatisiert. Die Kriegsereignisse verhinderten jedoch eine zeitnahe Realisierung. Erst im Mai 1955 konnte der Neubau der Neurologischen und Psychiatrischen Klinik eröffnet werden, die als eine nach modernen Gesichtspunkten gestaltete Anlage begriffen wurde. ${ }^{187}$ Damit war nun auch die räumliche Trennung zur Rosdorfer Heil- und Pflegeanstalt vollzogen. 



\section{Zahnheilkunde}

Bis ins 20. Jahrhundert hinein führte die Zahnheilkunde innerhalb und außerhalb der Medizinischen Fakultät ein weitgehendes Eigenleben in der Rolle eines AuBenseiters. Bis 1909 wurde ein akademischer Bildungsstand nicht für nötig erachtet. An der Entstehung eines akademischen Zahnärztestandes hatte die Medizinische Fakultät zunächst keinen wesentlichen Anteil, die Initiative dazu ging vielmehr von den Zahnärzten selbst aus, die Anschluss an die Medizinische Fakultät suchten ${ }^{188}$ und 1919 die volle akademische Integration erreichten. ${ }^{189}$ An der Universität Göttingen begann die Zahnheilkunde mit dem Sommersemester 1821. Das Vorlesungsverzeichnis enthielt erstmals die Ankündigung: „Die Zabnkrankheiten, nebst einer practischen Anleitung zu den dabey vorkommenden Operationen, handelt $\mathrm{Hr}$. D. Pauli in einer noch zu bestimmenden Stunde ab. "190 Johann Heinrich Pauli (17951850) hatte wahrscheinlich seit 1811 in Göttingen studiert. Zu diesem Zeitpunkt war er als Gehilfe am Hospital und am anatomischen Theater nachgewiesen, in der Matrikel hingegen erst seit dem Wintersemester 1814/15. 1815 wurde er aufgrund von Gutachten seiner Göttinger Lehrer in Jena promoviert und im Sommersemester 1818 kündigte Pauli erstmals als Privatdozent an. ${ }^{191}$ Formell war er Privatdozent der Chirurgie und Mitarbeiter Langenbecks. Die Zahnheilkunde war also noch Bestandteil der Chirurgie.

In den folgenden Jahren kündigte Pauli sowohl im Sommer- als auch im Wintersemester seine zahnärztliche Vorlesung in identischer Formulierung an. Erst zum Sommersemester 1832 erfolgte die Einbeziehung der Zahnersatzkunde: „Eine Anleitung zu der Bebandlung der Zabnkrankheiten und den dabey erforderlichen Operationen, sowie auch zu der Verfertigung und Einsetzung künstlicher Zähne oder ganzer Gebisse gibt derselbe privatissime. " 192

Ein Semester später fügte er bei den Gebissen „aus Email“ hinzu, womit wohl Porzellanzähne gemeint waren. In dieser Form erfolgte dann die Ankündigung bis zu Paulis Tod am 30. Januar 1850.

Neben seiner Tätigkeit als Privatdozent unterhielt Pauli eine umfangreiche und wohl auch einträgliche zahnärztliche Privatpraxis ${ }^{193}$ und spiegelte so ein Arztbild wider, das für Göttingen in jener Zeit signifikant war. „Besondere Zabnäržte zur Bebandlung der Zabnkrankheiten sind bier nicht, einige Wundärzte jedoch baben in diesem Zweig der Heilkunde Geschicklichkeit und Fertigkeit erlangt. "194 
Nach Paulis Tod ruhte der zahnärztliche Unterricht zunächst. Erst 1894 wurde der praktische Zahnarzt Karl Heitmüller (1864-1951) durch den Erlass vom 15. Januar zum Lehrer der Zahnheilkunde an der Universität ernannt. ${ }^{195}$

Heitmüller hatte in Berlin ein zweisemestriges zahnärztliches Studium absolviert und 1887 mit dem Staatsexamen abgeschlossen. 1890 erwarb er in den USA den Titel „Doctor of Dental Surgery (D. D. S.)“. Bereits 1887 hatte er in Göttingen eine zahnärztliche Praxis eröffnet, die regen Zuspruch erhielt und ihm auf Grund seiner Geschicklichkeit hohes Ansehen verlieh. ${ }^{196}$

Der Unterricht sollte sich zunächst eng an die chirurgische Poliklinik anlehnen und als „eine Art Nebenzweig“ der Chirurgie betrieben werden. Die klinischen Demonstrationen über die Krankheiten der Mundhöhle wurden von einem Arzt abgehalten, die speziell zahnärztlichen Unterweisungen erteilte der Zahnarzt. Da in der Chirurgie kein Platz vorhanden war, musste Heitmüller für die Räumlichkeiten selbst sorgen. ${ }^{197}$ Insgesamt stand die Fakultät seinem Wirken eher zurückhaltend gegenüber. Erst 1908 wurde ihm der Professorentitel verliehen, der allerdings keine akademischen Ansprüche begründete. ${ }^{198}$

Bereits 1893 hatte das Ministerium die Einrichtung eines staatlichen zahnärztlichen Instituts abgelehnt und jegliche finanzielle Zuwendung ausgeschlossen. ${ }^{199}$ „Die Poliklinik für Zahnkranke “des Lehrers der Zahnheilkunde Dr. chir. dent. Heitmüller am Theaterplatz war eine rein private Einrichtung, die von seinem Leiter aus eigenen Mitteln aufgebaut und finanziert wurde. Er hatte sein Wohnhaus, in dem sich die Privatpraxis befand, durch einen einstöckigen Anbau erweitert. Darin wurden ein Behandlungsraum mit vier Operationsstühlen und ein kleines Wartezimmer eingerichtet. Die „technische Abteilung“, die aus zwei zahntechnischen Laboratorien bestand, war im daneben liegenden Wohnhaus untergebracht. ${ }^{200}$

Der zahnärztliche Lehrbetrieb begann im Sommersemester 1894. Angeboten wurden eine zweistündige Vorlesung über „Pathologie und Therapie der Zahn- und Mundkrankheiten“, ein Praktikum von neun Wochenstunden ,in der Behandlung erkrankter Zähne und Conservierung derselben durch die Füllung" sowie ein ,zahntechnisches Praktikum "von 42 Wochenstunden. Julius Rosenbach (1842-1923) aus der chirurgischen Poliklinik las in zwei Wochenstunden ,chirurgische Poliklinik für Studierende der Zahnheilkunde mit besonderer Berücksichtigung der Krankheiten der Mundhöhle". Drei Studenten besuchten die Heitmüllerschen Vorlesungen und Praktika. 494 Patienten wurden dabei behandelt. ${ }^{201}$

Die 1898 aufgrund gestiegener Teilnehmerzahl vorgenommenen Erweiterungen musste Heitmüller wiederum ohne staatliche Unterstützung durchführen. Der Anbau wurde um ein weiteres Stockwerk erhöht. Im Erdgeschoss waren nun die zahntechnische Abteilung mit einem Raum für prothetische Behandlung sowie ein Labor mit sechs Arbeitsplätzen für Studenten und einem für den Assistenten eingerichtet. Im Obergeschoss befanden sich ein kleines Zimmer für die klinischen 
Untersuchungen der Patienten und die Abteilung für Zahnfüllungen. Ferner wurde das nun benötigte Inventar angeschafft.

Als Heitmüller am 15. November 1899 die Mitglieder der Medizinischen Fakultät zur Besichtigung seiner erweiterten und neu eingerichteten zahnärztlichen Poliklinik einlud, folgten nur zwei ungenannt gebliebene Fakultätsmitglieder der Einladung. ${ }^{202}$ Ein solch brüskierendes Verhalten offenbarte in aller Deutlichkeit, welch ablehnende Haltung die Medizinische Fakultät dem Lehrer der Zahnheilkunde entgegenbrachte. Auch in den folgenden Jahren ließ das Bestreben der Medizinischen Fakultät nicht nach, Heitmüller loszuwerden. Zu seinen schärfsten Gegnern innerhalb der Fakultät zählten Max Runge und Wilhelm Ebstein. Ihre Kritik basierte in erster Linie auf den als überzogen betrachteten Geldforderungen Heitmüllers.

Nach drei vergeblichen Gesuchen wurden Heitmüllers sichtbare Bemühungen 1900 dennoch von Erfolg gekrönt, denn unter Umgehung der Fakultät erreichte er in direkten Verhandlungen in Berlin neben der Anerkennung als Universitätsinstitut auch eine staatliche Subvention von jährlich 1000 Mark, die bereits im folgenden Jahr auf 1300 Mark erhöht wurde. ${ }^{203}$

Durch einen erneuten Umbau konnten 1904 die zahntechnische Abteilung im Erdgeschoss auf nun 15 Arbeitsplätze erweitert und der Warteraum im Obergeschoss vergrößert werden. Ferner diente die Baumaßnahme dazu, die geäußerten Bedenken hinsichtlich der Anti- und Asepsis zu zerstreuen. ${ }^{204} 1908$ richtete Heitmüller im zweiten Stock seines Wohnhauses auch einen eigenen Hörsaal ein. ${ }^{205}$

Am 16. Juni 1910 jedoch teilte Heitmüller dem Ministerium resignierend mit, dass er sich der Aufgabe nicht mehr gewachsen fühle und zum Herbst seine Tätigkeit aufgeben werde. Zu diesem Schritt hatten sicherlich mehrere Faktoren beigetragen. Zum einen erwuchs aus der neuen Prüfungsordnung, die am 1. Oktober 1909 in Kraft getreten war, ein deutlicher Anstieg der Lehraufgaben, und das Ministerium war nicht bereit, eine zweite Assistentenstelle, die Entlastung gebracht hätte, zu finanzieren. Zum anderen hatte die Festsetzung der Studiendauer auf sieben Semester eine Abnahme der Studentenzahl zur Folge, die zu finanziellen Einbußen bei den Kollegiengeldern führte. Im Alter von 46 Jahren baute sich Heitmüller dann in Dresden eine neue Praxis auf. ${ }^{206}$

Der Zahnarzt Georg Riecke (1888-1968), ein ehemaliger Assistent Heitmüllers, übernahm zunächst die Leitung des Instituts in den nun angemieteten bisherigen Räumen. Mehrere Versuche der Medizinischen Fakultät, einen geeigneten Nachfolger zu berufen, scheiterten, da die Ministerialbürokratie kein Interesse an einer Fortführung des zahnärztlichen Unterrichts in Göttingen hegte, infolgedessen einen neuen Lehrauftrag verweigerte und die finanzielle Unterstützung strich. Am 1. Oktober 1911 wurde die von Heitmüller aufgebaute „Poliklinik für Zahnkranke" geschlossen und in den folgenden Jahren war der zahnärztliche Unterricht an der Universität Göttingen nicht mehr möglich. ${ }^{207}$ 
Unmittelbar nach dem Ende des Ersten Weltkrieges signalisierte das Ministerium am 12. November 1918 die Bereitschaft, in Göttingen ein zahnärztliches Institut neu zu gründen. Auslöser war ein durch den Umzug des mathematischen Instituts freigewordenes Haus in der Bürgerstraße. Zwar war die Raumfrage geklärt, doch die Besetzung der Stelle durch ein entsprechendes Berufungsverfahren zog sich hin, sodass zunächst nur eine interimistische Lösung gefunden wurde. Am 14. November 1919 konnte das zahnärztliche Institut eröffnet werden. Mit seiner Leitung und der Durchführung des Unterrichts wurde Wilhelm Kunzendorf (1882-1948) beauftragt. Er hatte nach einem sechssemestrigem Studium 1908 das Staatsexamen abgelegt und war danach Assistent am zahnärztlichen Institut in Berlin, wohin er nach Beendigung seines Lehrauftrages Ende September 1920 zurückkehrte. 208
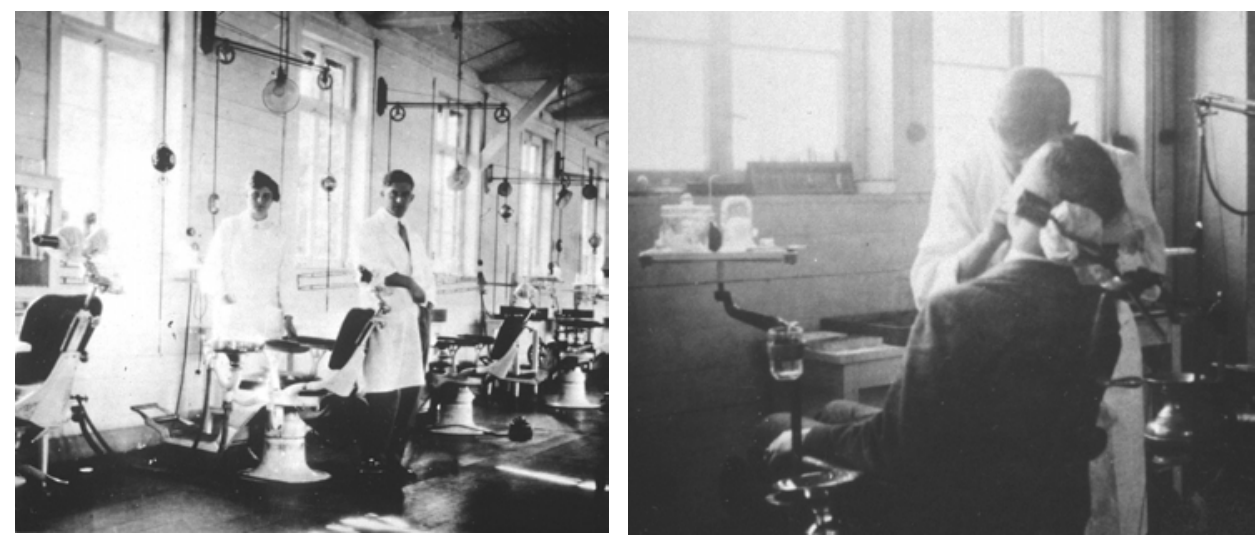

Abb. 30: „Füllabteilung“in der Institutsbaracke zu Zeiten Rebels

Am 6. Oktober wurde Hermann Euler (1878-1961) berufen. Er wurde am 29. März 1921 zum außerordentlichen Professor an der Medizinischen Fakultät ernannt und trat sein Amt zu Beginn des Sommersemesters an. ${ }^{209}$ Euler hatte in Erlangen, Heidelberg sowie Freiburg studiert und wurde 1902 in Erlangen zum Dr. med. promoviert. 1904 begann er das Studium der Zahnheilkunde am Heidelberger Institut, legte 1905 das Staatsexamen ab und habilitierte sich 1907. Im Herbst 1910 folgte die Berufung als Extraordinarius nach Erlangen. 1919 verlieh ihm die Medizinische Fakultät den Dr. med. dent. h. c.. Schwerpunkte seines umfangreichen wissenschaftlichen Werkes lagen auf zahnärztlich-pathohistologischen und zahnärztlich-chirurgischen Themen. ${ }^{210}$

Göttingen war für Euler nur eine Etappe, denn bereits im Wintersemester 1924 nahm er einen Ruf nach Breslau an. Nachfolger wurde sein Assistent HansHermann Rebel (1889-1967), der am 2. Februar 1925 zum persönlichen Ordinarius und Direktor des zahnärztlichen Instituts ernannt wurde. ${ }^{211}$ 
Rebel hatte in München Zahnmedizin studiert, erhielt 1914 die Approbation und war Assistent an der konservierenden Abteilung des Münchener Instituts. Im Dezember 1920 wurde er in Erlangen bei Euler promoviert und erwarb dadurch als einer der ersten den Titel „Dr. med. dent.“. 1922, inzwischen Assistent in Göttingen, habilitierte er sich an der Medizinischen Fakultät. ${ }^{212}$ Unter der NS-Diktatur war Rebel Pressionen ausgesetzt. Durch Einsprüche der Gauleitung und des örtlichen NS-Dozentenbundes wurden ihm Besuche ausländischer Kongresse untersagt sowie die Annahme der an ihn ergangenen Rufe nach Marburg (1933) und Freiburg (1934) verhindert. Seit Beginn des Zweiten Weltkrieges war die Kieferverletztenabteilung des Göttinger Reservelazaretts dem zahnärztlichen Institut unter der Leitung Rebels eingegliedert. ${ }^{213} 1947$ folgte Rebel dem Ruf nach Tübingen, wo er zum Ordinarius ernannt wurde. ${ }^{214}$

Im April 1948 übernahm Wilhelm Meyer (1896-1982) zunächst kommissarisch die Leitung des Instituts. Im August wurde er dann zum ersten planmäßigen Ordinarius an der Medizinischen Fakultät und zum Direktor des zahnärztlichen Instituts ernannt. Meyer hatte in Marburg und Göttingen Zahnmedizin studiert. 1921 legte er das Staatsexamen ab und wurde im gleichen Jahr zum Dr. med. dent. promoviert. 1925 habilitierte er sich in Breslau und wurde 1930 zum außerordentlichen Professor ernannt. ${ }^{215}$ Während Meyers Amtszeit wurde nicht nur die Personalstruktur deutlich verbessert, sondern auch die räumliche Situation. So wurde die Zahl der Assistenten von einem auf drei erhöht und Anfang Oktober 1957 konnte die „Zahnärztliche Klinik und Poliklinik", wie das Institut nun offiziell hieß, in der alten Nervenklinik in der Geiststraße eröffnet werden. Der vorklinische Teil der Ausbildung und der Phantomkurs der Zahnerhaltung blieben allerdings weiterhin in der Bürgerstraße. Für die klinische Ausbildung waren im Kellergeschoss ein Laboratorium mit 40 Arbeitsplätzen eingerichtet. Der Hörsaal mit 100 Sitzplätzen lag im Obergeschoss. Durch die nun in größerer Zahl vorhandenen Räume konnten eine vom prothetischen Bereich getrennte eigenständige kieferorthopädische Abteilung und eine Krankenstation mit 13 Betten eingerichtet werden. ${ }^{216}$ Das ehemalige zahnärztliche Institut war nun eine wirkliche Klinik geworden.

1976 wurde Wilhelm Meyer emeritiert und mit der Albrecht von HallerMedaille, der höchsten Auszeichnung, die die Medizinische Fakultät Göttingen verleiht, geehrt. ${ }^{217}$

Sein Nachfolger wurde Theodor Kirsch (1912-1997), der im Oktober 1968 den Ruf angenommen hatte. Kirsch hatte in Heidelberg und Marburg Medizin und Zahnmedizin studiert und sich in Heidelberg 1953 habilitiert. 1958 wurde er zum außerplanmäßigen Professor ernannt und 1964 auf das Extraordinariat für Kieferchirurgie nach Mainz berufen. Während seiner Amtszeit wurde die offizielle Bezeichnung in „Klinik und Poliklinik für Zahn-, Mund- und Kieferkrankheiten“ geändert und 1977 der Umzug in das neue Universitätsklinikum vollzogen. Am 31. März 1980 wurde Kirsch emeritiert. ${ }^{218}$ 



\section{Die Pathologie und ihre Institutionalisierung}

Am 1. April 1891, also noch vor der Frauen- und Augenklinik, wurde auf dem Gebiet der neu erbauten Kliniken das Pathologische Institut eröffnet. Damit stand auch für dieses Fach ein angemessenes und funktionstüchtiges Gebäude zur Verfügung. Der noch etwas provisorische Charakter des ersten 1850 auf dem Hinterhof des Ernst-August-Spitals errichteten Instituts war damit überwunden.

Die Pathologie war, bevor sie sich als eigenständiges Spezialfach etablierte, in ihrer Anfangsphase eng mit der Anatomie verknüpft. Um die Ursache einer tödlich verlaufenden Krankheit herauszufinden, war eine entsprechende Leichenöffnung unumgänglich. Während sich das medizinische Denken ursprünglich damit begnügte, pathologisch-anatomische Befunde lediglich festzustellen, begann es unter dem Einfluss der Naturwissenschaften zu Beginn des 19. Jahrhunderts die pathologischen Veränderungen einzelner anatomischer Befunde zueinander in Beziehung zu setzen und induktiv Gesetzmäßigkeiten zu erkennen, nach welchen sich pathologische Veränderungen vollziehen. ${ }^{219}$ An der Medizinischen Fakultät in Göttingen hatte Heinrich August Wrisberg für das Sommersemester 1788 die erste pathologisch-anatomische Vorlesung angekündigt. Sie war zweistündig pro Woche angesetzt, dürfte aber, da jegliche spezifischen Voraussetzungen gefehlt haben, rein theoretisch konzipiert gewesen sein. Durchgehend bis zu seinem Tode 1808 hatte Wrisberg dann diese Vorlesung gehalten. ${ }^{220}$

In den folgenden Jahren setzten mehrere Privatdozenten die Vorlesung fort; allerdings ohne Regelmäßigkeit und vielmehr ,nur nebenbei und in rein theoretischer Weise. "221 Erst zu Beginn der vierziger Jahre des 19. Jahrhunderts erhielt das Spezialfach einen festen Platz im Lehrplan, wozu zwei Ereignisse entscheidend beitrugen. Am 1. September 1840 stimmte das Kuratorium den Vorschlägen von Conrad Heinrich Fuchs zu, wonach den Angehörigen der in der Anstalt Verstorbenen das Recht abgesprochen wurde, die Leichenöffnung zu verweigern. Dieser Entschluss sollte den Angehörigen bereits vor der Aufnahme mitgeteilt werden. Das zweite in dieser Entwicklung positive Ereignis war die Habilitation Bernhard Langenbecks (1810-1887) im Mai 1838 für Physiologie und pathologische Anatomie. 1841 wurde er zum außerordentlichen Professor ernannt mit der Verpflichtung, über pathologische Anatomie und die Verrichtung legaler Leichenöffnungen zu lesen. Somit galt Langenbeck als erster Lehrer einer etablierten Pathologie in Göttingen. ${ }^{222} 1876$ wurde dann Emil Ponfick (1844-1913) erster Ordinarius in 
diesem Spezialfach. Unter seinem Nachfolger Johannes Orth (1847-1923), der 1878 sein Amt antrat, wurde der Neubau des Pathologischen Instituts durchgeführt, ein auch nach außen sichtbares Zeichen der inzwischen erreichten Institutionalisierung dieses Spezialfaches.

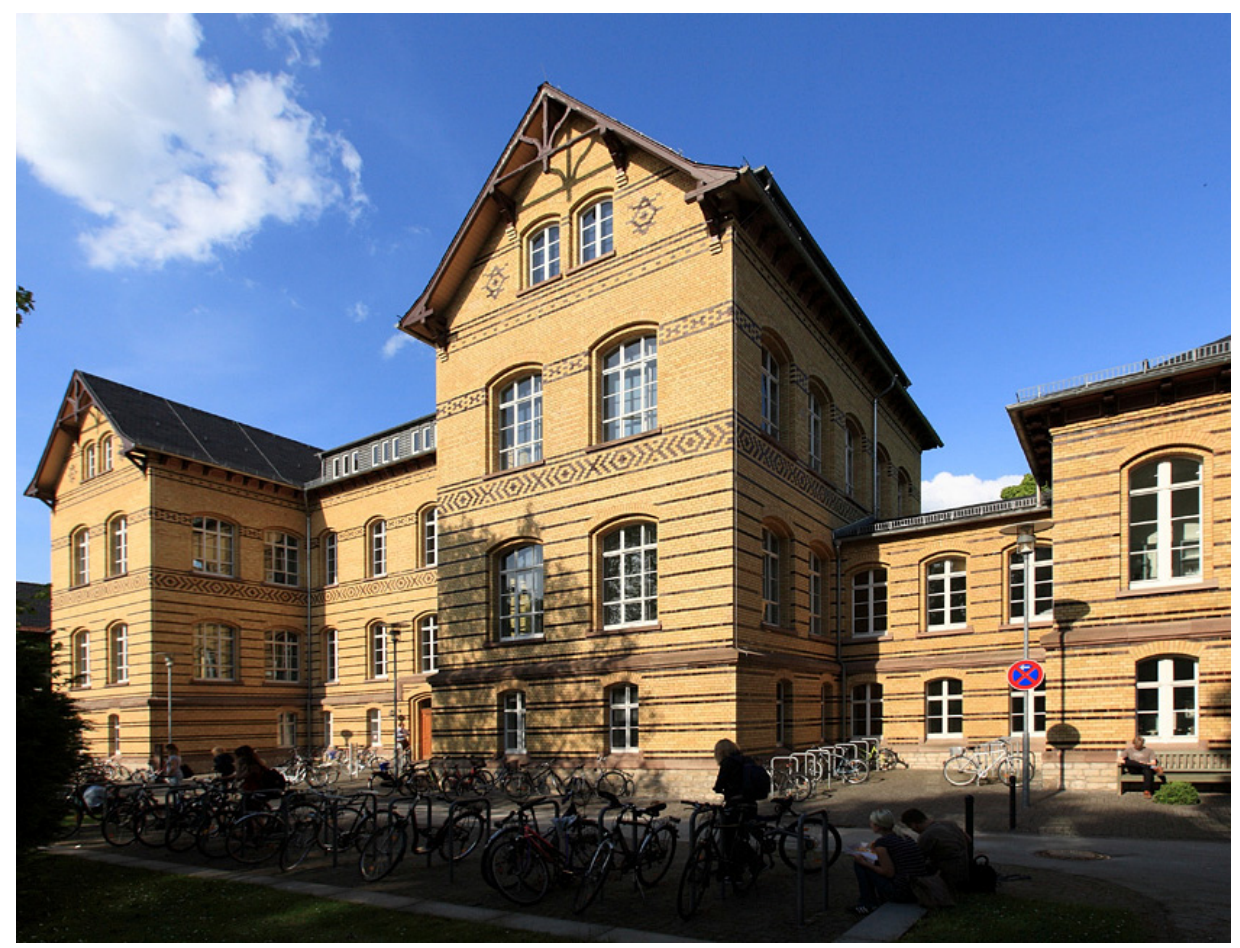

Abb. 31: Das Pathologische Institut (1891-1978)

(Westansicht des Hauptgebäudes)

Aufnahme 2009 (heute: Goßlerstraße 10)

Das Gebäude bestand aus zwei durch Gänge miteinander verbundenen Teilen. Das eigentliche pathologische Institut war im dreigeschossigen Hauptgebäude untergebracht. Im zweigeschossigen Nebengebäude befanden sich die zur Aufbewahrung, Untersuchung und Beerdigung der Leichen und Leichenteile nötigen Räume. Diese Zweiteilung brachte erhebliche Vorteile. Zum einen war der gesamte Bereich der Verstorbenen und der damit zusammenhängende Besucherverkehr vom Institut mit seinen Unterrichts- und Arbeitsräumen abgetrennt. Zum anderen konnte das Hauptgebäude von üblen Gerüchen und Mikroorganismen möglichst freigehalten werden. 
Das Hauptgebäude mit den beiden Seitenflügeln besaß im Erdgeschoss eine Dienerwohnung, einen Raum für einen großen Vegetationskasten und Tierställe. Im ersten Stock befanden sich ein großer Demonstrations- und Mikroskopiersaal sowie mehrere einschlägige Funktionszimmer. In der zweiten Etage waren der Hörsaal für 70 Zuhörer, das Dienstzimmer des Direktors und die Institutsbibliothek. Zwei völlig abgetrennte Räume dienten bakteriologischen Arbeiten und der gesamte übrige Bereich war der Sammlung der Weichteilpräparate vorbehalten. Die Knochensammlung wurde im Dachgeschoss aufbewahrt.

Das zweigeschossige Nebengebäude besaß im Erdgeschoss den Leichenkeller, der mit einer Berieselungsanlage für die Kadaver und zwei gemauerten Eissärgen ausgestattet war, eine würdige Begräbniskapelle und mehrere Funktionsräume, darunter einen kleineren Keller für Leichen, deren Todesursache gerichtlich festgestellt werden musste. Im ersten Stock befanden sich neben mehreren Funktionsräumen der für die chirurgischen Operationskurse bestimmte Saal und ein Seziersaal mit amphitheaterartig angeordneten Stehplätzen für ungefähr $100 \mathrm{Be}-$ trachter, die über eine besondere Seitentreppe Zugang fanden. Auf dem Dach war eine Knochenbleiche eingerichtet. ${ }^{223}$ Bis zum Einzug ins Universitätsklinikum 1978 verblieb das Pathologische Institut in diesem Gebäudekomplex. 



\section{Jüngere Spezialfächer}

Die Medizin in ihrer Vielschichtigkeit zeigt unter historischem Blickwinkel betrachtet einen beständigen Fluss hin zur konsequenten Spezialisierung. Die unter dem Einfluss der Naturwissenschaften im 19. Jahrhundert gewonnenen wissenschaftlichen Erkenntnisse und deren Problematisierung ließen einschlägige Spezialfächer entstehen, die durch ihre Institutionalisierung eine entsprechende Aufwertung erfuhren. Wie sich aus der Physiologie Spezialfächer entwickelten und sich dieser Prozess konkret an der Medizinischen Fakultät in Göttingen vollzog, soll exemplarisch am Beispiel der Physiologischen Chemie und der Hygiene gezeigt werden. Die Pharmakologie hingegen dokumentiert signifikant die Einbindung eines eigenständigen und auf langer Tradition beruhenden Faches in die Medizinische Fakultät.

\section{Physiologische Chemie und Hygiene}

Dass die Chemie einer Medizinischen Fakultät zur Blüte gereicht, hatte schon Werlhoff 1733 in seinem Gutachten zur Errichtung der Göttinger Fakultät festgehalten. Das Interesse der Ärzte an chemischen Fragestellungen im Zusammenhang mit der Heilkunde ist seit Paracelsus (1493/94-1541), den Paracelsisten und der Iatrochemie durchgängig dokumentiert. Obwohl die chemische Forschung in der Folgezeit den unmittelbaren medizinischen Aufgabenbereich allmählich überschritt und sich zunehmend zu einem eigenständigen Fach entwickelte, blieb sie bis weit ins 19. Jahrhundert hinein eng mit der Medizin verknüpft, wie das Beispiel des bedeutenden Chemikers Friedrich Wöhler zeigt, der bekanntlich bis zu seinem Tod der Medizinischen Fakultät in Göttingen angehörte. 224

Um die Mitte des 19. Jahrhunderts entstanden unter dem Einfluss neu entwickelter chemischer Methoden in medizinischen Fakultäten chemische Laboratorien, die die Keimzellen des späteren medizinischen Spezialfaches wurden. Die volle und endgültige Emanzipation der physiologischen Chemie erfolgte im 20. Jahrhundert. ${ }^{225}$ Das neue Fach „Biochemie“ erfuhr dann in der Folgezeit bedingt durch die wissenschaftliche Entwicklung weitere Differenzierungen.

In Göttingen wurde 1881 im Physiologischen Institut, das zu jener Zeit unter der Leitung von Georg Meissner stand, für Carl Flügge (1847-1923) eine besondere Abteilung für Physiologische Chemie eingerichtet. Ab dem Wintersemester 1881/82 
kündigte er eine einschlägige Vorlesung an. Bis zu diesem Zeitpunkt leitete der Extraordinarius der Philosophischen Fakultät Karl Boedeker (1815-1895) die „praktisch-chemischen Uebungen im physiologisch-chemischen Laboratorium. " 226

Flügge hatte in Göttingen zunächst Medizin studiert und wurde 1869 promoviert. 1874 begann er das Studium der Chemie in Bonn, setzte es in Göttingen fort und übernahm 1877 die Leitung des Instituts für Physiologische Chemie in Leipzig. 1878 habilitierte er sich in Berlin und 1881 erfolgte die Umhabilitation nach Göttingen. 1882 wurde er zum Extraordinarius und 1885 zum Ordinarius für „Medizinische Chemie und Hygiene“ ernannt. ${ }^{227}$ Die entsprechende Bezeichnung trug auch sein Institut, das im April 1883 in den von der Medizinischen Fakultät angemieteten Räumen des Walkenrieder Schäferhofes in der Kurzen Geismarstraße eingerichtet wurde. Das ehemalige Wohnhaus besaß einen Keller, zwei Geschosse und einen Dachboden. Während der Keller überwiegend als Vorratslager diente, waren im Erdgeschoss sechs Funktionsräume. Im Obergeschoss befanden sich der Hörsaal, in dem auch der bakteriologische Unterricht stattfand, und fünf Arbeitszimmer.

Die räumliche Enge und die unzureichende Ausstattung erschwerten das Arbeiten ganz erheblich und erweckten insgesamt den Eindruck des Provisorischen. Doch sollte es noch acht Jahre dauern, bis eine Besserung eintrat. Inzwischen hatte Flügge 1887 einen Ruf nach Breslau angenommen und Göttingen verlassen. ${ }^{228}$ Seinen Nachfolger, Gustav Wolfhügel (1845-1899), hatte Flügge selbst der Fakultät vorgeschlagen. Dieser hatte Chemie und Medizin in Heidelberg und Würzburg studiert, wo er 1869 promoviert wurde. 1876 habilitierte er sich in München und 1887 nahm er den Ruf nach Göttingen an, wo er bis zu seinem Tode das Institut für medizinische Chemie und Hygiene leitete. Es gelang ihm, zunächst die gröbsten baulichen Missstände zu beseitigen und im Oktober 1891 erfolgte der Umzug in den ersten Stock des inzwischen umgebauten ErnstAugust-Hospitals. ${ }^{229}$ Wolfhügels Interessenschwerpunkt lag auf der Hygiene und im Mai 1899 erreichte er, von seinem Lehrauftrag für medizinische Chemie entbunden zu werden. Das Argument, dass bereits sein Vorgänger Flügge nach dem Wintersemester 1884/85 keine Vorlesungen und Praktika in medizinischer Chemie mehr angekündigt hatte, hatte beim Ministerium verfangen. Sein Antrag, das Institut in „Hygiene-Institut" umzubenennen, wurde abgelehnt, 230 doch ist nach dem Wintersemester das Institut für medizinische Chemie und Hygiene unter dieser Bezeichnung nicht mehr im Vorlesungsverzeichnis belegt. ${ }^{231}$ Nach einer längeren Übergangsphase erfolgte zu Beginn des 20. Jahrhunderts dann auch in Göttingen die vollständige Eingliederung der medizinischen Chemie in die Physiologie, nachdem sich bereits zuvor die Hygiene aus der Verbindung gelöst und sich zum eigenständigen Spezialfach etabliert hatte.

Nachfolger Wolfhügels wurde Erwin von Esmarch (1855-1912), der zwölf Jahre die Leitung inne hatte und einige Umbauarbeiten, bedingt durch zunehmende bakteriologische Untersuchungen, erreichte. Dann folgten Hans Reichenbach 
(1864-1937) von 1911 bis 1934 und Franz Schütz (1887-1955) bis 1955, allerdings mit der Unterbrechung zwischen 1946 und 1950, als Schütz wegen seiner Verwicklung in die NS-Diktatur suspendiert war und durch Theodor Josef Bürgers (1881-1954) vertreten wurde. Während der Amtszeit von Schütz gelang im Januar 1939 der Kauf des Waisenkrankenhauskomplexes am Kreuzbergweg. Nach Umbauarbeiten konnte das Gebäude im Oktober 1940 bezogen werden und im Oktober 1941 wurde dann offiziell der Name von „Institut für medizinische Chemie und Hygiene" in „Hygienisches Institut" umbenannt. Schütz hatte einen entsprechenden Antrag u. a. damit begründet, dass das Institut rein hygienisch arbeitete und zugleich das letzte Institut in Deutschland sei, das noch diesen Doppelnamen trug. ${ }^{232}$

\section{Pharmakologie}

Die kunstgerechte Anwendung der Arzneimittel zählt seit jeher zu den wichtigsten Grundlagen ärztlichen Handelns. Durch die Medizinalordnung Friedrich II. von 1231, die die Trennung von Arzt und Apotheker bestimmte, wurde die Herstellung und Vergabe von Arzneimitteln in die Hand eines Spezialisten gelegt, des Apothekers. Indikation und Dosierung der Arznei war jedoch nicht allein Aufgabe des Apothekers, sondern auch des Arztes. Unter dem Einfluss der Naturwissenschaften im 19. Jahrhundert - vor allem der Chemie und experimentellen Physiologie - entwickelte die Medizin im Bereich der Pharmakotherapie experimentell gewonnene Methoden, die eine wissenschaftlich fundierte Nachprüfung der Heilmittelwirkung ermöglichten. Diese Grundlage führte dann zum Spezialfach der modernen experimentellen Pharmakologie.

In Göttingen wurde sie 1873 institutionalisiert. Unter der Leitung des außerordentlichen Professors Wilhelm Marmé (1832-1897), der zuvor am Physiologischen Institut tätig war, wurde im Wrisbergschen Haus eine entsprechende Abteilung eingerichtet. 1877 erfolgte der Umzug in das alte Auditorien-Gebäude, 1892 ins Ernst-August-Hospital und 1977 schließlich in das neue Universitätsklinikum. Göttingen bietet damit ein eindrucksvolles Beispiel, wie pharmakologische Institute mit geringem Aufwand in ältere bereits vorhandene Gebäude untergebracht wurden, die auf Grund gestiegener Anforderungen von anderen medizinischen Fächern verlassen worden waren. ${ }^{233}$ 



\section{Frauenstudium in Göttingen}

Die Möglichkeit, an der Göttinger Universität zu studieren, blieb Frauen bis zum Ende des 19. Jahrhunderts verwehrt, da sich zahlreiche Professoren immer wieder dagegen aussprachen.

Der Ursprung des Frauenstudiums in Göttingen liegt in anonymen Petitionen an das jeweilige Ministerium. Die erste Forderung erging 1747 an Friedrich den Großen, Frauen zum Studium zuzulassen. Diese wurde abgelehnt wie auch die folgenden. Immer wieder gelang es jedoch den Frauen, Privatvorlesungen zu besuchen und anschließend zu promovieren, wie Dorothea Schlözer (1770-1825), die 1787 lediglich aufgrund einer mündlichen Prüfung promoviert wurde, oder Charlotte Heidenreich von Siebold (1788-1859), der es mit Hilfe von Beziehungen 1817 gelang, eine Dissertation zu schreiben. Sie blieben jedoch Ausnahmen.

Später, Ende des 18. Jahrhunderts, folgten zahlreiche Anfragen, die allesamt abgelehnt wurden. Gegner des Frauenstudiums, wie Friedrich Benjamin Osiander, nahmen Stellung dazu, nannten jedoch keine Gründe, wieso Frauen das Studium verwehrt bleiben sollte. Osiander bestritt, „dass beim Unterricht characterloser Weiber und Mädchen viel Erfreuliches herauskomme. Sie sind wie ein Rohr, das der Wind bin und her wehet, und vollends zur Auctorschaft ganz verdorben. Das Schwangerwerden steht ibnen auf jeden Fall besser an, als über Scbwangerschaften zu schreiben. " 234

Nachdem in Zürich 1867 Frauen zum Studium zugelassen worden waren, wollten einige diesem Vorbild folgen. Befürworter waren in Göttingen Wilhelm Ebstein und der Ophthalmologe Hermann Schmidt-Rimpler (1838-1915), die sich dafür einsetzten, Frauen als Hörerinnen zu theoretischen Medizinvorlesungen zuzulassen. Was den praktischen Unterricht betraf, waren nur Kinder- und Frauenheilkunde erlaubt. ${ }^{235}$ Bedingung war, genügend Vorbildung zu besitzen und ein Maturitätsexamen abgelegt zu haben, um unter gleichen Voraussetzungen wie die Männer aufgenommen zu werden. Verfechter des Frauen-Medizinstudiums, wie Ludwig Meyer und Gustav Wolffhügel, behaupteten, dass die Vorbildung keinesfalls gewährleistet sei, da es keine Mädchengymnasien gab. Des Weiteren sei ein Studium nur möglich, wenn spezielle Frauenhochschulen existieren würden, ${ }^{236} \mathrm{da}$ der ,gemeinsame(n) Unterricht beider Geschlechter für einen scblimmen Mißstand (sorgen würde), (...) weil es vermieden werden sollte, kranke Männer vor Damen zu demonstrieren. Die weiblichen Ärzte wollen doch keine Männer behandeln. " 237 


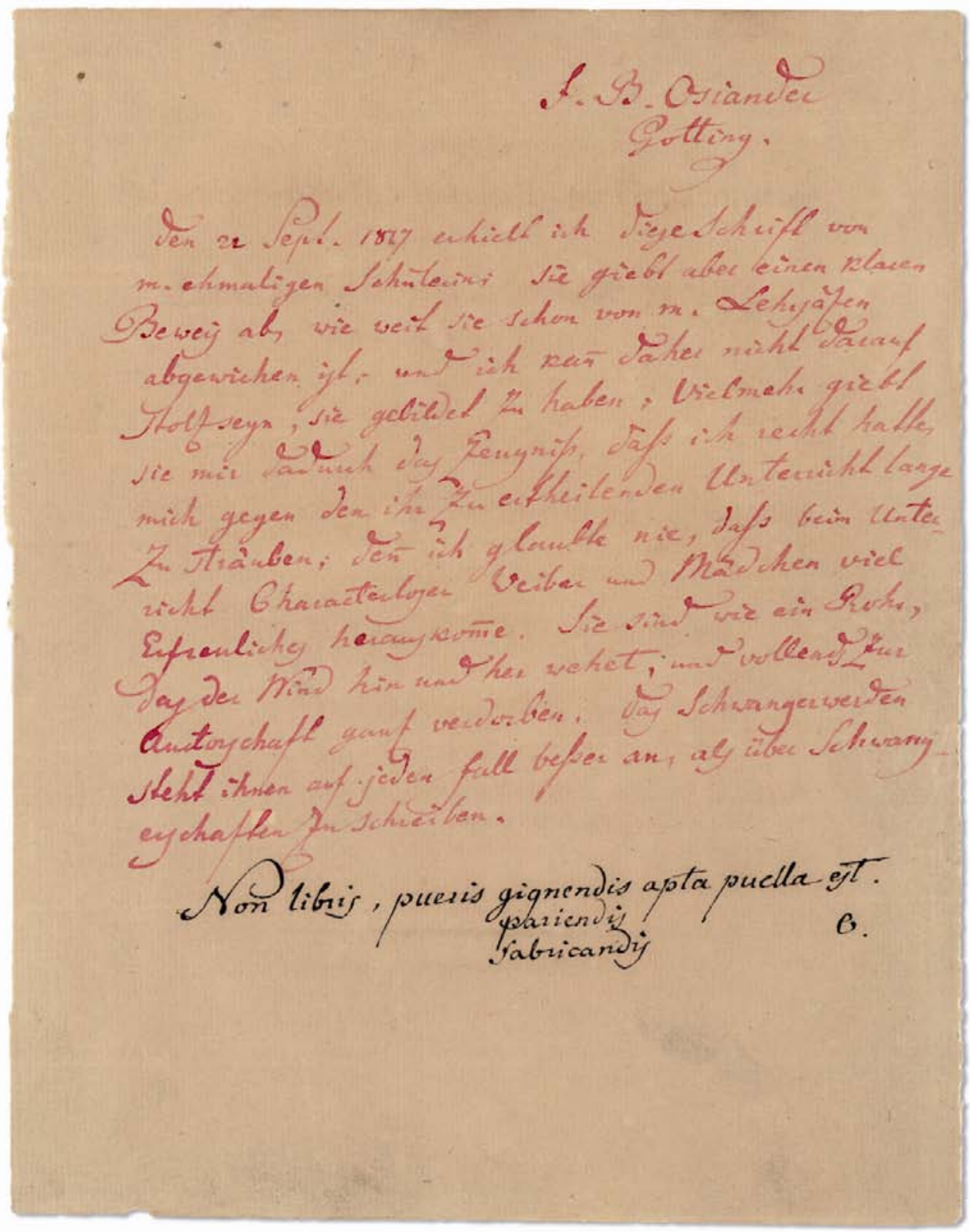

Abb. 32: Brief Osianders zum Frauenstudium 
Die praktische Umsetzung unterblieb hingegen, da trotz der erfüllten Bedingungen Frauen aufgrund der Geschlechterhierarchie keine Chancengleichheit gewährleistet wurde. Orth äußerte und sprach dabei für alle Gegner des Frauenstudiums: „Aber auch für den Fall, daß die Vorbildung nachgenviesen werden könnte, müßte ich aus theoretischen wie praktischen Gründen gegen die Zulassung von weiblichen Zubörerinnen, mag es sich um rite aufgenommene Studierende oder um Gastbörerinnen handeln, mich aussprechen müssen. " 238

Das Verbot der Zulassung von Frauen wurde vom Anfang des 20. Jahrhunderts an immer großzügiger gehandhabt. So sprachen sich mehrere Fakultäten für eine Immatrikulation von Frauen aus. Folglich wurden immer mehr Gasthörerinnen zugelassen. Auch die Zahl der Hörerinnen an der Medizinischen Fakultät stieg. Nach zahlreichen Petitionen folgte am 18. August 1908 ein Erlass, der das Frauenstudium in Göttingen offiziell autorisierte. 239 



\section{Die Medizinische Fakultät während der NS-Diktatur}

Als die Nationalsozialisten 1933 an die Macht kamen, konnten sie in Göttingen auf eine seit dem Frühjahr 1922 bestehende Parteiorganisation und auf ein überdurchschnittliches Anhängerpotenzial innerhalb der Bevölkerung zurückgreifen. Im Juli 1932 hatten 51 \% der Göttinger Bevölkerung die NSDAP gewählt, im Reich waren es nur gut $37 \%$ gewesen. ${ }^{240}$

\section{Gleichschaltung und Entlassungspolitik}

In der Medizinischen Fakultät war der Gynäkologe Professor Heinrich Martius der einzige „Nichtarier" unter den insgesamt 17 amtierenden Ordinarien und beamteten außerordentlichen Professoren. Fünf der 15 nichtbeamteten außerordentlichen Professoren und einer der 23 Privatdozenten konnten keinen ,arischen Stammbaum" nachweisen. ${ }^{241}$ Insgesamt entsprach dies einer Größenordnung von rund 12\%; das Presseamt der Universität hingegen gab den „Grad der Verjudung der Georgia-Augusta" in der Medizinischen Fakultät mit insgesamt $11 \%$ an. ${ }^{242}$ Bereits im Herbst 1934, also noch vor dem Erscheinen des Reichsbürgergesetzes im Jahre 1935, waren - mit Ausnahme von Heinrich Martius und Rudolf Ehrenberg (18841969) - alle jüdischen Dozenten aus der Medizinischen Fakultät vertrieben.

Das Schicksal des jüdischen Physiologen Rudolf Ehrenberg hingegen dokumentiert, dass Zivilcourage in der Frühphase der nationalsozialistischen Herrschaft einiges bewirken konnte. Der Dekan Hans Beumer hatte Ehrenberg nahegelegt, im Sommersemester 1933 auf seine Lehrtätigkeit zu verzichten. Am 2. Mai 1933 intervenierte daraufhin der Direktor des Physiologischen Instituts, Hermann Rein (1898-1953), bei Beumer mit der Begründung, Ehrenberg sei mit der Vorlesung und dem Praktikum der Physiologischen Chemie beauftragt und sein Ausfall daher keinesfalls zu verantworten. Im Übrigen sei er „Langemarck-Kämpfer“. Bereits am folgenden Tag zog der Dekan seine Empfehlung wieder zurück. ${ }^{243}$ Der Minister gab dem Antrag des Physiologischen Instituts auf Weiterbeschäftigung zunächst statt, ordnete aber zum 30. September 1935 Ehrenbergs Entlassung an. Da Ehrenberg die Venia Legendi behielt, konnte er auch in den folgenden Semestern seine Lehrtätigkeit freiwillig ausüben. Erst die Anfrage des neugewählten Dekans 
Walther Krantz vom 27. Mai 1938, ob Ehrenberg aufgrund der verschärften Promotionsbedingungen überhaupt noch die Lehrbefugnis ausüben dürfe, führte nunmehr zu ihrem Entzug. ${ }^{244}$

Von den Dozenten mit ,jüdischer Abstammung “ blieb also nur der Leiter der Frauenklinik, Heinrich Martius, der nach der damaligen Terminologie als „Mischling zweiten Grades" eingestuft wurde, während der gesamten NS-Diktatur an der Universität Göttingen im Amt. Nicht so eindeutig lassen sich die eigentlichen Ursachen der politisch bedingten Entlassungen nachzeichnen. In Göttingen sind zwei entsprechende Fälle belegt. Es handelt sich dabei um den Privatdozenten für Gynäkologie Robert Brühl (1898-1976)245 und den Privatdozenten für Kinderheilkunde Hugo Fasold (1896-1975). ${ }^{246}$

\section{Karl Saller und die Einrichtung eines „Lehrstuh/s für Rassenhygiene"}

Die Auseinandersetzungen um Karl Saller (1902-1969) und die geplante Errichtung eines „Lehrstubls für Rassenhygiene“ dokumentieren augenfällig die Situation innerhalb der Medizinischen Fakultät während der NS-Diktatur. ${ }^{247}$ Kein anderes Fach wurde von den nationalsozialistischen Machthabern so aufgewertet und gleichzeitig so missbraucht wie die Rassenhygiene. Göttingen zählt zu den wenigen deutschen Universitäten, an denen diese Fachrichtung bis zum Ende der nationalsozialistischen Zwangsherrschaft nicht institutionalisiert war.

Sallers Schwierigkeiten, die ihm seit 1933 auch innerhalb der Medizinischen Fakultät zusehends erwuchsen, beruhten im Wesentlichen auf seinen rassenhygienischen Thesen. Sein ,dynamischer Rassebegriff“ ließ sich schwerlich in Einklang mit der damals herrschenden engstirnigen Rassenlehre bringen. Eng mit Sallers Schicksal verknüpft war die Errichtung einer Professur für Rassenhygiene. Im Juni 1934 lag der Fakultät die Genehmigung zur Errichtung einer entsprechenden Professur vor. Um Saller, dessen Chancen aufgrund seiner wissenschaftlichen Qualifikation gut waren, auf jeden Fall zu verhindern, übte der örtliche NS-Dozentenbund enormen Druck auf die Fakultät aus und das Reichs- und Preußische Ministerium für Wissenschaft entzog Saller am 14. Januar 1935 gemäß $\ 18$ der Reichshabilitationsordnung die Lehrbefugnis. ${ }^{248}$ Das politisch bedingte Berufsverbot des favorisierten Kandidaten Saller bereitete auch der Medizinischen Fakultät bei der Besetzung des geplanten Lehrstuhls für Rassenhygiene große Probleme. Da andere Universitäten ebenfalls entsprechende Lehrstühle einrichteten, waren qualifizierte Wissenschaftler selten. Im Frühjahr 1942 schließlich zog das Ministerium seine Mittelzusage zur Einrichtung eines „Lehrstubls für Vererbungswissenschaft" an der Universität Göttingen endgültig zurück. ${ }^{249}$ 
Hatte die Fakultät im Falle Saller sich des politischen Drucks nicht erwehren können, so gelang es ihr, bei der Errichtung und Besetzung der „Professur für Ras-

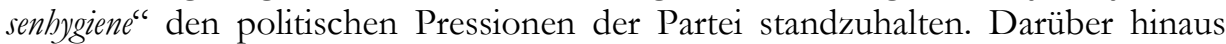
verhinderte sie eine Senkung des wissenschaftlichen Niveaus zugunsten parteipolitischer Überzeugung. Der Verlust einer Professur war freilich als Preis hoch, aber im Hinblick auf die Würde und wissenschaftliche Selbstachtung der Medizinischen Fakultät gerechtfertigt.

\section{Zwangssterilisationen an Universitätskliniken}

Am 1. Januar 1934 trat das „Gesetz zur Verbütung erbkranken Nachwuchses“ in Kraft. Für die Durchführung des Eingriffs waren die Universitätsfrauenklinik und die Chirurgie zuständig. In der Göttinger Frauenklinik wurden insgesamt 787 Frauen zwangssterilisiert, ${ }^{250} 60 \%$ allein während der ersten drei Jahre. An der Spitze der Indikationen stand ,angeborener Schwachsinn“ mit rund $60 \%$. An zweiter Stelle folgte die „Schizophrenie“ mit 22\%. Zehn Frauen wurden ohne Angabe einer Indikation sterilisiert. Zwei Drittel aller Betroffenen gehörten sozialen Unterschichten an (Dienstmädchen, Hausangestellte, Arbeiterinnen); nur $5 \%$ besaßen höhere Schulbildung (Lehrerinnen, Studentinnen, Bankangestellte). Postoperative Komplikationen traten nur in geringem Maße auf. Gestorben sind insgesamt drei sterilisierte Frauen, das entspricht einer Letalitätsrate von unter $1 \%$. Diese nüchternen statistischen Angaben zum operativen Eingriff dürfen jedoch nicht darüber hinwegtäuschen, dass hinter jedem Fall ein Mensch steht, dem durch die Operation psychische und physische Dauerschäden zugefügt wurden.

Über die Anzahl der Zwangssterilisationen in der chirurgischen Klinik lassen sich keine endgültigen Aussagen machen, da die entsprechenden Krankenakten der Jahre 1934 und 1936 nicht mehr aufzufinden sind. Aufgrund der Auswertung der Jahrgänge 1935 und 1937 bis 1945 lässt sich im Vergleich folgendes Bild konturieren. Insgesamt wurden in Göttingen mehr Männer (rund 52 \%) als Frauen (knapp $48 \%$ ) sterilisiert. Die Göttinger Zahlen entsprechen ziemlich exakt den Daten, die sich auf das gesamte Reich beziehen. ${ }^{251}$ Im Hinblick auf die jeweils gestellte Diagnose zeigen sich geschlechtsspezifische Unterschiede. So wurden mehr Männer aufgrund von „Schizophrenie“ und deutlich weniger wegen ,angeborenem Schwachsinn" sterilisiert. Vergleichsweise hoch ist bei den Männern der Anteil mit der Diagnose „schwerer Alkoholismus“.252 


\section{Gottfried Ewald und die Auswirkungen der Euthanasie in Göttingen}

Nachdem durch den Runderlass des Reichsinnenministeriums vom 18. August 1939 die Vernichtung behinderter Kinder bereits beschlossen worden war, bot der Ausbruch des zweiten Weltkrieges den äußeren Anlass, die bereits lange vorher geplante und vorbereitete systematische Vernichtung „lebensunwerten Lebens " zu beginnen.

Die Heil- und Pflegeanstalten hatten für jeden Patienten innerhalb einer vorgegebenen Frist einen Meldebogen auszufüllen, wobei vor allem der Grad der Erkrankung und die Beschäftigungsmöglichkeit differenziert angegeben werden mussten. Die Auswertung erfolgte in der Berliner Euthanasie-Zentrale, die sich in der Tiergartenstraße 4 befand und der Aktion den Namen T 4 gab. Am 5. Juli 1940 kamen diese Meldebögen auch in die Heil- und Pflegeanstalt Göttingen. Zur Beantwortung wurde eine Frist bis zum 1. August 1940 gesetzt. ${ }^{253}$ Die Euthanasiemaßnahmen wurden in Göttingen dann 1941 durchgeführt. Vorausgegangen war bereits eine Aktion zur Vernichtung geisteskranker Juden. Der Transport von 12 jüdischen Patienten erfolgte am 21. September 1940. ${ }^{254}$ Der Abtransport der nichtjüdischen Geisteskranken begann am 11. März 1941. Insgesamt fielen durch die vier Transporte 238 Patienten aus der Göttinger Heil- und Pflegeanstalt der Euthanasie zum Opfer. 129 zum Transport Vorgesehene konnten vom Leiter der Anstalt, Gottfried Ewald, durch Zurückstellung gerettet werden.255

Ewald war es auch, der den Mut hatte, gegen die verbrecherischen Euthanasiemaßnahmen öffentlich zu protestieren. Das erscheint auf den ersten Blick erstaunlich, da er politisch mit den damaligen Machthabern durchaus sympathisierte und auch Positionen seines wissenschaftlichen Denkens, vor allem im Bereich der Eugenik, in Einklang mit der nationalsozialistischen Weltanschauung standen. Ewald gehörte mit seiner christlich-konservativen Gesinnung zu jenen Deutschen, die Hitlers Machtantritt hoffnungsvoll begrüßt hatten. In einem Gutachten des Göttinger NS-Dozentenbundes von 1939 wurde Ewald als ,positiv zum Dritten Reich stehend" eingestuft. ${ }^{256}$ In Ewalds politischer und wissenschaftlicher Haltung dürfte der Grund zu suchen sein, warum ihn die Berliner Euthanasieverantwortlichen als Gutachter bei der Auswertung der Fragebögen zu gewinnen suchten. Mit dem Schreiben vom 8. August 1940 wurde er in seiner Funktion als Ordinarius der Psychiatrischen Nervenklinik der Universität Göttingen vom Leiter der Reichsarbeitsgemeinschaft Heil- und Pflegeanstalten, Professor Werner Heyde (1902-1964), „zur Erörterung dringender kriegswichtiger Maßnabmen auf dem Gebiete des Heil- und Pflegewesens" für den 15. August 1940 nach Berlin in die Tiergartenstraße 4 eingeladen. ${ }^{257}$ Ewald hielt den Verlauf dieser Sitzung fest. Als Heyde die Meinung vertrat, auch senile und tuberkulöse Kranke kämen für die Euthanasie in Betracht, 
habe er Einspruch dagegen erhoben und klar zum Ausdruck gebracht, dass er eine Gutachtertätigkeit in dem vorgesehenen Rahmen ablehne. ${ }^{258}$

Nach Göttingen zurückgekehrt, begründete Ewald mit einer Fülle von Argumenten umgehend seine ablehnende Haltung, wobei er bei seinem Votum davon ausging, dass ein Gesetz zur Vernichtung „lebensunwerten Lebens" in Vorbereitung sei, wie ihm auch in Berlin vorgespiegelt wurde.

Gottfried Ewald, der im August 1940 direkt und mutig seine Stimme gegen die Euthanasie erhob, zählt zu den ganz wenigen deutschen Ärzten, die persönlich und beruflich Widerstand gegen dieses medizinische Verbrechen leisteten.

\section{Der „Fal/“ Hermann Rein}

Hermann Rein, der im Sommersemester 1932 als ordentlicher Professor der Physiologie nach Göttingen berufen worden war, schuf innerhalb kürzester Zeit aus dem inzwischen bedeutungslos gewordenen Physiologischen Institut eine produktive und weltweit anerkannte Lehr- und Forschungsstätte. Politisch gefordert wurde Rein erstmals im Zusammenhang mit den Schwierigkeiten seines ,nichtarischen "Mitarbeiters Ehrenberg. Diese Haltung ist ein beredtes Zeugnis für Reins Distanz zu den damaligen Machthabern. Obwohl er mehreren Gliederungen der Partei angehörte und seit dem 1. März 1934 auch förderndes Mitglied der SS war, fiel seine Beurteilung durch die Partei insgesamt recht ungünstig aus. ${ }^{259}$

Trotz seiner ambivalenten Haltung zum NS-System blieben Rein und das Physiologische Institut von politischen Angriffen und Auseinandersetzungen - sieht man von dem Fall Ehrenberg einmal ab - während der gesamten Naziherrschaft verschont. Zum einen lag die Ursache dafür im internationalen Ansehen, das Rein als Wissenschaftler genoss, zum anderen in seinen Verbindungen, die er im Zusammenhang mit seinen Tätigkeiten in der Luftfahrtmedizin knüpfen konnte. Im März 1935 erhielt er vom Reichsminister für Wissenschaft, Erziehung und Volksbildung einen Lehrauftrag für Luftfahrtmedizin, 1937 erfolgte die Ernennung zum ordentlichen Mitglied der Akademie für Luftfahrtforschung. ${ }^{260}$

Vor allem die im Auftrage des Luftfahrtministeriums durchgeführten Forschungen schufen Rein den nötigen Freiraum gegenüber dem örtlichen NSDozentenbund. Und gerade diese Konstellation offenbart die Problematik des Wissenschaftlers unter einer Diktatur. Rein trat als Präsident der Göttinger Akademie der Wissenschaften und als Vorsitzender der Physiologischen Gesellschaft immer wieder entschieden für eine Forschung ein, die frei von aller ideologischen Bindung sein müsse, übersah dabei aber offensichtlich, dass in einer Gewaltherrschaft gerade Forschungsergebnisse mit einem solch ethischen Postulat dem Missbrauch besonders leicht ausgesetzt sind. 


\section{Rudolf Stich - Dekan der Medizinischen Fakultät während des Zweiten Weltkriegs}

Rudolf Stich (1875-1960), der 1911 auf den Lehrstuhl für Chirurgie nach Göttingen berufen wurde, spielte während der NS-Zeit an der Georg-August-Universität eine zentrale Rolle. Vom Wintersemester 1939/40 bis 1945 war er Dekan der Medizinischen Fakultät und übte dieses Amt ganz im Sinne der damaligen Machthaber aus. Seine Unterstützung und Verteidigung des Gesetzes zur Verhütung erbkranken Nachwuchses zeigt Stich als einen Befürworter der NSGesundheitspolitik. Entsprechend führte er in seiner Klinik Zwangssterilisationen aus. Stichs Zustimmung zur NS-Gesundheitspolitik traf jedoch nicht auf alle Bereiche zu. Die Euthanasie lehnte er entschieden ab, vertrat diese Meinung auch in der Öffentlichkeit und half durch seine Unterstützung des in diesem Zusammenhang in Schwierigkeiten geratenen Göttinger Neurologen und Psychiaters Gottfried Ewald. Kurz vor Kriegsende, am 13. April 1945, übergab Stich das Dekanat an Rudolf Schoen (1892-1979), führte jedoch die Leitung der Chirurgischen Klinik weiter. Am 13. Juni 1945 wurde er als ehemaliger Dekan verhaftet, kam zunächst nach Hildesheim ins Gefängnis und von dort in das Lager Westertimke. Nach seiner Entlassung am 31. August 1945 beantragte Stich seine Emeritierung, der am 20. September 1945 rückwirkend zum 1. September stattgegeben wurde. Stich kam so seiner Entlassung zuvor, da er sich als Emeritus nicht dem Entnazifizierungsausschuss stellen musste. ${ }^{261}$

Die Medizinische Fakultät spiegelt die typischen Merkmale der NS-Zeit wider. Als Abbild der gesamten Universität schwankt sie zwischen „Selbst-Gleichschaltung“, Gehorsam und ansatzweisem punktuellem Widerspruch. Wie schwer es für sie war, sich mit den Naziverbrechen auseinanderzusetzen, zeigte ihre Haltung zu Beginn des Nürnberger Ärzteprozesses. In einer Stellungnahme vom 19. November 1947 formulierte der Dekan Hans Joachim Deuticke (1898-1976):

„,...] Auch unsere biesige Fakultät hofft, dass durch den bevorstehenden Prozess in Nürnberg gegen deutsche Aerżte geklärt wird, dass nur eine verschwindend geringe Zabl von Aeraten, die in eigener Verantwortung handelten, sich scbuldig gemacht hat und dem gemäss bestraft werden muss, dass aber die deutsche Aeryteschaft als solche entsprechend ibrer Tradition und ibrer inneren Ueberzengung frei von Schuld und nicht mit Vorwürfen zu belasten ist [...]. "262

Die Göttinger Medizinische Fakultät lehnte also a priori eine Kollektivschuld der deutschen Ärzte ab. Verbrechen hätten nur eine „verschwindend geringe Zabl von Aeraten, die in eigener Verantwortung bandelten“ begangen. Die „deutsche Aerateschaft als solche" hingegen sei frei von Schuld. 


\section{Zwangsarbeiter in den Universitätskliniken}

Während des Zweiten Weltkrieges wurde die deutsche Kriegswirtschaft zum Großteil durch den massiven Einsatz von Zwangsarbeitenden aufrechterhalten. Bereits ab 1939 wurden ausländische „Zivilarbeiter“, im damaligen Sprachgebrauch „Fremdarbeiter" genannt, zum Arbeitseinsatz nach Deutschland gebracht. Spätestens seit 1941 war die deutsche Kriegswirtschaft infolge der Einberufungswellen und Erhöhung der Rüstungsproduktion substanziell auf ausländische Arbeitskräfte angewiesen. Verantwortlich für ihren zentral gesteuerten und organisierten Einsatz war seit dem 21.März 1942 Fritz Sauckel (1894-1946), der von Hitler zum „Generalbevollmächtigten für den Arbeitseinsatz“ ernannt worden war. Von Zwangsarbeit betroffen oder bedroht waren Juden, Häftlinge der Konzentrationslager, Kriegsgefangene und „Zivilarbeiter". Ausländische Kriegsgefangene aus Italien, Polen, der Sowjetunion und Frankreich wurden häufig in den Status der „Zivilarbeiter" überführt. Für den Zeitraum von 1940 bis 1945 wurden an der Medizinischen Fakultät über 120 Zwangsarbeitende ermittelt, die aus ganz Europa, überwiegend aus Russland, Polen, der Ukraine, Frankreich und den Niederlanden kamen. Fast die Hälfte der Zwangsarbeitenden waren zwischen 18 und 21 Jahre alt. Die „Einstellung“ erfolgte hauptsächlich in den Jahren 1942 und 1943. Sie waren vor allem in den Abteilungen der Chirurgie, Inneren und Gynäkologie eingesetzt, arbeiteten aber auch in der Nervenklinik, Hautklinik, der HNO- und Kinderklinik sowie in der Zahnmedizin und schließlich innerhalb der Mikrobiologie, Pathologie und Anatomie. Im nicht-klinischen Bereich verfügten die Küche, die Wäscherei, das Maschinenhaus sowie die Gärtnerei über Zwangsarbeitende. Während die Zwangsarbeiterinnen überwiegend als Reinigungskräfte und Küchenhilfen eingesetzt wurden, waren z. B. französische und niederländische Medizinstudierende auch in der Pflege beschäftigt. Belegt ist auch der Einsatz von „notdienstverpflichteten “ Ärzten. ${ }^{263}$ Zum Gedenken an die Zwangsarbeiterinnen und Zwangsarbeiter hat die Medizinische Fakultät am 8. Mai 2008 vor dem Gebäude der alten Frauenklinik in der Humboldtallee 19 einen Gedenkstein errichtet. Anknüpfend an das Cicero Zitat „,ui dolet, meminit“ aus der Rede „Pro Murena“ 264 hat er den folgenden Text: 


\section{Ihr Leiden verwehrt Vergessen}

Zum Gedenken an die Zwangsarbeiterinnen

und Zwangsarbeiter der Göttinger Universitätskliniken

Von 1939 bis 1945 wurden zahlreiche Frauen und Männer an den

Kliniken der Universität Göttingen zur Zwangsarbeit eingesetzt.

Sie lebten und arbeiteten unter unwürdigen Bedingungen.

Viele Zwangsarbeiterinnen und Zwangsarbeiter wurden zudem

in den Abteilungen der Kliniken untersucht und behandelt.

Eine große Zahl blieb medizinisch unterversorgt

und wurde zu medizinischen Zwecken missbraucht.

Wir verneigen uns vor den Opfern 


\section{Sammlungen der Medizinischen Fakultät}

Vier berühmte Sammlungen befinden sich im Besitz der Medizinischen Fakultät und bieten Forschern und Interessierten aus aller Welt wichtiges Forschungs- und Anschauungsmaterial. Als erstes ist zu nennen die „Humanembryologische Dokumentationssammlung Blechschmidt" der Abteilung Embryologie des Zentrums Anatomie, zweitens die kulturgeschichtliche Sammlung von Heinz Kirchhoff mit dem Namen "Symbole des Weiblichen“", drittens die „Sammlung zur Geschichte der Geburtshilfe" im Institut für Ethik und Geschichte der Medizin und schließlich die „Blumenbachsche Schädelsammlung"in der Göttinger Anatomie.

\section{Die "Humanembryologische Dokumentationssammlung}

\section{Blechschmidt"der Abteilung Embryologie des Zentrums Anatomie}

Im Zentrum Anatomie befinden sich in 64 Schaukästen räumliche Rekonstruktionen von ca. $75 \mathrm{~cm}$ Größe, die Lage- und Formcharakteristika der Organe menschlicher Embryonen in 50-200 facher Vergrößerung veranschaulichen. Besonderes Anliegen Erich Blechschmidts (1904-1992) war es, die Entwicklungsstadien der embryonalen Organe nicht isoliert und abstrakt zu präsentieren, sondern ihre Lage, Form und Struktur in ihrem natürlichen räumlichen Umfeld im Organismus begreifbar zu machen. Dazu entwickelte er ein besonderes Verfahren und stellte von 1950 bis 1972 aus 200000 Einzelpräparaten histologischer Schnittserien menschlicher Embryonen der ersten acht Schwangerschaftswochen diese Rekonstruktionen zusammen. Die zehn Mikrometer dünnen Schnitte, die eingefärbt unter dem Mikroskop untersucht werden können, wurden in viele zweidimensionale Schnitte zerlegt, aus denen dann dreidimensionale Präparate rekonstruiert werden konnten. Diese vermitteln anschaulich das Bild eines Embryos, seiner Organe und ihrer Lagebeziehungen. Die Rekonstruktionen sind weitaus klarer als graphische, seien es manuelle oder computergestützte.

Diese Sammlung ist weltweit einmalig und genießt internationales Ansehen als Forschungsobjekt. ${ }^{265}$ 

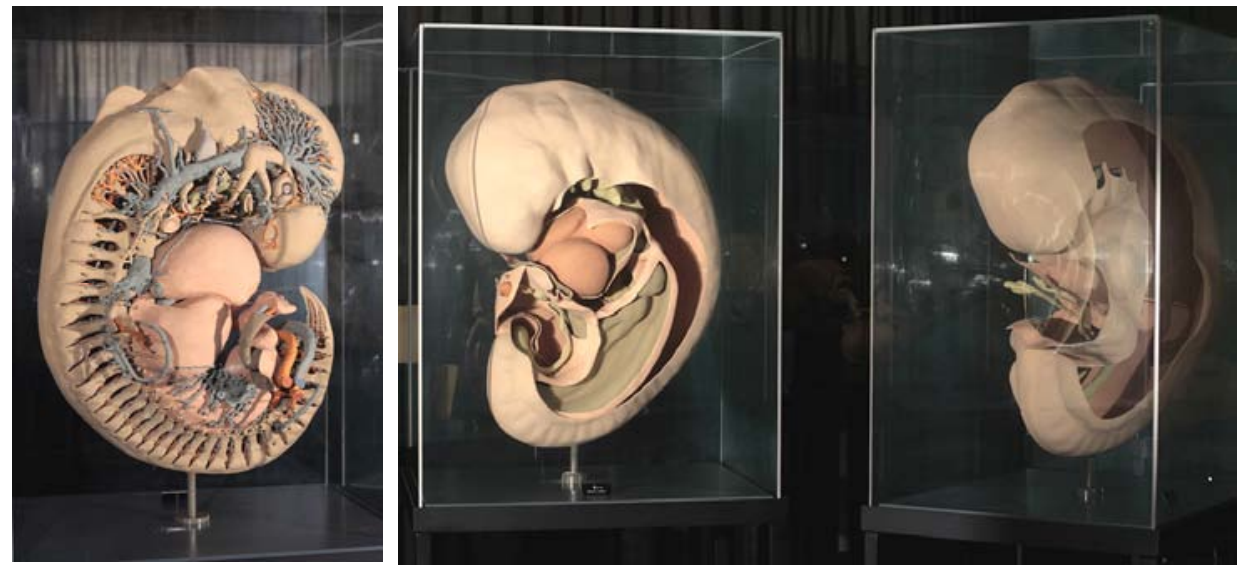

Abb. 33: Rekonstruktion eines zweimonatigen menschlichen Embryos

\section{"Symbole des Weiblichen".}

\section{Die kulturgeschichtliche Sammlung Heinz Kirchhoff}

Auch die kulturgeschichtliche Sammlung Heinz Kirchhoffs „Symbole des Weiblichen“ mit über 650 Objekten, von denen sich seit 1997200 Exponate als Dauerausstellung in den Schaukästen der Kleinen Galerie im Universitätsklinikum befinden, ist herausragend.

Kirchhoff (1905-1997) schenkte die jahrzehntelang in unterschiedlichen Kulturkreisen gesammelten Objekte aus Anlass seines 80. Geburtstages 1985 der Medizinischen Fakultät. Der Aufbau dieser Sammlung, die er während seiner Tätigkeit als Direktor der Universitätsfrauenklinik zusammenstellen konnte, wurde auch durch Spenden unterschiedlicher Provenienz ermöglicht.

Die Exponate demonstrieren in einzigartiger Weise sinnfällig kulturhistorische Zusammenhänge der Symbole des Weiblichen, der Fruchtbarkeit, der Mutterschaft und Geburt und der Rolle der Frau in unterschiedlichen Kulturen und Zeiten und verdeutlichen ihre Funktion nicht nur im alltäglichen Leben, sondern auch ihre Bedeutung in den Schöpfungsmythen.

Die Sammlung besteht laut Kirchhoff zur Hälfte aus Originalen, die er von Reisen und internationalen Kongressen in aller Welt mitgebracht hat. In Afrika, Asien, Mittel- und Südamerika konnte er zudem Kontakte zu Museen knüpfen, die ihm die Möglichkeit gaben, Kopien sehr alter Originale anzufertigen, was allerdings oftmals mit Schwierigkeiten verbunden war, da die Direktoren befürchteten, dass ihre wertvollen und unersetzlichen Objekte beschädigt werden könnten.

Der Anstoß, Muttergottheiten zu sammeln, erwuchs zunächst aus dem ästhetischen Bedürfnis Kirchhoffs, der von ihrer Schönheit fasziniert war. Eine andere 
Motivation bezog sich später auf die ethische Bedeutung der Rolle der Frau. Die Würdigung ihrer Stellung in der Kulturgeschichte der Menschheit führte zur Gleichachtung und Gleichstellung sowie zur Partnerschaft, der die Zukunft gehört.

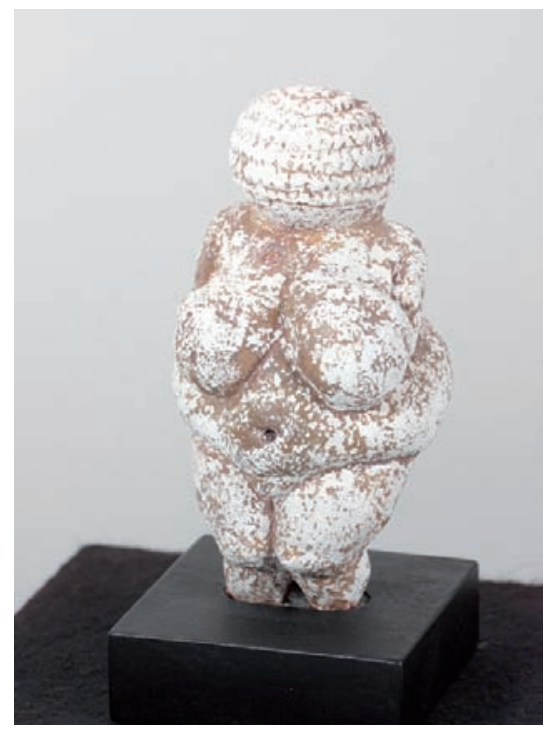

Abb. 34: Nachbildung der „Venus“von Willendorf (Österreich) ca. 25000 v. Chr.

Die Ausstellung gewährt einen systematischen Einblick in die Bedeutung von Mutterschaft, Fruchtbarkeitsmythen, Fortpflanzung, Schwangerschaft und Geburt sowie sakralen und profanen Ritualen in unterschiedlichen Kulturkreisen und Zeiten. Interessant sind besonders die Parallelen, die sich bei der Betrachtung altsteinzeitlicher Muttergottheiten und moderner Darstellungen auftun. So lassen sich deutliche Gemeinsamkeiten feststellen in der Figur der „Black Venus “von Niki de St. Phalle (1930-2002) und einer altsteinzeitlichen Venus, der Venus von Willendorf. In beiden werden Bauch, Becken und Brüste überdimensioniert, während der Kopf unbedeutend und ohne individuelle Züge ist.

Eine Vitrine enthält Votivgaben als Bitte oder Dank für Kindersegen. Gebäckformen (Model) für Hochzeitsgebäck, besonders aus dem 17. und 18. Jahrhundert, spiegeln in den Symbolen von Storch, Granatapfel, Hebamme und Herz Segenswünsche für das Brautpaar für Kinderreichtum. Auch Tier- und Pflanzensymbole lassen den Wunsch nach Fruchtbarkeit und Fortpflanzung erkennen.

Drei Schaukästen zeigen Darstellungen aus dem griechisch- römischen Bereich. Hervorzuheben ist eine Artemisstatue. Als Göttin der Fruchtbarkeit und der Jagd veranschaulicht sie sinnfällig Entstehung und Ende des Lebens. 
Auch die vier Vitrinen, die den afrikanischen Muttergottheiten gewidmet sind, verdeutlichen in den Holzplastiken der Ahnen die bedeutungsvolle Aufgabe der Frau, Leben hervorzubringen und zu erhalten sowie die Verbindung zu den Vorfahren herzustellen. Sie symbolisieren daher den ewigen Kreislauf von Entstehen und Vergehen. Ausgestellt sind außer den Kultobjekten (Götter, Dämonen, Ahnen) Embleme der Macht und des Prestiges, deren Bedeutung aber nicht immer erklärt werden kann, da die Objekte manchmal über Händler erworben wurden. Sogar Wissenschaftler teilten oftmals ihre Kenntnisse der Zusammenhänge aus religiösen Gründen nicht mit. Besonders hervorgehoben werden müssen die sogenannten Goldstaubgewichte der Ashanti aus Ghana. Sie waren die offizielle Währungseinheit, bis Ghana 1912 das englische Pfund einführen musste. Es sind im Wachsausschmelzverfahren hergestellte Figuren oder Figurengruppen, die das alltägliche Leben in jenem Kulturkreis spiegeln, z.B. Geburtsszenen.

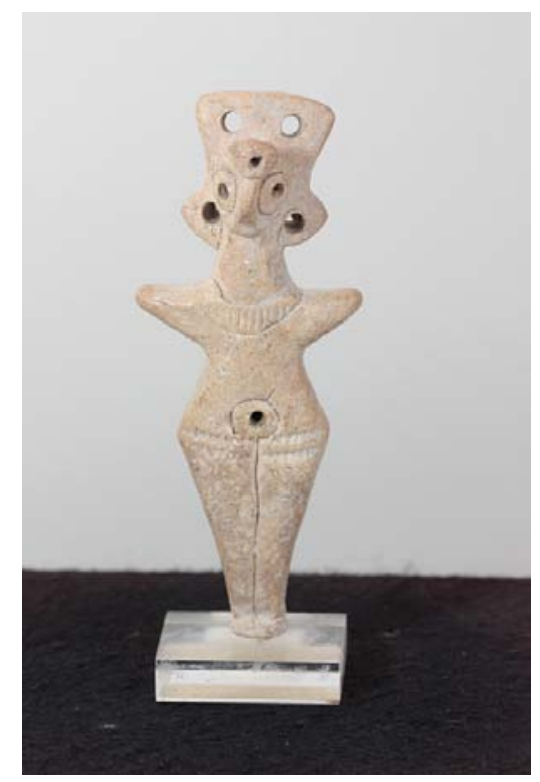

Abb. 35: Weibliche Figur (Nordsyrien), ca. 2000 v. Chr.

Eine Vitrine gilt den Plastiken aus dem südostasiatischen Gebiet, in dem unterschiedliche Religionen aufeinandertreffen (Hinduismus, Buddhismus, Islam, Christentum und Naturreligionen) und das Alltagsgeschehen prägen. Die thailändischen Mutterschaftsfiguren, wie jene aus Sawankhalok, dienten der Abwehr von Dämonen. Sie wurden enthauptet und den Hochschwangeren bzw. Gebärenden in das Bett gelegt. Nach einer glücklichen Entbindung wurde ihnen dann der Kopf wieder aufgesetzt. 
Ein weiterer Schaukasten zeigt weibliche Ahnenfiguren aus Papua-Neuguinea, die davon künden, dass dort in der Vorzeit eindeutig matriarchalische Zustände herrschten. Die Plastiken tradieren Ursprungsmythen, die ein harmonisches Weltbild des Miteinanders bezeugen.

Die Präsentation wird durch vier Vitrinen mit Skulpturen aus Mittel- und Südamerika abgerundet. Viele der alten Objekte wurden in Schachtgräbern entdeckt und sind gut erhalten. Es kann vermutet werden, dass diese Grabbeigaben den Toten an das Leben erinnern oder den Neubeginn in einer anderen, neuen Welt versinnbildlichen sollten. Gedeutet wurden sie auch als Spielzeugpuppen. Die Formgebung dieser Statuetten gemahnt an die altzeitlichen Fruchtbarkeitsfiguren mit ihrer Überbetonung der Beckenpartie. Touristen erwerben Kopien dieser Puppen als Souvenir.

Da von den ca. 650 Objekten, die Heinz Kirchhoff gesammelt hat, in der Dauerausstellung nur 200 Exponate ständig betrachtet werden können, hat der Förderverein Sammlung Heinz Kirchhoff e.V. auch 51 Exponate vorbereitet, die für Wanderausstellungen angefordert werden können. Durch sie ist ebenfalls eine kulturgeschichtliche Reise von der Altsteinzeit bis in die Neuzeit durch mehrere Kulturen möglich. ${ }^{266}$

\section{"Metalla ferri. optumo pessimoque vitae instrumento". Die Sammlung zur Geschichte der Geburtshilfe}

Die Sammlung zur Geschichte der Geburtshilfe ist wohl die älteste der Sammlungen, da sie seit dem späten 18. Jahrhundert besteht. Das Sammeln von Objekten galt ab dem 17. und 18. Jahrhundert als eine wichtige Methode der Wissensverarbeitung und bildet die Basis für Museen und Kabinette. Sammelten zunächst die anatomischen Institute Objekte, so legten dann später viele der sich ausdifferenzierenden Spezialdisziplinen großen Wert auf Sammlungen.

Die „Königliche Entbindungsanstalt“ der Universität Göttingen konnte seit 1792 eine derartige Sammlung, die von Johann Heinrich Fischer initiiert worden war und der sie bei seinem Abschied 1792 dem Entbindungshaus schenkte, aufweisen.

Friedrich Benjamin Osiander, der die Entbindungsanstalt übernahm, fertigte dann eine Aufstellung der Bestände des Kabinetts an, die 20 knöcherne und 20 in Weingeist präparierte Objekte aufwies. Es ist mehr als unwahrscheinlich, dass in der heutigen Ausstellung noch feuchte Objekte aus jener Zeit zu finden sind, denn die Präparier- und Konservierungsmethoden waren noch nicht so ausgereift wie heutzutage. Die knöchernen Belege, wie jener des Skeletts einer Frau, an der Fischer 1788 erstmals einen Kaiserschnitt durchführte, sind jedoch erhalten. 


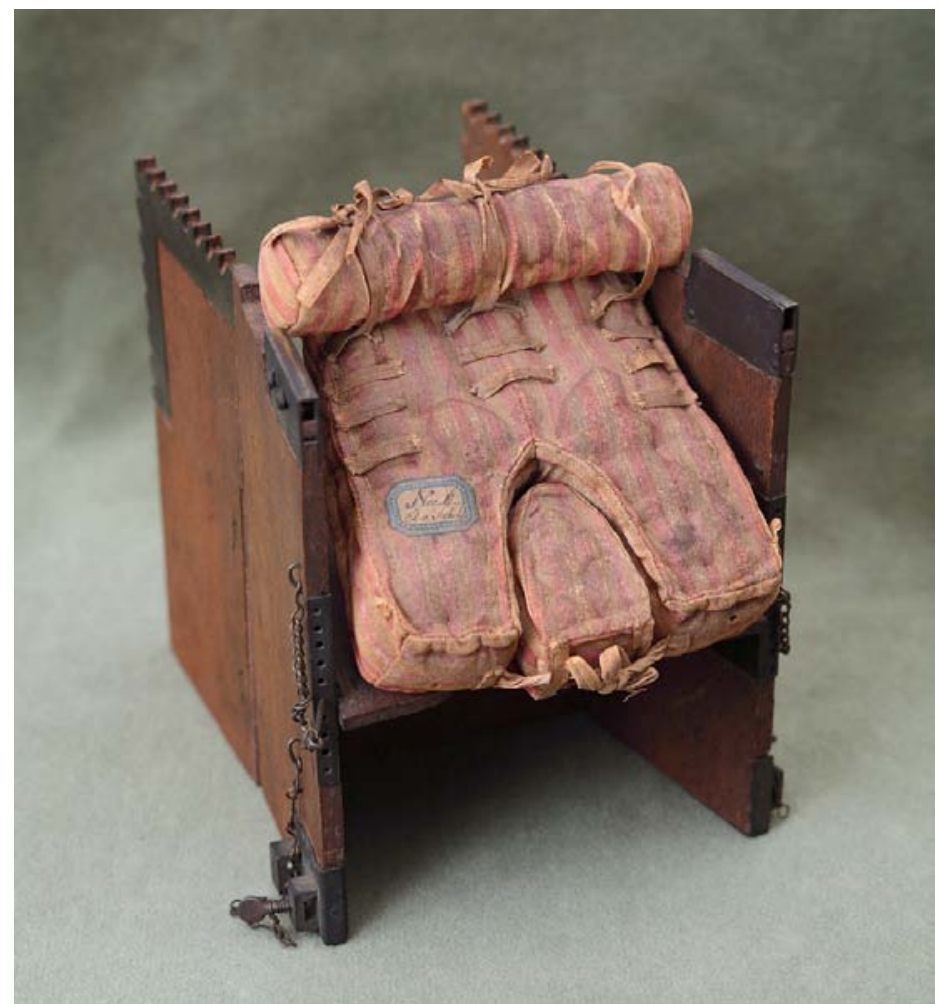

Abb. 36: Modell eines Gebärstuhls von Adam Elias von Siebold

Osiander erweiterte die Sammlung mit dem Ziel, den Studenten anschauliches Lehrmaterial zu liefern, auf 5000 Präparate, nannte sie „Museum Anatomicum Osiandrium " und katalogisierte sie, ohne jedoch eine Systematik zu erreichen, denn er kam über einen Zettelkasten nicht hinaus. Außerdem sammelte er Bücher, Dissertationen und geburtshilfliche Instrumente. Von den letztgenannten legte er 1804 einen systematischen Katalog an.

Das Spektrum des Bestandes reicht von Geburtszangen über Geburtsstühle bis zu anatomischen und chirurgischen Geräten, von denen einige bis heute in der Ausstellung zu besichtigen sind.

Auch dem Nachfolger Osianders Ludwig Caspar Julius Mende (1779-1832) gelang es, die Sammlung um rund 100 Objekte zu bereichern. Als 1833 Eduard Caspar Jacob von Siebold auf den Lehrstuhl berufen wurde, brachte er die umfangreiche, systematisch aufbereitete geburtshilfliche Sammlung seines Vaters mit nach Göttingen, die bei seinem Tod 1861 von der Witwe von Siebolds der Universität für 1700 Taler überlassen wurde. 


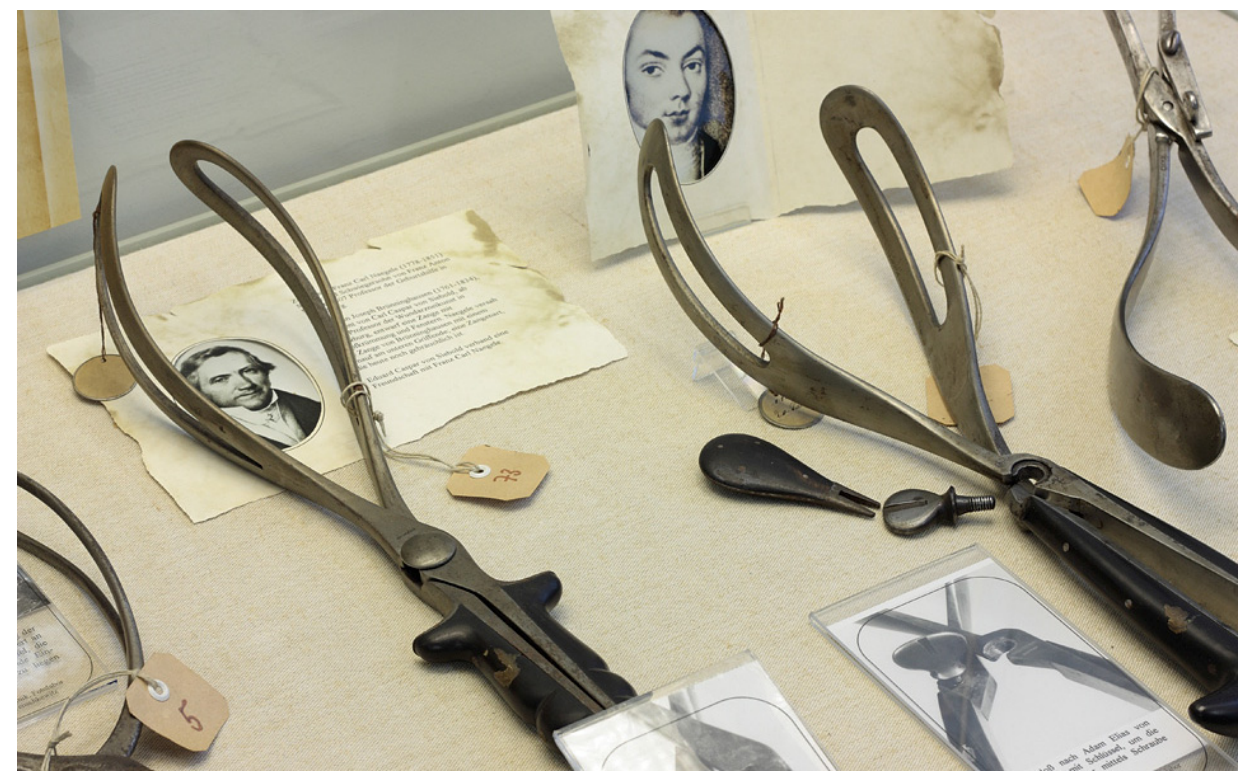

Abb. 37: Geburtszange nach Franz Karl Naegele (1778-1851) (links)

Geburtszange nach Adam Elias von Siebold (1775-1828) (rechts)

Eine Neuordnung vollzog sich dann unter Hermann Schwartz (1821-1890) und Jacob Henle. Die Sammlung wurde sachgerecht untergebracht, verdorbene und verrottete Präparate wurden entfernt, andere Objekte aus Platzgründen an andere Institute verkauft oder verschenkt. Im Wesentlichen war Schwartz ein Nachlassverwalter. In den folgenden Jahren erfuhr der Bestand keine nennenswerten Veränderungen. Nur neuere geburtshilfliche Instrumente und gynäkologische Medikamente wurden verzeichnet. Heinrich Martius bearbeitete dann 1935 und 1938 zwei Teilgebiete der Sammlung unter medizinhistorischen Gesichtspunkten. 1984 wurde eine systematische Erfassung und Katalogisierung der Bestände eingeleitet, ein Findbuch angelegt und ein Karteikartensystem aufgebaut, das u.a. den Verwendungszweck und die technischen Charakteristika der Objekte verzeichnet. 1995 entstand in den Räumen des Instituts für Ethik und Geschichte der Medizin eine Dauerausstellung, die regen Zuspruch erfährt. ${ }^{267}$

\section{Die „Blumenbachsche Schädelsammlung“ in der Göttinger Anatomie}

In der Göttinger Anatomie befindet sich die Blumenbachsche Schädelsammlung mit 850 Schädeln und Abgüssen. Johann Friedrich Blumenbach, ein aufgeklärter Universalgelehrter, betätigte sich in der Nachfolge Albrecht Hallers als Naturfor- 
scher und Professor der Medizin in Göttingen. Sein 1779 veröffentlichtes „Handbuch der Naturgeschichte" galt als richtungsweisend und erfuhr mehrere Auflagen, von denen besonders die 1799 erschienene sechste wichtig ist, da er dort über Knochenfunde aus der Gegend von Osterode/Harz berichtet, die das Mammut und das Wollhaarige Nashorn beschreiben.

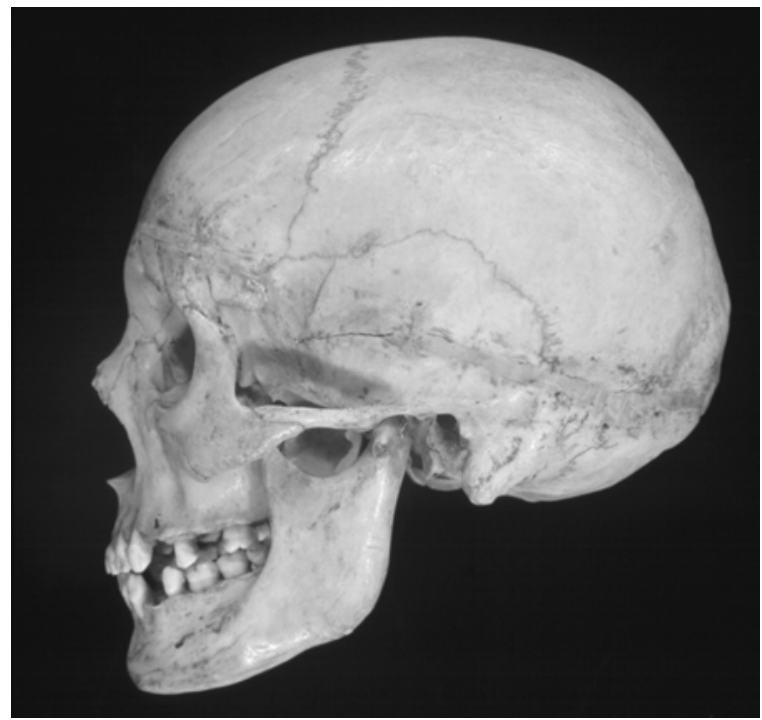

Abb. 38: Schädel einer Georgierin

In seiner Dissertation „De generis humani varietate nativa“ stellt er fünf morphologische Varietäten des Menschengeschlechts fest und beweist diese mit einer Schädelsammlung. Diese fünf Schädel sind immer noch ausgestellt: der Schädel einer Georgierin, eines Tungusen, eines Karaiben, einer Afrikanerin und eines Polynesiers. Vor allem dieser letztgenannte ist aufschlussreich, da an ihm eine perimortale Hiebverletzung durch eine scharfe Klinge zu erkennen ist. Blumenbach konnte seine Sammlung erweitern, da er immer wieder von Freunden, ausländischen Professoren und ehemaligen Studenten Schädel aber auch andere ethnologisch interessante Sammlungsstücke geschenkt bekam. Einige Schädel weisen handschriftliche Notizen Blumenbachs auf.

Paläopathologisch und medizinhistorisch aufschlussreich sind trepanierte Schädel, die aus der ursprünglichen Sammlung Blumenbachs stammen. Alle Schädel sind nach der gleichen Methode, die bereits Hippokrates beschrieb, trepaniert, nämlich intravital mit einem Kronentrepan. Der Schädel eines Russen lässt eine gut verheilte Operationsöffnung erkennen, während der Schädel eines Tartaren 
keine Heilungsspuren aufweist, sodass behauptet werden kann, dass der Patient an den Folgen der Trepanation verstarb.

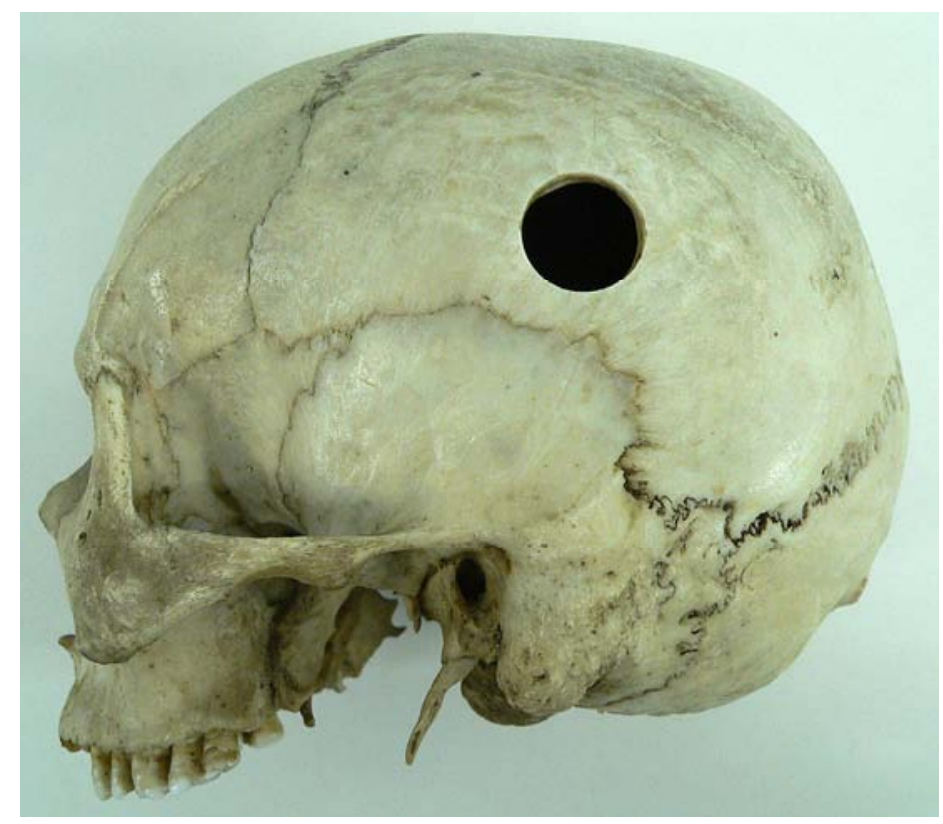

Abb. 39: Schädel eines Tartaren mit nicht verheilter Trepanationsöffnung

Kulturhistorisch und archäologisch bemerkenswert sind der Schädel eines Etruskers aus Tarquinia und ein Mumienkopf aus Ägypten, von dem Blumenbach noch die Binden teilweise abgetragen hat. Von seiner zweiten Italienreise kommend, schenkte Johann Wolfgang von Goethe Blumenbach den Abguss des Schädels von Raffael. Von großem Interesse sind auch zwei „Gallsche Schädel“, die an die medizinische Pseudowissenschaft, die Phrenologie, erinnern. Das Exponat zeigt die Lokalisation der von Gall beschriebenen „Hirnorgane“. Gall war der Überzeugung, dass sich die Organe der Hirnrinde durch Wölbungen an der Schädeloberfläche tasten ließen, wodurch er dann auf die Intelligenz eines Menschen schließen konnte. Die Nachfolger Blumenbachs konnten die Sammlung ebenfalls ergänzen.

1944 wurden die Schädel sorgsam verpackt und aus Angst vor drohenden Bombenangriffen im Café Waldschlösschen bei Bremke ausgelagert. Die Anatomie, die sich in der Nähe des Bahnhofs befand, wurde tatsächlich durch Bomben zerstört, wodurch auch der umfangreiche Katalog, der auch wichtige anthropologische Untersuchungen enthielt, verbrannte. Aber die Auslagerung bewahrte die Sammlung vor noch größerem Schaden. Diese bemerkenswerte Sammlung genießt ebenfalls internationales Ansehen. ${ }^{268}$ 



\section{Ehrungen}

Neben der Ehrenpromotion verleiht die Medizinische Fakultät im Gedenken an ihre beiden bedeutendsten Mediziner die "Albrecht von Haller-Medaille" und die "Jacob Henle-Medaille" in einer akademischen Feier.

\section{Die Albrecht von Haller-Medaille}

Die "Albrecht von Haller-Medaille", deren Verleihung der Fakultätsrat auf seiner Sitzung am 17.11.1958 beschlossen hatte, wird Göttinger oder auswärtigen Persönlichkeiten verliehen, die sich besonders hohe Verdienste von nachhaltiger Wirkung und Dauer im Zusammenhang mit den wissenschaftlichen und/oder ärztlichen Aktivitäten des Fachbereichs Medizin der Georg-August-Universität Göttingen erworben haben.

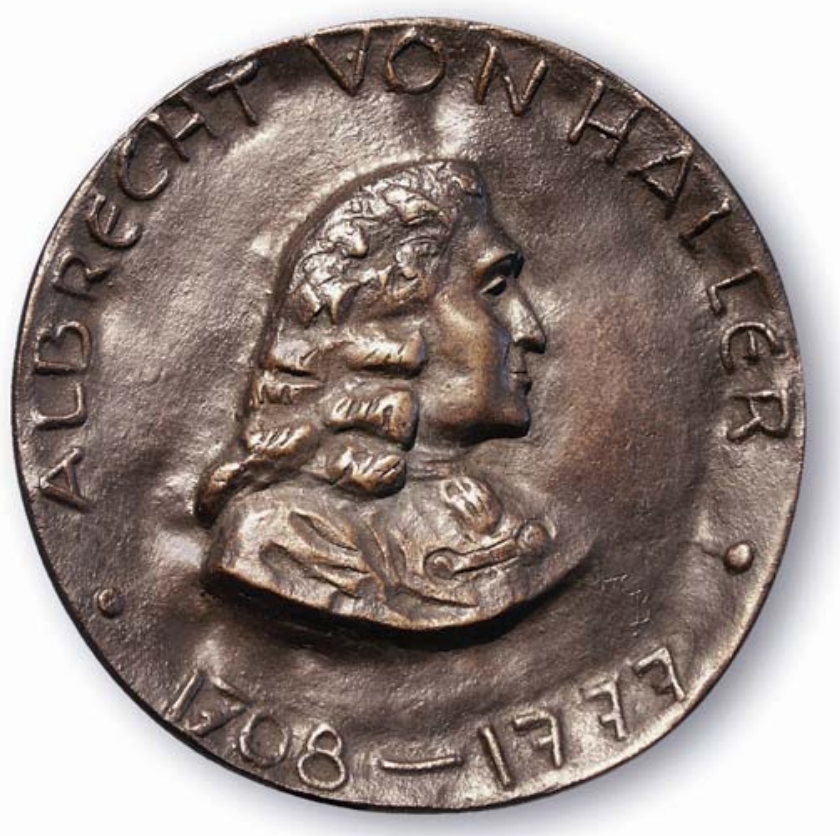

Abb. 40: Die Albrecht von Haller-Medaille 


\section{Preisträger der Albrecht von Haller-Medaille}

Name und Ort

Dr. h.c.

Bruno Hauff,

Stuttgart

Prof. Dr. Dr. h.c.

Heinrich Martius,

Göttingen

Prof. Dr.

Rudolf Stich,

Göttingen

Prof. Dr.

Hans Meyer,

Marburg

Dr.

Ferdinand Springer,

Heidelberg

Prof. Dr. Dr. h.c.

Hans Kleinschmidt,

Berlin

Prof. Dr. Dr. h.c. Dr. h.c.

Karl Thomas,

Göttingen

Prof. Dr. Dr. h.c.

Benno Gruber,

Göttingen

Prof. Dr. Dr. h.c.

Albrecht Peiper,

Leipzig

Prof. Dr. Dr. h.c.

Rudolf Schoen,

Göttingen

Prof. Dr. Dr.

Wilhelm Meyer,

Göttingen

Prof. Dr.

R. C. Mac Keith,

London
Anlass

Datum

75. Geburtstag

16.02.1959

75. Geburtstag

02.01 .1960

85. Geburtstag

19.07.1960

Ehrenpromotion der

21.06.1961

Phil. Fakultät Kiel

80. Geburtstag

29.08.1961

225 Jahrfeier der Georg-August-

14.11.1962

Universität Göttingen

80. Geburtstag

04.12.1963

80. Geburtstag

22.02.1964

Tagung Nordw.-dt. Gesellschaft für Kin-

07.07.1964 derheilkunde in Göttingen

80. Geburtstag

31.01 .1972

80. Geburtstag

02.04.1976

Congress der European Federation of

24.05.1977

Child Neurology Societies in Braunlage 
Prof. Dr.

Gerhard Joppich, Göttingen

Prof. Dr.

Heinz Kirchhoff,

Göttingen

Prof. Dr.

Gustav Born,

Cambridge

Prof. Dr.

Josef Koncz,

Göttingen

Prof. Dr.

Steffen Berg,

Göttingen

Prof. Dr. Dr. h.c.

Hans-Jürgen Bretschneider, Göttingen

Prof. Dr. Dr. h.c.

Werner Creutzfeldt,

Göttingen

Prof. Dr.

Gerhard Schmidt,

Göttingen

Prof. Dr. Dr. h.c.

Erwin Deutsch,

Göttingen
Feierlichkeiten der Universität aus Anlass

14.12.1977

des 200. Todestages von Albrecht von

Haller

Feierlichkeiten der Universität aus Anlass

des 200. Todestages von Albrecht von

Haller

große Verdienste um die Erforschung von

Thrombocytenfunktionen für den Blut-

kreislauf und die Blutgerinnung

für seine Arbeit auf allen Gebieten der

THG-Chirurgie und seine Verdienste um

die Entwicklung des Faches in Deutsch-

land etc.

wiss. Arbeiten auf dem Gebiet der

Rechtsmedizin und dessen Entwicklung,

Studien auf dem Gebiet der Paleopatho-

logie etc.

wiss. Arbeiten auf dem Gebiet der Physio-

logie des Herz-Kreislaufsystems, beson-

ders der Energetik der Herzfunktion,

Methoden zur Protektion und Konservie-

rung von Organen in der offenen Trans-

plantationschirurgie etc.

wiss. Arbeiten auf dem Gebiet der Inne-

ren Medizin, insbesondere der Physiologie und Pathophysiologie etc.

in dankbarer Würdigung seiner großen

Verdienste und seines vorbildlichen Wir-

kens als Lehrer und Studiendekan und

Promotor

in Würdigung seiner Verdienste um die Erforschung und prakt. Anwendung des Medizinrechts und Engagements in der Ethik-Kommission. Tatkräftige Verbindung zwischen Juristischer und Medizinischer Fakultät
12.01.1979

27.09.1991

30.06 .2000

14.12.1977

05.02.1988

30.04 .1993

10.02.1995

20.06.2002 


\section{Die Jacob Henle-Medaille}

Auf seiner Sitzung vom 26.5.1986 beschloss der Fachbereichsrat, die Jacob HenleMedaille für eine herausragende, für die Medizin relevante wissenschaftliche Leistung zu verleihen. Mit der Verleihung verbunden ist die Jacob Henle-Vorlesung.

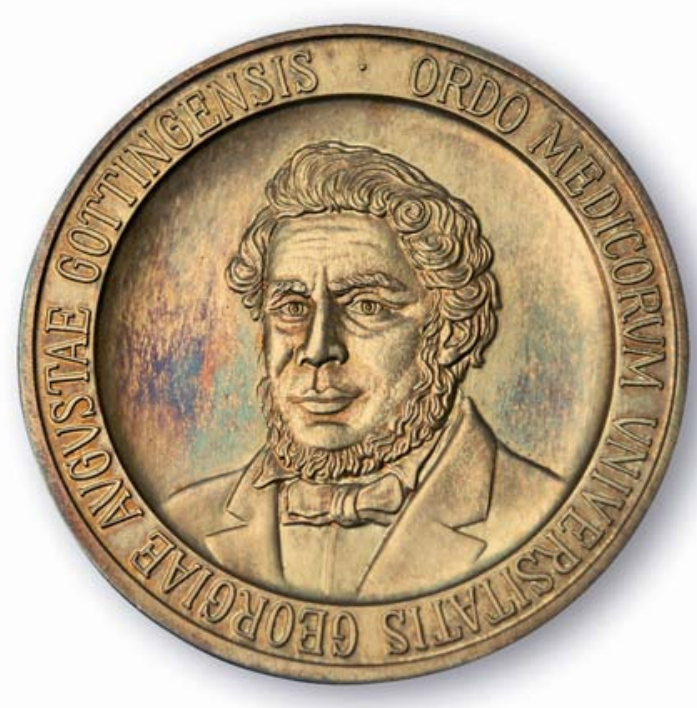

Abb. 41: Die Jacob Henle-Medaille

\section{Trägerinnen und Träger der Jacob Henle-Medaille}

\section{Name und Ort}

Prof. Dr. Dr. h.c. Karl-Julius Ullrich, Frankfurt

Prof. Dr. Dr. h.c. mult. Paul Janssen, Beerese, Belgien

Prof. Dr. Wilhelm Kritz, Heidelberg

Prof. Dr. Wolfgang Gerok, Freiburg

Prof. Dr. Bruno Lunenfeld, Tel Aviv, Israel

Prof. Dr. Hans Peter Hofschneider, Martinsried

Prof. Dr. Dr. h.c. Friedrich Vogel, Heidelberg

Prof. Dr. Dr. h.c. Andreas Oksche, Gießen

\section{Datum}

30.11 .1988

25.01.1990

14.12.1990

10.12.1991

22.01.1993

12.11.1993

20.01.1995

19.01.1996 
Name und Ort

Prof. Dr. Harald zu Hausen, Heidelberg

Prof. Dr. Bengt Hagberg, Göteborg, Schweden

Prof. Dr. Rainer Greger, Freiburg

Prof. Dr. Friedmund Neumann, Berlin

Prof. Dr. Dr. h.c. mult. Hans-Peter Zenner, Tübingen

Prof. Dr. Helga Rehder, Marburg

Prof. Dr. Hans-Jochen Kolb, München

Prof. Dr. Dr. h.c. Klaus Thurau, München
Datum

24.01.1997

23.01.1998

23.11.1998

02.02.2001

12.04.2002

16.05.2003

14.05.2004

30.01 .2009 



\section{Chronologie}

1734 Gründung der Georg-August-Universität Göttingen

Erste Vorlesung durch den Philosophen

Samuel Christian Hollmann (1696-1787)

Johann Wilhelm Albrecht (1703-1736) erster Professor

an der Medizinischen Fakultät

Eröffnung der Anatomie im Turm des Albanitors

1735 Erster Unterricht an der Medizinischen Fakultät

1736 Amtsantritt von Georg Gottlob Richter (1694-1773) und Albrecht Haller (1708-1777)

1737 Eröffnung des Anatomischen Theaters am botanischen Garten Inauguration der Georg-August-Universität Göttingen

1739 Anlegung des botanischen Gartens

1751 Armenhospital St. Crucis als Gebärklinik

Amtsantritt von Johann Georg Roederer (1726-1763)

1752 Beginn des Lehrbetriebs in der Geburtshilfe

1753 Weggang Albrecht Hallers

1755 Erster praktisch-klinischer Unterricht durch Johann Gottfried Brendel (1712-1758)

1773 Eröffnung des Collegium clinicum unter Ernst Gottfried Baldinger (1738-1804)

1778 Amtsantritt von Johann Friedrich Blumenbach (1752-1840)

1781 Eröffnung des akademischen Hospitals unter August Gottlieb Richter (1742-1812)

1791 Eröffnung des Accouchier-Hospitals

1792 Amtsantritt von Friedrich Benjamin Osiander (1792-1882)

1809 Eröffnung von Himlys Hospital am Leinekanal

1821 Beginn der Zahnheilkunde durch Johann Heinrich Pauli (1795-1850)

1829 Eröffnung des klassizistischen Neubaus der Anatomie

1840 Institutionalisierung der Physiologie unter Rudolf Wagner (1805-1864)

1850 Eröffnung des Ernst-August-Hospitals

1852 Institutionalisierung der Pathologie unter August Foerster (1822-1865)

Amtsantritt von Jacob Henle (1809-1885)

1862 Entdeckung und Veröffentlichung der „Henleschen Schleife“ 
1866 Eröffnung der provinzialständigen Landes-Irrenanstalt unter Ludwig Meyer (1826-1900)

1868 Institutionalisierung der Augenheilkunde unter Carl Schweigger (1830-1905)

1873 Institutionalisierung der Pharmakologie unter Wilhelm Marmé (1832-1897)

1877 Beginn der Hals-, Nasen- und Ohrenheilkunde unter Kurd Bürkner (1853-1913)

1878 Eröffnung der Königlichen Universitäts-Poliklinik für

Ohren- und Nasenkrankheiten

1881 Beginn der Physiologischen Chemie und Hygiene unter Karl Flügge (1847-1923)

1883 Institutionalisierung der Medizinischen Chemie und Hygiene

1886 Eröffnung des Physiologischen Instituts

1889 Eröffnung der Chirurgischen Klinik

1891 Eröffnung der Medizinischen Klinik

Eröffnung des Pathologischen Instituts

1894 Wiederbeginn der Zahnheilkunde unter Carl Heitmüller (1864-1951)

1896 Eröffnung der Frauenklinik

1903 Beginn der Gerichtsmedizin unter Paul Stolper (1865-1906)

1906 Eröffnung der Augenklinik

1907 Beginn der Kinderheilkunde unter Bruno Salge (1872-1924)

1911 Eröffnung der Kinderklinik

1917 Anfänge des Faches Haut- und Geschlechtskrankheiten unter Erhard Riecke (1869-1939)

1919 Neugründung des Zahnärztlichen Instituts

1928 Eröffnung der Hautklinik am Steinsgraben

1938 Neubau des Physiologischen und Physiologisch-Chemischen Instituts

1941 Einrichtung des Hygienischen Instituts

1952 Erster therapeutischer Einsatz der Elektronenschleuder

1955 Eröffnung der Neurologischen und Psychiatrischen Klinik

1957 Eröffnung der Zahnärztlichen Klinik und Poliklinik

1959 Eröffnung der Hautklinik am Kreuzbergring

1962 Eröffnung der Anatomie am Kreuzbergring

1977 Beginn des Umzugs der einzelnen Kliniken in das Universitätsklinikum

1988 Abschluss des Umzugs

1999 Installierung des Vorstandes nach dem Integrationsmodell Niedersachsens

2003 Georg-August-Universität in der Trägerschaft einer Stiftung öffentlichen Rechts mit der Teilstiftung Universitätsmedizin 


\section{Zentren und Abteilungen der Medizinischen Fakultät im Jahre 2009}

Zentrum Anaesthesiologie-, Rettungs- und Intensivmedizin

Abt. Anaesthesiologie I

Abt. Anaesthesiologie II -

Operative Intensivmedizin

Abt. Palliativmedizin

Zentrum Arbeits-, Sozial-, Umweltmedizin und Dermatologie

Abt. Allgemeine Hygiene und Umweltmedizin

Abt. Arbeits- und Sozialmedizin

Abt. Dermatologie, Venerologie und Allergologie

Zentrum Biochemie und Molekulare Zellbiologie

Abt. Biochemie I

Abt. Biochemie II

Abt. Entwicklungsbiochemie

\section{Zentrum Frauenheilkunde}

Abt. Gynäkologie und Geburtshilfe

\section{Zentrum Informatik, Statistik und} Epidemiologie

Abt. Bioinformatik

Abt. Genetische Epidemiologie

Abt. Medizinische Informatik

Abt. Medizinische Statistik

\section{Zentrum}

Anatomie

Abt. Anatomie und Embryologie

Abt. Anatomie und Zellbiologie

Abt. Neuroanatomie

Zentrum Augenheilkunde und Hals-, Nasen-, Ohrenheilkunde

Abt. Augenheilkunde

Abt. Hals-Nasen-Ohrenheilkunde

\section{Zentrum}

Chirurgie

Abt. Allgemein- und Viszeralchirurgie

Abt. Orthopädie

Abt. Unfallchirurgie, Plastische- und

Wiederherstellungschirurgie

Abt. Urologie

Abt. Thorax-, Herz- und Gefäßchirurgie

Zentrum Hygiene und Humangenetik

Abt. Humangenetik

Abt. Medizinische Mikrobiologie

Abt. Transfusionsmedizin

Abt. Virologie

Abt. Zelluläre und Molekulare Immunologie

\section{Zentrum}

\section{Innere Medizin}

Abt. Allgemeinmedizin

Abt. Gastroenterologie und Endokrinologie

Abt. Hämatologie und Onkologie

Abt. Nephrologie und Rheumatologie

Abt. Kardiologie und Pneumologie

Abt. Klinische Chemie 
Zentrum Kinderheilkunde und Jugendmedizin

Abt. Pädiatrie I mit Schwerpunkt

Hämatologie und Onkologie

Abt. Pädiatrie II mit Schwerpunkt

Neuropädiatrie

Abt. Pädiatrie III mit Schwerpunkt Pädiatrische

Kardiologie und Intensivmedizin

\section{Zentrum Pathologie und Rechtsmedizin}

Abt. Gastroenteropathologie

Abt. Neuropathologie

Abt. Pathologie

Abt. Rechtsmedizin

\section{Zentrum Physiologie und}

Pathophysiologie

Abt. Herz- und Kreislaufphysiologie

Abt. Neuro- und Sinnesphysiologie

Abt. Neurophysiologie und Zelluläre Biophysik

Abt. Vegetative Physiologie und

Pathophysiologie

\section{Zentrum}

\section{Radiologie}

Abt. Diagnostische Radiologie

Abt. Neuroradiologie

Abt. Nuklearmedizin

Abt. Strahlentherapie und Radioonkologie

\section{Interdisziplinäre}

\section{Abteilungen}

Abt. Molekulare Onkologie (GZMB)

Abt. Neuroimmunologie

Abt. Stammzellbiologie (CMPB)

\section{Zentrum}

Neurologische Medizin

Abt. Klinische Neurophysiologie

Abt. Neurochirurgie

Abt. Neurologie

\section{Zentrum Pharmakologie und Toxikologie}

Abt. Klinische Pharmakologie

Abt. Pharmakologie

\section{Zentrum}

\section{Psychosoziale Medizin}

Abt. Ethik und Geschichte der Medizin

Abt. Kinder- und Jugendpsychiatrie und Psychotherapie

Abt. Medizinische Psychologie und Medizinische Soziologie

Abt. Psychiatrie und Psychotherapie

Abt. Psychosomatische Medizin und Psychotherapie

\section{Zentrum Zahn-, Mund-} und Kieferheilkunde

Abt. Kieferorthopädie

Abt. Mund-, Kiefer- und Gesichtschirurgie

Abt. Prothetik

Abt. Präventive Zahnmedizin, Parodontologie und Kariologie 


\section{Anmerkungen}

Die Angaben beziehen sich auf Kapitel 16. Quellen und Literatur

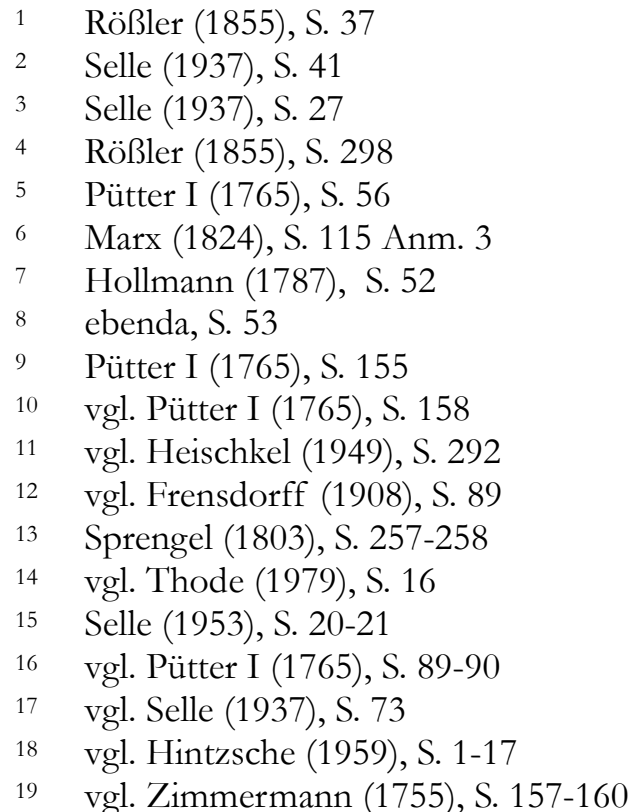

Bei dieser ersten von seinem Schüler verfassten Biographie, die Haller in hohem Maße glorifiziert, die literarischen und wissenschaftlichen Gegner dagegen scharf kritisiert, und auf die in der Folgezeit ständig zurückgegriffen wird, gilt es jedoch zu bedenken, dass Haller durch Hinweise und Vorgaben das gesamte Manuskript beeinflusst und vor der Drucklegung gelesen sowie korrigiert hat. Vgl. Hintzsche (1959), S.3

Unverständlich bleibt dabei allerdings, warum Haller sich in seiner im gleichen Jahr erschienenen Rezension von der Lebensdarstellung distanzierte. Vielleicht war die negative öffentliche Reaktion Ursache für diesen Umschwung. Vgl. Haller (1755), S. 615-616 vgl. Rößler (1855), S. 354 
21

ebenda, S. 35. Es gilt als wahrscheinlich, dass der Bau der Kirche der reformierten Gemeinde in unmittelbarer Nähe von Hallers Wohnhaus auf seine Initiative zurückging.

Zimmermann (1755), S. 162-163

vgl. Pütter I (1765), S. 233

vgl. Eulenburg (1904), S. 148

vgl. Ebel (1961), S. 156 (Kap. I, \8)

vgl. ebenda, S. 35

vgl. Pütter I (1765), S. 234

vgl. Zimmermann (1755), S. 163

vgl. Haller I (1757), S. IX

"Non alia arte certe posset ad perfectionem anatomes propius accedi, si in Academia opportunitatibus divite hoc consilium per annos, \& per secula urgeretur." Haller I (1757), S. IX

vgl. Hintzsche (1959), S.7

vgl. Zimmermann (1755), S. 162, 165

vgl. Univ. Archiv, Med. Fak. 4, IV b, 6; (20. August 1738 und 26. November 1739)

vgl. Ebel (1961), S. 154

vgl. Univ. Archiv, Med. Fak. 4, IV a, 5; (27. Dezember 1751)

vgl. ebenda, (27. Dezember 1751)

vgl. ebenda, (27. Dezember 1751)

vgl. ebenda, (26. Dezember 1751)

vgl. Univ. Archiv, Med. Fak. 4, IV b, 4; (6. Mai 1752 Haller); (8. Mai 1752 Brendel)

vgl. ebenda, (17. Januar; 1. Februar; 4. März; 17. März 1753)

„Elementa Physiologiae Corporis Humani“ umfasst acht Bände, die zwischen 1757 und 1766 nach seiner Göttinger Zeit erschienen sind. Signifikant ist das Vorwort des ersten Bandes, in dem Haller die Grundzüge seines Selbstverständnisses umreißt.

vgl. Haller (1753), S. 114-158

vgl. Univ. Archiv, Med. Fak. 4, IV b, 8; (18. Oktober 1753)

vgl. Pütter I (1765), S. 159-161

vgl. Univ. Archiv, Med. Fak. 4, IV b, 15; (2. Mai 1764)

vgl. Eulner (1970), S. 35

vgl. Univ. Archiv, Med. Fak. 4, IV b, 15; (10. Oktober 1774; 26. November 1800; 17. Mai 1801; 22. Mai 1801)

Selle (1937), S. 223

vgl. Blumenbach (1781)

vgl. McLaughlin (1982), S. 357-372 
51

Kant ging im zweiten Teil seiner „Kritik der Urteilskraft“ auf Blumenbach ein. Vgl. Kant VIII (1968), S. 545 (\$ 81) und Goethe in seinem Aufsatz „Bildungstrieb“. Vgl. Goethe II, 7 (1892), S. $71-73$

vgl. Univ. Archiv, Med. Fak. 4, IV d, 15; (W.S. 1804/05)

vgl. Univ. Archiv, Med. Fak. 4, IV b, 20; (9. Mai 1819)

vgl. ebenda, (9. Mai 1826)

Richter (1936), S. 109

vgl. Langenbeck (1829), S. 5-9

vgl. Eulner (1970), S. 52

vgl. Eulner, Hoepke (1979), S. 14

Die drei anderen Aufsätze „Über Nervensympathien“, „Über Verlauf und Periodität der Krankheit" und „Über das Fieber" waren von gleicher Tragweite. Vgl. Henle (1840)

vgl. Henle (1841)

vgl. Merkel (1891), S. 203-206, 208

vgl. Henle (1846-1853)

vgl. Merkel (1891), S. 295

vgl. Henle (1862), S. 247-248

vgl. Henle (1872-1879), S. V

Merkel (1891), S. 362

vgl. Krumsteller (1958), S. 15

Baldinger (1789), S. 98

Frensdorff (1908), S. 90

vgl. Krumsteller (1958), S. 15-16

vgl. Pütter I (1765), S. 293

Frensdorff (1908), S. 93

vgl. Krumsteller (1958), S. 17-18

vgl. Ebstein (1889), S.72

vgl. Krumsteller (1958), S. 20-21

Baldinger (1789), S. 100

vgl. Krumsteller (1958), S. 30

vgl. ebenda, S. 35

vgl. ebenda, S. 40-41

vgl. Univ. Archiv, Kur. 4, IV b, 14; (29. Oktober 1778)

vgl. Univ. Archiv, Sekr. VI D, 475; (29. August 1778)

vgl. Univ. Archiv, Kur. 4, IV e, 14; (11. Januar 1781)

vgl. Pütter II (1788), S. 264

Winkelmann (1981), S. 48

vgl. Univ. Archiv, Sekr. VIII, 526/3; (19. Dezember 1780)

vgl. ebenda, (8. November 1781) 
87

88

vgl. Univ. Archiv, Kur. 4, IV e, 21; (3. März 1802)

Richter (1781), S. 376

vgl. Pütter II (1788), S. 265

vgl. Univ. Archiv, Kur. 4, IV b, 14; (17. September 1801; 13. April 1802)

vgl. ebenda, (29. Mai 1796)

Das Armenkrankenhaus, eine Einrichtung der Armenfürsorge, diente der ärztlichen Versorgung armer Kranker. Für die stationäre Behandlung gab es 1789 ein Krankenzimmer, die „Armenstube“ am Groner Tor. 1794 wurde das Armenkrankenhaus am Albanitor mit sechs Betten eröffnet.

vgl. Univ. Archiv, Kur. 4, IV e, 14; (11. Mai 1797)

vgl. Zimmermann (2005), S. 1250

Richter (1793), S. IX

ebenda, S. 60

vgl. Zimmermann (2005), S. 596

vgl. Univ. Archiv, Kur. 4, IV e, 2; (30. September 1803)

vgl. ebenda, (3. Mai 1807; 4. Mai 1807)

vgl. Univ. Archiv, Kur. 4, IV f, 3; (18. Mai 1807; 6. August 1807)

vgl. Winkelmann (1981), S. 212-213

vgl. Himly (1810), S. 529-531

vgl. ebenda, S. 532-542

vgl. Univ. Archiv, Kur. 4, IV e, 40; (15. November 1835)

vgl. ebenda, (28. November 1835)

vgl. Univ. Archiv, Kur. 4, IV e, 2; (23. März 1837)

vgl. Haselhorst (1964), S. 25-28

vgl. Univ. Archiv, Kur. 4, VI D, 479; (6. August 1851)

vgl. Univ. Archiv, Kur. 13, c, 1; (30. April 1846)

vgl. Univ. Archiv, Sekr., VI D, 479; (6. August 1851)

vgl. Univ. Archiv, Kur. 13, c, 1; (19. März 1846)

vgl. Univ. Archiv, Kur. 4, IV e, 65; (10. März 1854; 12. März 1854)

vgl. Univ. Archiv, Kur. 13, c, 58; (12. April 1876; 16. April 1876)

vgl. Univ. Archiv, Kur. 4, IV e, 71; (2. Juni 1861; 6. September 1861)

vgl. Univ. Archiv, Kur. 4, IV e, 73; (14. Februar 1867)

vgl. Univ. Archiv, Kur. 4, IV e, 78a; (12. Dezember 1874; 25. Juni 1875)

vgl. Univ. Archiv, Kur. 4, IV e, 79a; (7. August 1874)

vgl. Univ. Archiv, Kur. 4, IV e, 76; (18. Februar 1874)

vgl. ebenda, (14.Februar 1879); Mantius (1937), S. 18

vgl. Univ. Archiv, Kur. 13 c, 10; (19. Mai 1877; 12. Juli 1879)

vgl. Ebstein (1907), S. 367-378; ders. (1912), S. IV-X

vgl. König (1891), S. 246-271

vgl. Ebstein (1891), S. 235-246 
vgl. Zimmermann (1755), S. 272

Osiander I (1794), S. XLII

vgl. Martius (1931), S. 14

vgl. Osiander I (1794), S. XLIV-XLV

vgl. ebenda, S. XLVI

vgl. ebenda, S. XLVIII

Pütter II (1788), S. 259

vgl. Osiander I (1794), S. LVI-LVII

„Ferner ist man jetzt willens einen Akkouchier-Palast zu bauen, aber die ExperimentalPhysik, die noch die Basis von so vielen dem Staat nützlichen Kenntnissen ist, die eine der edelsten Beschäftigungen des Geistes für alle Stände gewährt, wird so ganz. vergessen. " Lichtenberg IV (1967), S. 664

Osiander, der 1792 als erster Leiter die Dienstwohnung im dritten Geschoss des Neubaus bezog, verteidigte hingegen das Hospital: „Der Reiche thut freylich wohl, wenn er Palläste nur von der Dauer eines Menschenalters bauet. Seine Nachkommen finden seinen Bangeschmack doch veraltert, errichten neue Palläste, und überlassen das geerbte Denkmal des Stolzes und der Verschwendung gewöhnlich den Unken und Eulen. Aber Hospitäler, die Denkmäler der Menschenliebe und Woblthätigkeit, müssen für viele Jahrhunderte, für viele Generationen gebaut werden, denn auch den Nachkommen sind sie noch ehrwürdig und ibr Nutzen und Rubm wächst mit den Jahren. "Osiander I (1794), S. LI

Goethe, der 1801 Göttingen besuchte, fand das Accouchier-Hospital „sonderbar erbaut" und meinte damit wohl in erster Linie das Treppenhaus.

„Und wie denn jeder Ort den fremden Ankömmling zerstreuend bin=und herzieht und unsere Fähigkeit, das Interesse mit den Gegenständen schnell zu wechseln, von Augenblick zu Augenblick in Anspruch nimmt, so wußte ich die Bemühung des Professors Osiander zu schätzen, der mir die wichtige Anstalt des neu=und sonderbar erbauten Accouchirhauses, so wie die Behandlung des Geschäftes erklärend zeigte." Goethe I, 35 (1892), S.98

Osiander hat die Bedingungen, unter denen dies „heimlichste Wochenbett“ stattfinden konnte, detailliert festgelegt. Der Kontakt musste schriftlich über einen Dritten und die Bezahlung der Kosten über eine Vertrauensperson oder über ein Handelshaus erfolgen. Der Aufenthalt in der Entbindungsanstalt vollzog sich in absoluter Anonymität. Für den heimlichen Eintritt in das Accouchierhaus war im nördlichen Teil eine besondere Treppe vorhanden, die direkt in die dritte Etage führte. Die Entbindung übernahm Osiander selbst. Ohne Nachfrage über die Herkunft wurde das Kind im Hospital getauft, eine Wöchnerin als Amme gefunden und gegen Sicherstellung des Pflegegelds eine gute Pflegemutter auf dem Lande vermittelt. 
"Jedermann weiss, wie sehr die Rube und Woblfahrt einer ganzen Familie zunveilen durch eine einzige schwache Stunde einer Person aus ibrer Mitte gestört und auf Jabre lang zerrïttet wird, und zu welch traurigen Schritten die Furcht vor öffentlicher Beschämung verleitet, und niemand wird daher das Woblthätige einer Anstalt misskennen, durch welche Zufriedenheit, Ehre und Glück eines Einzelnen, und einer ganzen Familie erbalten wird. " Osiander I (1794), S. LXXXV

vgl. Osiander I (1794), S. LXV-LXXIX

vgl. Pütter III (1820), S. 308-313

vgl. Winkelmann (1983), S. 308-311

vgl. Siebold (1839-1845), S. 604

vgl. Deinhard (1986), S. 48-52

vgl. Küntzel (2003)

vgl. Eulner (1970), S. 289, 291, 294

vgl. Martius (1931), S. 40-44

vgl. Hagene (2006), S. 62-64

vgl. ebenda, S. 26

vgl. Hippel (1907), S. 217-226

vgl. Eulner (1970), S. 206

vgl. ebenda, S. 219

vgl. Radke (1974), S. 6-29

Henoch (1890), S. 130

vgl. Radke (1974), S. 55

vgl. ebenda, S. 69, 96, 101, 225-230

vgl. Univ. Archiv, Kur. 4, IV e, 113; (17. Dezember 1906). Damit wollten die Eltern ihrem einzigen und früh verstorbenen Sohn Carl Dreger (1879-1903), der unter Wilhelm Ebstein Assistent an der medizinischen Klinik war, ein Denkmal setzen. Noch heute erinnert eine Gedenktafel im Eingangsbereich des Hauses daran.

Uriv Archiv, Kur. 4, IV e, 115; (15. Januar 1911)

vgl. Chronik (1911), S. 43

vgl. Univ. Archiv, Kur. 13 c, 18; (12. Januar 1918)

vgl. ebenda, (23. April 1923)

vgl. Radke (1974), S. 91-92

vgl. ebenda, S. 119

vgl. ebenda, S. 125

Eulner (1970), S. 256

vgl. Welter (1935), S. 40-59

vgl. Ebstein (1891), S. 239

vgl. Welter (1935), S. 63-66

vgl. Geier (1987), S. 21-24 


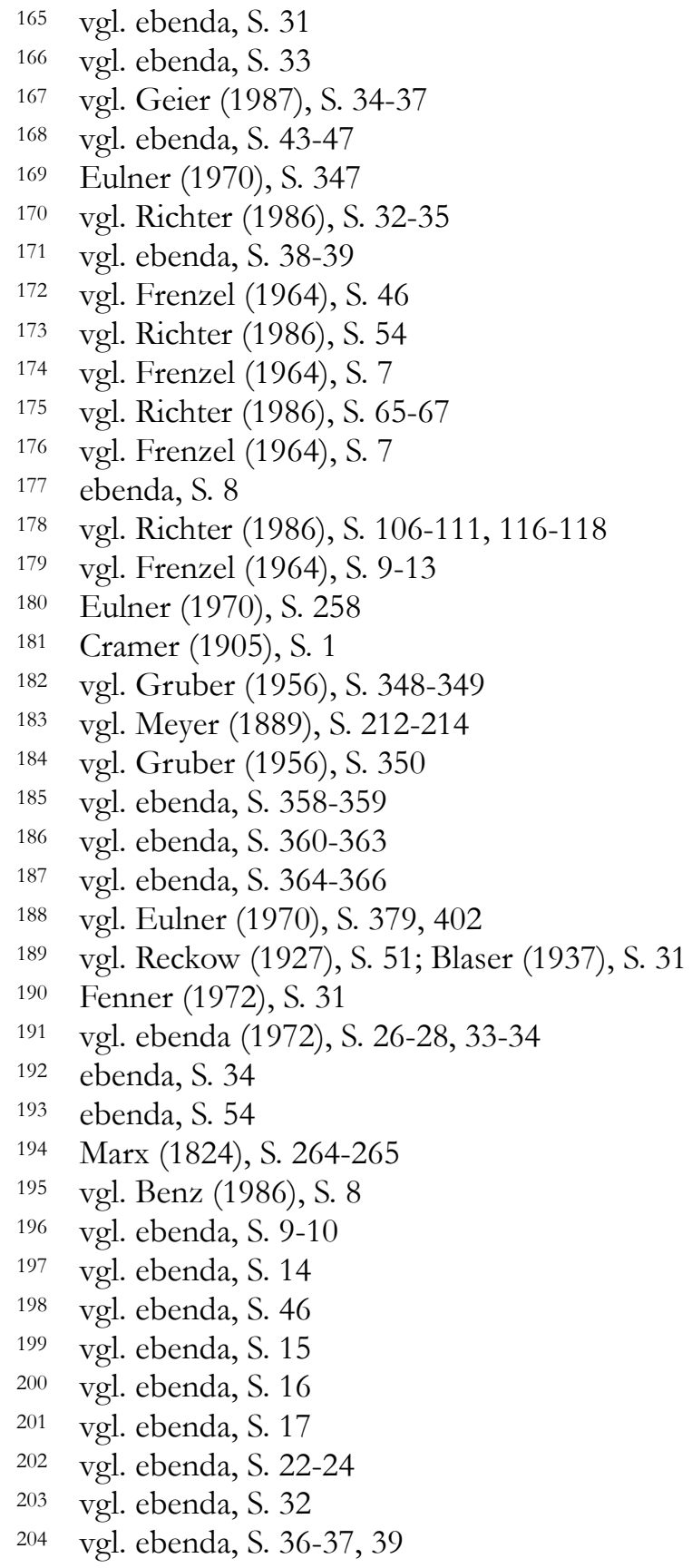


205

206

207

208

209

210

211

212

213

214

215

216

217

218

219

220

221

222

223

224

225

226

227

228

229

230

231

232

233

234

235

236

237

238

239

240

241

242

243

vgl. ebenda, S. 45

vgl. ebenda, S. 47-49

vgl. ebenda, S. 51-57

vgl. ebenda, S. 59, 64, 67, 74

vgl. ebenda, S. 78

vgl. ebenda, S. 72-73

vgl. ebenda, S. 89

vgl. ebenda, S. 78-81

vgl. ebenda, S. 100-101

vgl. ebenda, S. 103

vgl. ebenda, S. 106-107

vgl. ebenda, S. 112-114

vgl. ebenda, S. 116

vgl. ebenda, S. 117-118

vgl. Eulner (1970), S. 97

vgl. Kohlhagen (1935), S. 5

ebenda, S. 14

vgl. ebenda, S. 16-17

vgl. Orth (1891), S. 271-277

vgl. Eulner (1970), S. 67

vgl. ebenda, S. 94

vgl. ebenda, S. 81-82, 608

vgl. Rieberer (1990), S. 9-12

vgl. ebenda, S.12

vgl. ebenda, S. 28-29

vgl. Univ. Archiv, Kur. Dek. Med. 54

vgl. Eulner (1970), S. 81

vgl. Univ. Archiv, Kur. 16, III, Ce 3; (9. September 1941; 2. Oktober 1941)

vgl. Eulner (1970), S. 119

Lauer (1966), S. 31

vgl. Luhn (1971), S. 23

vgl. ebenda, S. 22

vgl. Univ. Archiv, Med. 66

vgl. Luhn (1971), S. 22

vgl. Univ. Archiv, Acta XA, 555c, 1

vgl. Hasselhorn/Weinreis (1983), S. 57

vgl. Georg-August-Universität zu Göttingen. Amtliches Namensverzeichnis.

Winterhalbjahr 1932/33, S. 8-11

vgl. Göttinger Tageblatt vom 26. Juli 1933, S. 3

vgl. Univ. Archiv, Med. 5 
244 vgl. Univ. Archiv, Kur. 16. III, Ad. 53

245 vgl. Univ. Archiv, Med. 95,3; Med. 147, I; Protokollbücher der Med. Fakultät Göttingen: Sitzung vom 2. Juli 1933

246 vgl. Univ. Archiv, Kur. 16. III, Ad 103

247 Zimmermann (1997), S. 366-377

vgl. Protokollbücher der Med. Fakultät Göttingen: Sitzungen vom 27. Juli 1939, 26. September 1940, 12. Mai und 21. Oktober 1941, 25. Januar und 25. Februar 1942

250 Die folgenden Aussagen stützen sich auf die Krankenakten der UniversitätsFrauenklinik Göttingen von 1934-1945, die im Archiv Waldweg aufbewahrt werden. vgl. Bock (1986), S. 372

252 Die Aussagen stützen sich auf die Krankenakten der Chirurgischen Universitätsklinik Göttingen von 1935 und 1937-1945 im Archiv Waldweg. vgl. Nieds. Hauptstaatsarchiv Hannover 155 Gö. Acc 58/83 Nr. 4 vgl. Nieds. Hauptstaatsarchiv Hannover 155 Gö. Acc 58/83 Nr. 10 vgl. Suesse/Meyer (1988), S. 114 vgl. Lifton (1988), S. 97 vgl. Nieds. Hauptstaatsarchiv Hannover 155 Gö Acc. 58/83 Nr. 17 vgl. Klee (1986), S. 223

BDC (Berlin Document Center: Akte F. H. Rein); Dokument 959 vgl. Univ. Archiv, Med. 151

Univ. Archiv, Med. 2; (12. Oktober 1945). Stich war der einzige der ordentlichen Professoren in der Liste der "Dismissed members of the medical faculty “, der als emeritiert verzeichnet wurde.

262 Univ. Archiv, Med. 18

263 vgl. Zimmermann (2007), S. 7

264 Cicero, Pro Lucius Mvrena oratio, 42

265 vgl. Steding (2001), S. 160-161

266 vgl. Kirchhoff et al. (2001), S. 155-159

267 vgl. Zimmermann (2001), S. 162-168

268

vgl. Schultz et al. (2001), S. 169-172 



\section{Quellen und Literatur}

\section{Ungedruckte Quellen}

\section{Berlin}

Berlin Document Center (BDC)

\section{Göttingen}

Universitätsarchiv

Sekretariatsakten (Rektoratsakten), Kuratorialakten, Akten, Personalakten und Protokollbücher der Medizinischen Fakultät

Archiv Waldweg

Krankenakten der Universitäts - Frauenklinik Göttingen

Krankenakten der Chirurgischen Universitätsklinik Göttingen

\section{Hannover}

Niedersächsisches Hauptstaatsarchiv

\section{Literatur}

Baldinger, Ernst Gottfried, Das göttingische klinische Institut. Neues Magazin für Aerzte 11 (1789), S. 97-103

Benz, Christoph, Die Geschichte des zahnärztlichen Unterrichts an der Universität Göttingen. Von der ersten Institutsgründung bis zur Gegenwart. Med. Diss. Göttingen 1986

Blaser, Wilhelm, Die Vorgeschichte des zahnärztlichen Doktortitels. Med. Diss. Köln. Greifswald 1937 (Arbeiten der deutsch-nordischen Gesellschaft für Geschichte der Medizin, der Zahnheilkunde und der Naturwissenschaften) 
Blumenbach, Johann Friedrich, Über den Bildungsbetrieb und das Zeugungsgeschäfte. Göttingen 1781 (Nachdruck und mit einem Vorwort und Anmerkungen versehen von László von Károlyi, Stuttgart 1971)

Bock, Gisela, Zwangssterilisation im Nationalsozialismus. Studien zur Rassenpolitik und Frauenpolitik. Opladen 1986

Chronik der Georg-August-Universität zu Göttingen für das Rechungsjahr 1910. Mit Rückblick auf das Jahrzehnt 1901-1910. Göttingen 1911

Cramer, August, Die Heil- und Unterrichtsanstalten für psychische und Nervenkranke in Göttingen. Klin. Jb. 14 (1905), S. 1-40

Cicero, Marcus Tullius, Orationes. Recognovit Brevique Adnotatione Critica Instrvxit, Albertus Cvrtis Clark. Oxford 1961 (Scriptorvm Classicorvm Bibliotheca Oxoniensis)

Deinhard, Johann-Markus, Die Göttinger Sammlung zur Geschichte der Geburtshilfe und Frauenheilkunde mit einem Katalog der geburtshilflichen Sammlung. Med. Diss. Göttingen 1986

Ebel, Wilhelm, (Hrsg.), Die Privilegien und ältesten Statuten der Georg-AugustUniversität zu Göttingen. Göttingen 1961

Ebstein, Erich, Wilhelm Ebsteins Arbeiten aus den Jahren 1859-1906. Dt. Archiv für klinische Medizin 89 (1907), S. 367-378

Ebstein, Erich, Bibliographie der Arbeiten von Wilhelm Ebstein von 1906-1912. Janus 17 (1912), S. IV-X

Ebstein, Wilhelm, Über die Entwicklung des klinischen Unterrichts an der Göttinger Hochschule und über die heutigen Aufgaben der medizinischen Klinik. Klin. Jb. 1 (1889), S. 67-109

Ebstein, Wilhelm, Die Medizinische Klinik. Klin. Jb. 3 (1891), S. 235-246

Eulenburg, Franz, Die Frequenz der deutschen Universitäten von ihrer Gründung bis zur Gegenwart. Leipzig 1904

Eulner, Hans-Heinz, Die Entwicklung der medizinischen Spezialfächer an den Universitäten des deutschen Sprachgebiets. Stuttgart 1970 (Studien zur Medizingeschichte des 19. Jahrhunderts, Bd. IV)

Eulner, Hans-Heinz, Hoepke, Hermann, Der Briefwechsel zwischen Rudolph Wagner und Jacob Henle 1838-1862. Göttingen 1979

Fenner, Jörg, Die Zahnärzte an der Universität Göttingen im 19. Jahrhundert unter besonderer Berücksichtigung des ersten Dozenten für Zahnheilkunde Dr. Johann Heinrich Pauli (1795-1850). Med. Diss. Göttingen 1971

Frensdorff, Ferdinand, (Hrsg.), Ein Bericht über Göttingen, Stadt und Universität aus dem Jahre 1754. Jb. des Geschichtsvereins für Göttingen und Umgebung 1 (1908), S. 43-117 
Frenzel, Hermann, Die Hals-Nasen-Ohren Klinik der Universität Göttingen.

Eine kurze Darstellung ihrer baulichen Entwicklung 1878-1963. Göttingen 1964

Geier, Johannes, Die Geschichte der dermatologischen Universitätsklinik in

Göttingen von ihrer Gründung bis zum Umzug in das jetzige Kliniksgebäude.

Med. Diss. Göttingen 1987

Georg-August-Universität zu Göttingen. Amtliches Namensverzeichnis.

Winterhalbjahr 1932/33

Goethes Werke. Hrsg. im Auftrage der Großherzogin Sophie von Sachsen. II.

Abtheilung, 7. Bd., I. Abtheilung, 35. Bd. Weimar 1892 (Fotomechanischer

Nachdruck München 1987)

Göttinger Tageblatt vom 26. Juli 1933

Gruber, Georg, Zur Geschichte der Psychiatrie in Göttingen. Sudhoffs Archiv 40 (1956), S. 345-371

Hagene, Claudia, Heinrich Martius (1885-1965). Göttinger Gynäkologe und Wissenschaftler. Med. Diss. Göttingen 2006

Haller, Albrecht von, De Partibus Corporis Humani Sensilibus et Irritabilibus. In: Commentarii Societatis Regiae Scientiarum Gottingensis. Tomus II. Ad Annum 1752. Bd. 2. Gottingae 1753, S. 114-158

Haller, Albrecht von, Zürich. GGA (1755), S. 615-616

Haller, Albrecht von, Elementa Physiologiae Corporis Humani. 8 Bde. Lausanne 1757-1766

Hasselhorn, Fritz, Weinreis, Hermann, Göttingens Weg in den Nationalsozialismus, dargestellt anhand der städtischen Wahlergebnisse 1924-1933. In: Stadt Göttingen, Kulturdezernat, (Hrsg.), Göttingen unter dem Hakenkreuz. Nationalsozialistischer Alltag in einer deutschen Stadt. Texte und Materialien, Göttingen 1983, S. 57-67

Haselhorst, Klaus, Johann Wilhelm Heinrich Conradi. Med. Diss. Göttingen 1964

Heischkel, Edith, Medizinischer Unterricht im 17. und 18. Jahrhundert im Spiegel von Briefen und Aufzeichnungen. Dtsch. med. Rundschau 3 (1949), S. 292-294

Henle, Jacob, Pathologische Untersuchungen. Berlin 1840

Henle, Jacob, Allgemeine Anatomie. Lehre von den Mischungs- und Formbestandtheilen des menschlichen Körpers. Leipzig 1841

Henle, Jacob, Handbuch der rationellen Pathologie. Braunschweig 1846-1853

Henle, Jacob, Zur Anatomie der Niere. Göttingen 1862 (Abhandlungen der Königlichen Gesellschaft der Wissenschaften zu Göttingen, Bd. 10)

Henle, Jacob, Handbuch der systematischen Anatomie des Menschen in drei Bänden. 2. Auflage. Braunschweig 1872-1879 
Henoch, Eduard, Über den Unterricht in der Kinderheilkunde. Klin. Jb. 2 (1890), S. 130-139

Himly, Karl Gustav, Göttingen. GGA (1810), S. 529-542

Hintzsche, Erich, Einige kritische Bemerkungen zur Bio- und Ergographie Albrecht von Hallers. Gesnerus 16 (1959), S. 1-15

Hippel, Arthur von, Die neue Universitäts-Augenklinik in Göttingen. Klinische Monatsblätter für Augenheilkunde 45 (1907), S. 217-226

Hollmann, Samuel Christian, Die Georg-Augustus-Universität zu Göttingen, in der Wiege, in Ihrer blühenden Jugend, und reiffererm Alter. Mit unpartheiischer Feder entworfen von Einem Ihrer Ersten, und nun allein noch übrigem, Academischem Lehrer. Göttingen 1787

Kant, Immanuel, Werke in zehn Bänden. Hrsg. von Wilhelm Weischedel. Darmstadt 1968

Kirchhoff, Heinz, Plentz, Ulrike, Schmidt-Jochheim, Anita, Symbole des Weiblichen. Die kulturgeschichtliche Sammlung Heinz Kirchhoff. In: „,Ganz für das Studium angelegt “: Die Museen, Sammlungen und Gärten der Universität Göttingen. Hrsg. von Dietrich Hoffmann und Kathrin Maack-Rheinländer im Auftrage des Universitätsbundes. Göttingen 2001, S. 155-159

Klee, Ernst, „Euthanasie“ im NS-Staat. Frankfurt/Main 1986 (Fischer Tb 4326)

König, Franz, Die Chirurgische Klinik. Klin. Jb. 3 (1891), S. 246-271

Kohlhagen, Werner, Die Pathologische Anatomie in Göttingen während der ersten Hälfte des 19. Jahrhunderts. Göttingen 1935 (Vorarbeiten zur Geschichte der Göttinger Universität und Bibliothek. Hrsg. vom Universitätsbund Göttingen, Heft 18)

Kratsch, Barthel, Das Leben des Geh. Med.-Rathes Wilhelm Ebstein, Ordinarius an der Georg-August-Universität Göttingen. Med. Diss. Göttingen 1989

Krumsteller, Renate, Die Anfänge der medizinischen Poliklinik zu Göttingen. Eine medizin- und kulturhistorische Studie zur zweiten Hälfte des 18. Jahrhunderts, gewonnen aus dem Archivmaterial der Universität. Göttingen 1958 (Arbeiten aus der Staats- und Universitätsbibliothek Göttingen, Hainbergschriften. Neue Folge. Hrsg. von Karl Julius Hartmann, Bd. 3)

Küntzel, Hans, „Aber Fesseln tragen kann ich nicht“ Johannes Brahms und Agathe von Siebold. Göttingen 2003

Kuhn, Hans-Jürgen, siehe Schultz, Michael

Langenbeck, Konrad Johann Martin, Novum Theatrum anatomicum quod Gottingae est a serenissimo ac potentissimo Principe ac Domino Domino Georgio IV. Britanniarum Hannoveraeque Rege conditum d. II. Novembris MDCCCXXIX auguratum. Göttingen 1829 
Lauer, Hans Hugo, Zwei mutige Frauen in ihrer Zeit, Josepha von Siebold und ihre Tochter Charlotte Heidenreich-von Siebold. Medizinischer Monatsspiegel (E. Merck) 2 (1966), S. 27-31

Lichtenberg, Georg Christoph, Schriften und Briefe. Hrsg. von Wolfgang Promies. 4 Bde. München 1967-1992

Lifton, Robert, Ärzte im Dritten Reich. Stuttgart 1988

Luhn, Antke, Geschichte des Frauenstudiums an der Medizinischen Fakultät der Universität Göttingen. Med. Diss. Göttingen 1971

Mantius, Irmgard, Göttingen in der Geschichte der deutschen Augenheilkunde. Med. Diss. Göttingen 1937

Martius, Heinrich, Die Universitäts-Frauenklinik in Göttingen. Von ihrer Gründung im Jahre 1751 als Accouchirhospital am Geissmarthore bis 1931. Leipzig 1931

Marx, Karl Friedrich Heinrich, Goettingen in medicinischer, physischer und historischer Hinsicht geschildert. Versuch einer Topographie von Goettingen. Göttingen 1824

McLaughlin, Peter, Blumenbach und der Bildungstrieb. Zum Verhältnis von epigenetischer Embryologie und typologischem Artbegriff. Med. Hist. Journal 17 (1982), S. 357-372

Merkel, Friedrich, Jacob Henle. Ein deutsches Gelehrtenleben nach Aufzeichnungen und Erinnerungen erzählt. Braunschweig 1891

Meyer, Ludwig, Die psychiatrische Klinik der königlichen Georg-August-Universität in Göttingen. Klin. Jb. 1 (1889), S. 212-217

Orth, Johannes, Das pathologische Institut. Klin. Jb. 3 (1891), S. 271-278

Osiander, Friedrich Benjamin, Denkwürdigkeiten für die Heilkunde und Geburtshülfe aus den Tagebüchern der königlichen practischen Anstalten zu Erlernung dieser Wissenschaften in Göttingen ausgehoben. 2 Bde. Göttingen 1794

Plentz, Ulrike, siehe Kirchhoff, Heinz

Pütter, Johann Stephan, Versuch einer academischen Gelehrten=Geschichte von der Georg-August-Universität zu Göttingen. 3 Bde. Göttingen 1763-1820

Radtke, Joachim, Geschichte der Kinderheilkunde an der Universität Göttingen. Med. Diss. Göttingen 1974

Reckow, Joachim von, Grundlagen zur Geschichte der deutschen zahnärztlichen Approbation bis 1913. Greifswald 1927 (Arbeiten der deutsch-nordischen Gesellschaft für Geschichte der Medizin, der Zahnheilkunde und der Naturwissenschaften, Bd. 4)

Richter, August Gottlieb, Göttingen. GGA (1781), S. 376 
Richter, August Gottlieb, Medizinische und chirurgische Bemerkungen, vorzüglich im öffentlichen akademischen Hospitale gesammlet. Göttingen 1793

Richter, Gottfried, Das anatomische Theater. Berlin 1936

Richter, Wolfgang, Die Geschichte und Entwicklung der Hals-Nasen-Ohrenheilkunde in Göttingen von 1737 bis 1963. Med. Diss. Göttingen 1986

Rieberer, Gabriela, Das Institut für Medizinische Chemie und Hygiene der Universität Göttingen von der Gründung 1883 bis 1955. Med. Diss. Göttingen 1990

Rössler, Emil Franz, Die Gründung der Universität Göttingen. Göttingen 1855

Schmidt-Jochheim, Anita, siehe Kirchhoff, Heinz

Schultz, Michael, Kuhn, Hans-Jürgen, Die Blumenbachsche Schädelsammlung in der Göttinger Anatomie. In: „Ganz für das Studium angelegt“: Die Museen, Sammlungen und Gärten der Universität Göttingen. Hrsg. von Dietrich Hoffmann und Kathrin Maack-Rheinländer im Auftrage des Universitätsbundes. Göttingen 2001, S. 169-172

Selle, Götz von, Die Georg-August-Universität zu Göttingen. Göttingen, 1937

Selle, Götz von, Universität Göttingen. Wesen und Geschichte. Göttingen 1953

Siebold, Eduard Karl Jacob, Versuch einer Geschichte der Geburtshilfe. 2 Bde. Berlin 1839-1845

Sprengel, Kurt, Versuch einer pragmatischen Geschichte der Arzneykunde. 5 Bde. Halle 1800-1803

Steding, Gerd, Die Humanembryologische Dokumentationssammlung Blechschmidt der Abteilung Embryologie des Zentrums Anatomie. In: „Ganz für das Studium angelegt‘: Die Museen, Sammlungen und Gärten der Universität Göttingen. Hrsg. von Dietrich Hoffmann und Kathrin Maack-Rheinländer im Auftrage des Universitätsbundes. Göttingen 2001, S. 160-161

Sueße, Thorsten, Meyer Heinrich, Abtransport der „Lebensunwerten“. Die Konfrontation niedersächsischer Anstalten mit der NS-,„Euthanasie“. Hannover 1988

Thode, Brita, Die Göttinger Anatomie 1733-1828. Med. Diss. Göttingen 1979

Welter, Hela, Die Geschichte der Dermatologie an der Universität Göttingen (17371934). Göttingen 1935 (Vorarbeiten zur Geschichte der Göttinger Universität und Bibliothek. Hrsg. vom Universitätsbund Göttingen, Heft 17)

Winkelmann, Heike, Das akademische Hospital in Göttingen von 1781 bis 1850. Zur Geschichte der Göttinger Universitätsklinik. Med. Diss. Göttingen 1981

Winkelmann, Heike, Entbindungswissenschaft und Entbindungskunst bei Friedrich Benjamin Osiander. Med. Hist. Journal 18 (1983), S. 306-312

Zimmermann, Johann Georg, Das Leben des Herrn Haller. Zürich 1755 
Zimmermann, Volker, Karl Saller und die Einrichtung eines »Lehrstuhl für Rassenhygiene« an der Georg-August-Universität Göttingen.

In: Medizingeschichte und Gesellschaftskritik. Festschrift für Gerhard Baader.

Hrsg. von Michael Hubenstorf et al.. Husum 1997, S. 366-379 (Abhandlungen zur Geschichte der Medizin und der Naturwissenschaften, Heft 81)

Zimmermann, Volker, „Metalla ferri. optumo pessimoque vitae instrumento“. Die

Sammlung zur Geschichte der Geburtshilfe. In: „Ganz für das Studium angelegt“:

Die Museen, Sammlungen und Gärten der Universität Göttingen. Hrsg. von

Dietrich Hoffmann und Kathrin Maack-Rheinländer im Auftrage des

Universitätsbundes. Göttingen 2001, S. 162-168

Zimmermann, Volker, Richter, August Gottlieb. In: Enzyklopädie der

Medizingeschichte. Hrsg. von Werner E. Gerabek et al.. Berlin 2005, S. 1250

Zimmermann, Volker, (Hrsg.), Leiden verwehrt Vergessen, Zwangsarbeiter in

Göttingen und ihre medizinische Versorgung in den Universitätskliniken.

Göttingen 2007 



\section{Bildnachweise}

Mit Ausnahme von Nr. 7, 13, 32, 38, 39, 40, 41 hat Ronald Schmidt sämtliche Abbildungen erstellt. Die jeweiligen Vorlagen sind entsprechend aufgeführt.

Hülsken, Werner (Göttingen), Medizinische Fakultät, Presseabteilung: Abb. 40, 41 Institut für Ethik und Geschichte der Medizin (Göttingen): Abb. 6, 9, 19, 20, 22, 29, 30

Schmidt, Ronald (Göttingen): Abb. 11, 14, 16, 17, 18, 21, 24, 25, 26, 27, 31, 33, 34, $35,36,37$

Schultz, Michael (Göttingen): Abb. 38, 39

Städtisches Museum (Göttingen): Abb. 1, 2, 3, 4, 5, 8, 10, 12, 15, 23, 28

Universitätsbibliothek (Göttingen), Handschriftenabteilung: Abb. 32

Weiß, Alexander (Göttingen): Abb. 7, 13 

ie Gründung der Georg-August-Universität Göttingen beruhte auf einem weitgehend neuen Konzept, das vom Geist der Aufklärung durchzogen auf Toleranz gründete und jeglichem Extremismus und Sekretierertum abhold war. Diesem Prinzip war auch die Medizinische Fakultät von Anfang an verpflichtet.

Durch das von ihr vertretene Ausbildungsmodell des praktisch-klinischen Unterrichts am Krankenbett gelang es bereits in der Aufbauphase entscheidende Impulse zu setzen, die für die medizinische Ausbildung über Göttingen hinaus richtungsweisend wurden. Damit trug die Medizinische Fakultät wesentlich dazu bei, den internationalen Ruhm der Georgia-Augusta zu begründen.

Der Abriss mit seiner vielschichtigen Realität vermittelt Einsichten in das Selbstverständnis, die Selbstwahrnehmung und die Selbstdarstellung der Medizinischen Fakultät in ihrer Geschichte.

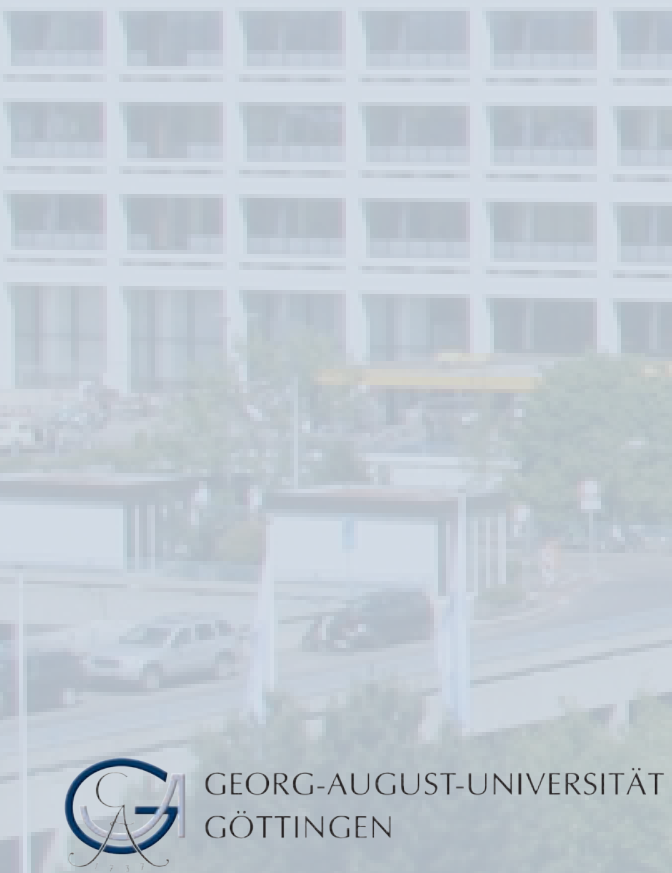

